\title{
Readily Tunable and Bifunctional $L$-Prolinamide Derivatives: Design and Application in the Direct Enantioselective Aldol Reactions
}

\author{
Jia-Rong Chen, Hai-Hua Lu, Xin-Yong Li, Lin Chen, Jian Wan \\ and Wen-Jing Xiao* \\ The Key Laboratory of Pesticide \& Chemical Biology, Ministry of Education; College of Chemistry, Central China \\ Normal University, 152 Luoyu Road, Wuhan, Hubei, 430079, China \\ wxiao@mail.ccnu.edu.cn
}

Supporting Information

\section{Table of Contents}

General information.................................................. 2

General procedure for the synthesis of catalysts $1 \mathrm{a}-\mathrm{e}$ and $1 \mathrm{f}$ and data..........S2-5

General procedure for the Aldol reaction and data of $4 \mathrm{a}-\mathrm{k}$ and $6 \ldots \ldots \ldots \ldots . . . .55-8$

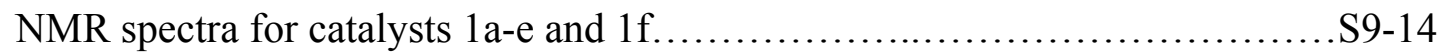

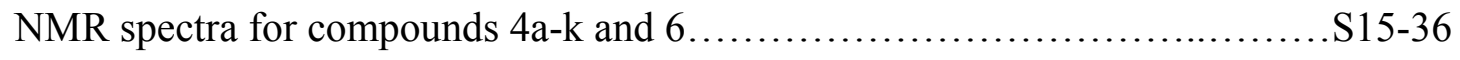

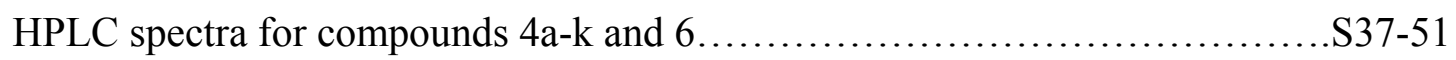

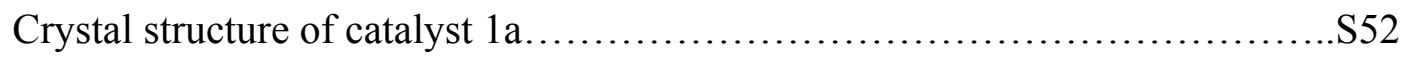

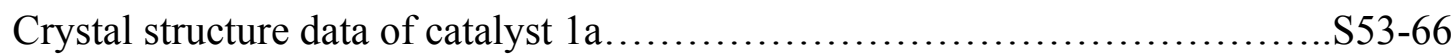




\section{Experimental Section}

General Information: Unless otherwise noted, material were purchased from commercial suppliers and used without further purification. Dichloromethane and trichloromethane were freshly distilled from calcium hydride. Hexane and ethyl acetate for flash column chromatography were distilled before use. Flash column chromatography was performed using 200-300 mesh silica gel. Infrared spectra were recorded on a Perkin-Elmer PE-983 spectrometer as $\mathrm{KBr}$ film with absorption in $\mathrm{cm}^{-1} .{ }^{1} \mathrm{HNMR}$ spectra were recorded on Varian Mercury 400 (400 MHz) spectrophotometers. Chemical shifts are reported in ppm from the solvent resonance as the internal standard $\left(\mathrm{CDCl}_{3}: 7.26 \mathrm{ppm}\right)$. Data are reported as follows: chemical shift, multiplicity $(\mathrm{s}=$ single, $\mathrm{d}=\mathrm{doublet}, \mathrm{t}=$ triplet, $\mathrm{q}=$ quartet, $\mathrm{br}=$ broad, $\mathrm{m}=$ multiplet $)$, coupling constants $(\mathrm{Hz})$ and integration. ${ }^{13} \mathrm{CNMR}$ spectra were recorded on Varian Mercury $400(100 \mathrm{MHz})$ with complete proton decoupling spectrophotometers $\left(\mathrm{CDCl}_{3}: 77.0\right.$ ppm). Chiral HPLC was performed on Agilent 1100 series with chiral columns (Chirapak AS, AD, OD and OJ columns, (Daicel Chemical Ind., Ltd.)). Elementary analysis was taken on a Vario EL III elementary analysis instrument. Mass spectra analysis was performed on API 200 LC/MS system (Applied Biosystems Co. Ltd.). Optical rotations were measured on a JASCO P-1010 Polarimeter at $\lambda=589 \mathrm{~nm}$.

\section{General procedure for the synthesis of catalysts 1a-e and 1f}

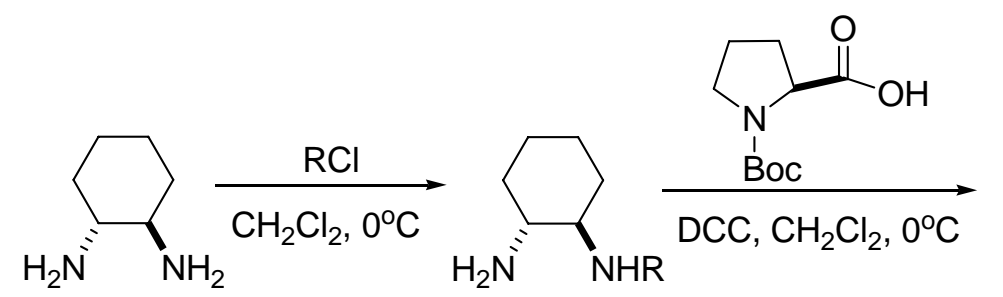

$(1 \mathrm{R}, 2 \mathrm{R})$

C-1

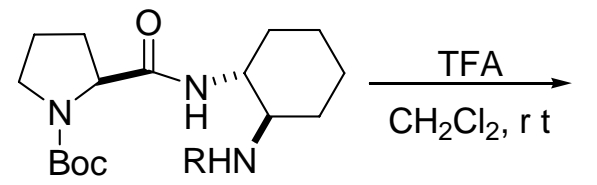

C-2

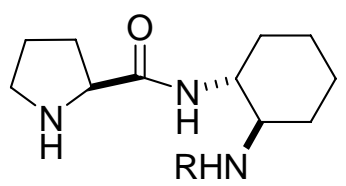

1a-e or $1 f$

C-3

\section{Typical procedure for the synthesis of C-1}

$(1 \mathrm{R}, 2 \mathrm{R})$-1, 2-diaminocyclohexane $(3.42 \mathrm{~g}, 30 \mathrm{mmol})$ was added to dichloromethane. The mixture was cooled down to $0^{\circ} \mathrm{C}$ and a solution of acetyl chloride $(10 \mathrm{~mol})$ in $10 \mathrm{~mL}$ of dichloromethane was added dropwise over 30 min. After the addition was complete, the mixture was allowed to warm to room temperature and stirred overnight. The resulting solution was washed with water and the solvent removed at reduced pressure. The residue was purified through column chromatography on silica gel (eluent, ethyl acetate / methanol 2:1) to give C-1.

\section{Typical procedure for the synthesis of $\mathrm{C}-2$}

N-t-butyloxycarbonyl-L-Proline (4.3 g, $20 \mathrm{mmol}$ ) and N, N'-dicyclohexylcarbodiimide (DCC) (4.15 g, $20 \mathrm{mmol})$ were dissolved in $40 \mathrm{~mL}$ dichloromethane and cooled down to $0^{\circ} \mathrm{C}$. After the solution was stirred for $30 \mathrm{~min}$, a solution of C-1 (16 mmol) in $20 \mathrm{~mL}$ dichloromethane was added dropwise over $15 \mathrm{~min}$. After the addition was complete, the mixture was warmed to room temperature and stirred for another $12 \mathrm{~h}$. After filtration and removal of solvent at reduced pressure, the residue was purified through flash column chromatography on silica gel (eluent, hexane / ethyl acetate $2: 1$ ) to provide C-2. 


\section{Typical procedure for the synthesis of C-3}

C-2 (3.26 g, $8 \mathrm{mmol})$ was dissolved in mixture of TFA/ $\mathrm{CH}_{2} \mathrm{Cl}_{2}(1: 4)(20 \mathrm{~mL})$, and stirred for $2 \mathrm{~h}$ at room temperature. The mixture was basified with concentrated ammonia solution and extracted with dichloromethane $(3 \times 30 \mathrm{~mL})$. After the removal of the solvent at reduced pressure, the residue was purified through flash column chromatography on silica gel (eluent, ethyl acetate / methanol 5:1) to yield 1a-e or 1f.

(2S, 1'R, 2'R)-Pyrrolidine-2-carboxylic acid [2'-(4-methylphenylsulfonamido)-cyclohexyl]-amide (1a): White solid: mp: $174.9-175.9{ }^{\circ} \mathrm{C} ;[\alpha]^{29}=+10.2\left(\mathrm{c}=0.54, \mathrm{CHCl}_{3}\right) ;{ }^{1} \mathrm{HNMR}\left(400 \mathrm{MHz}, \mathrm{CDCl}_{3}\right) \delta(\mathrm{ppm})$ 1.09-1.27 (m, 4H), 1.57-2.13 (m, 8H), $2.38(\mathrm{~s}, 3 \mathrm{H}), 3.02-3.08(\mathrm{~m}, 1 \mathrm{H}), 3.55-3.62(\mathrm{~m}, 1 \mathrm{H}), 3.70-3.73(\mathrm{~m}, 1 \mathrm{H}), 7.24(\mathrm{~d}, J=8.4 \mathrm{~Hz}, 2 \mathrm{H})$, $7.71(\mathrm{~d}, J=8.0 \mathrm{~Hz}, 2 \mathrm{H}), 7.81(\mathrm{~d}, J=8.4 \mathrm{~Hz}, 1 \mathrm{H}) ;{ }^{13} \mathrm{CNMR}\left(100 \mathrm{MHz}, \mathrm{CDCl}_{3}\right) \delta(\mathrm{ppm}) 21.4,24.5,26.2,30.6,32.3$, 33.3, 47.1, 51.8, 58.6, 60.3, 126.6, 129.4, 139.3, 142.7, 176.4; IR (KBr) 3288, 3128, 3026, 2954, 2869, 1637, 1531, 1452, 1326, 1164, 1047, 812, $729 \mathrm{~cm}^{-1}$; MS (ESI) Calcd. for $\mathrm{MNa}^{+} 388$, found 388; Anal. Calcd. For $\mathrm{C}_{18} \mathrm{H}_{27} \mathrm{~N}_{3} \mathrm{O}_{3} \mathrm{~S}$ : C, 59.15; H, 7.45; N, 11.54. Found: C, 59.15; H, 7.53; N, 11.72.

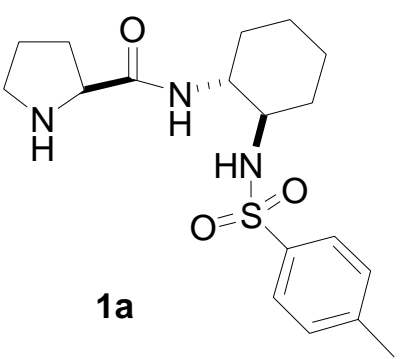

(2S, 1'R, 2'R)-Pyrrolidine-2-carboxylic acid [2'-(4-methylbenzamido)-cyclohexyl]-amide (1b): White solid; mp: 189.6-191.2 ${ }^{\circ} \mathrm{C} ;[\alpha]^{29}{ }_{\mathrm{D}}=-69.6\left(\mathrm{c}=0.51, \mathrm{CHCl}_{3}\right) ;{ }^{1} \mathrm{HNMR}\left(400 \mathrm{MHz}, \mathrm{CDCl}_{3}\right) \delta(\mathrm{ppm})$ 1.24-1.63 (m, 7H), 1.75-2.24 (m, 5H), 2.37 (s, 3H), 2.78-2.95 (m, 2H), 3.72-3.82 (m, 3H), $7.04(\mathrm{~d}, J=5.2 \mathrm{~Hz}, 1 \mathrm{H}), 7.19(\mathrm{~d}, J=8.0 \mathrm{~Hz}$, $2 \mathrm{H}), 7.68(\mathrm{~d}, J=8.4 \mathrm{~Hz}, 2 \mathrm{H}), 7.89(\mathrm{~s}, 1 \mathrm{H}) ;{ }^{13} \mathrm{CNMR}\left(100 \mathrm{MHz}, \mathrm{CDCl}_{3}\right) \delta(\mathrm{ppm}) 21.2,24.5,24.8,25.5,30.6,31.8$, 32.2, 46.8, 51.8, 55.3, 60.1, 126.9, 128.9, 131.1, 141.4, 167.0, 175.6; IR (KBr) 3332, 2933, 2857, 1629, 1516, 1449, 1327, 1162, 1044, 833, $756 \mathrm{~cm}^{-1}$; MS (ESI) Calcd. for $\mathrm{MNa}^{+} 352$, found 352; Anal. Calcd. For $\mathrm{C}_{19} \mathrm{H}_{27} \mathrm{~N}_{3} \mathrm{O}_{2}$ : C, 69.27; H, 8.26; N, 12.76. Found: C, 69.29; H, 8.20; N, 12.67.

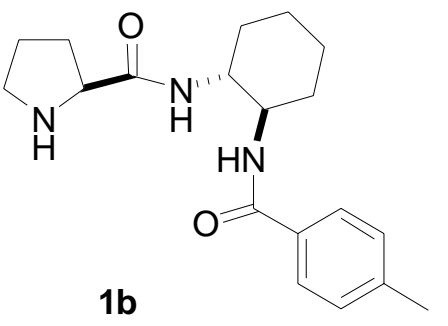

(2S, 1'R, 2'R)-Pyrrolidine-2-carboxylic acid [2'-(4-chlorolbenzamido)-cyclohexyl]-amide (1c): White solid; mp: $180.2-183.7{ }^{\circ} \mathrm{C} ;[\alpha]^{29}{ }_{\mathrm{D}}=-65.4\left(\mathrm{c}=0.53, \mathrm{CHCl}_{3}\right) ;{ }^{1} \mathrm{HNMR}\left(400 \mathrm{MHz}, \mathrm{CDCl}_{3}\right) \delta(\mathrm{ppm})$ 1.24-2.27 (m, 13H) 2.76-2.94 (m, 2H), 3.69-3.80 (m, 3H), 7.29 (d, $J=4.8 \mathrm{~Hz}, 1 \mathrm{H}), 7.37$ (d, $J=8.4 \mathrm{~Hz}, 2 \mathrm{H}), 7.76(\mathrm{~d}, J=8.0 \mathrm{~Hz}, 2 \mathrm{H})$, $7.85(\mathrm{~d}, J=6.8 \mathrm{~Hz}, 1 \mathrm{H}) ;{ }^{13} \mathrm{CNMR}\left(100 \mathrm{MHz}, \mathrm{CDCl}_{3}\right) \delta(\mathrm{ppm}) 24.5,24.7,25.3,3.5,31.7,32.0,46.6,52.4,55.2$, 60.0, 128.4, 128.5, 132.4, 137.4, 166.4, 174.4; IR (KBr) 3330, 2936, 2858, 1674, 1632, 1516, 1203, 1140, 1095, 843, $759 \mathrm{~cm}^{-1}$; MS (ESI) Calcd. for $\mathrm{MNa}^{+} 372$, found 372; Anal. Calcd. For $\mathrm{C}_{18} \mathrm{H}_{24} \mathrm{ClN}_{3} \mathrm{O}_{2}$ : C, 61.79; H, 6.91; N, 12.01. Found: C, 61.68; H, 6.82; N, 12.14 . 


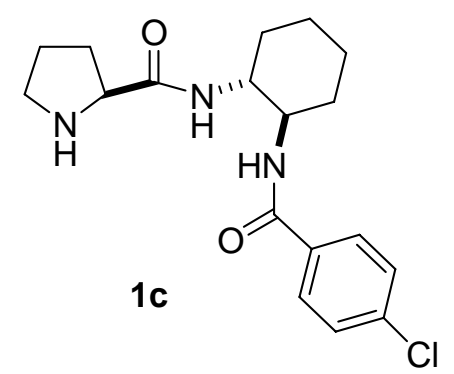

(2S, 1'R, 2'R)-Pyrrolidine-2-carboxylic acid [2'-(2,2,2-trifluoroacetamido)cyclohexyl]-amide (1d): White solid. mp: $180.2-183.7{ }^{\circ} \mathrm{C} ;[\alpha]^{29}{ }_{\mathrm{D}}=+7.8\left(\mathrm{c}=0.58, \mathrm{CHCl}_{3}\right) ;{ }^{1} \mathrm{HNMR}\left(400 \mathrm{MHz}, \mathrm{CDCl}_{3}\right) \delta(\mathrm{ppm}) 1.29-1.38(\mathrm{~m}, 4 \mathrm{H})$, 1.57-2.18 (m, 9H), 2.79-2.99 (m, 2H), 3.56-3.78 (m, 3H), $7.82(\mathrm{~d}, J=8.4 \mathrm{~Hz}, 1 \mathrm{H}), 7.89(\mathrm{~d}, J=5.2 \mathrm{~Hz}, 1 \mathrm{H})$; ${ }^{13} \mathrm{CNMR}\left(100 \mathrm{MHz}, \mathrm{CDCl}_{3}\right) \delta$ (ppm) 24.1, 24.7, 25.9, 30.8, 31.3, 31.7, 47.1, 51.1, 56.5, 60.2, 111.5, 114.4, 117.2, 12.1; IR (KBr) 3235, 2936, 2863, 1709, 1656, 1528, 1194, 1149, 1102, $722 \mathrm{~cm}^{-1}$; MS (ESI) Calcd. for $\mathrm{MNa}^{+} 330$, found 330; Anal. Calcd. For $\mathrm{C}_{13} \mathrm{H}_{20} \mathrm{~F}_{3} \mathrm{~N}_{3} \mathrm{O}_{2}$ : C, 50.81; H, 6.56; N, 13.67. Found: C, 50.48; H, 6.56; N, 13.47.<smiles>O=C(NC1CCCCC1NC(=O)C(F)(F)F)N1CCCC1</smiles>

(2S, 1'R, 2'R)-Pyrrolidine-2-carboxylic acid [2'-(2-trifluoroacetamido)cyclohexyl]-amide (1e): White solid. mp: $158.6-159.9{ }^{\circ} \mathrm{C} ;[\alpha]^{29}{ }_{\mathrm{D}}=+14.1\left(\mathrm{c}=0.50, \mathrm{CHCl}_{3}\right) ;{ }^{1} \mathrm{HNMR}\left(400 \mathrm{MHz}, \mathrm{CDCl}_{3}\right) \delta(\mathrm{ppm}) 1.12-1.22(\mathrm{~m}, 4 \mathrm{H})$, 1.46-2.02 (m, 8H), $2.32(\mathrm{~s}, 1 \mathrm{H}), 2.73-2.89(\mathrm{~m}, 2 \mathrm{H}) 3.58-3.64(\mathrm{~m}, 3 \mathrm{H}), 4.45-4.70(\mathrm{~m}, 2 \mathrm{H}), 6.92(\mathrm{~d}, J=5.2 \mathrm{~Hz}, 1 \mathrm{H})$, $7.64(\mathrm{~d}, J=7.6 \mathrm{~Hz}, 1 \mathrm{H}) ;{ }^{13} \mathrm{CNMR}\left(100 \mathrm{MHz}, \mathrm{CDCl}_{3}\right) \delta(\mathrm{ppm})$ 24.3, 24.5, 25.6, 30.8, 31.8, 31.9, 46.9, 51.3, 53.9, 60.1, 78.7, 80.6, 167.2, 167.4, 175.8; IR (KBr) 3302, 3272, 3079, 2929, 2858, 1645, 1544, 1040, $754 \mathrm{~cm}^{-1}$; MS (ESI) Calcd. for $\mathrm{MNa}^{+}$294, found 294; Anal. Calcd. For $\mathrm{C}_{13} \mathrm{H}_{22} \mathrm{FN}_{3} \mathrm{O}_{2}: \mathrm{C}, 57.55 ; \mathrm{H}, 8.17 ; \mathrm{N}$, 15.49. Found: C, 57.66; H, 8.08; N, 15.38 .

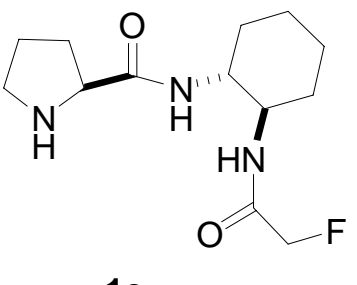

$1 e$

(2S, 1'S, 2'S)- pyrrolidine-2-carboxylic acid [2'-(4-methylbenzamido)-cyclohexyl]-amide (1f): White solid. mp: $219.7-220.6{ }^{\circ} \mathrm{C} ;[\alpha]^{29}{ }_{\mathrm{D}}=-1.9\left(\mathrm{c}=0.52, \mathrm{CHCl}_{3}\right) ;{ }^{1} \mathrm{HNMR}\left(400 \mathrm{MHz}, \mathrm{CDCl}_{3}\right) \delta(\mathrm{ppm})$ 1.26-1.37 (m, 4H), 1.56-2.2 (m, 8H), $2.29(\mathrm{~s}, 3 \mathrm{H}), 2.79-2.88(\mathrm{~m}, 2 \mathrm{H}), 3.49-3.51(\mathrm{~m}, 1 \mathrm{H}), 3.73-3.75(\mathrm{~m}, 2 \mathrm{H}), 7.11(\mathrm{~d}, J=7.6 \mathrm{~Hz}, 2 \mathrm{H})$, $7.18(\mathrm{~s}, 1 \mathrm{H}), 7.62(\mathrm{~d}, J=8.4 \mathrm{~Hz}, 2 \mathrm{H}), 7.73(\mathrm{~d}, J=7.6 \mathrm{~Hz}, 1 \mathrm{H}) ;{ }^{13} \mathrm{CNMR}\left(100 \mathrm{MHz}, \mathrm{CDCl}_{3}\right) \delta(\mathrm{ppm}) 21.3,24.4$, 24.9, 25.9, 30.5, 32.1, 32.2, 47.0, 51.8, 55.7, 30.1, 126.9, 129.0, 131.5, 141.3, 167.1, 176.3; IR (KBr) 3293, 2925, 2856, 1649, 1630, 1542, 1443, 1334, 1116, 832, $679 \mathrm{~cm}^{-1}$; MS (ESI) Calcd. for $\mathrm{MNa}^{+} 352$, found 352; Anal. Calcd. For $\mathrm{C}_{19} \mathrm{H}_{27} \mathrm{~N}_{3} \mathrm{O}_{2}$ : C, 69.27; H, 8.26; N, 12.76. Found: C, 69.06; H, 8.09; N, 12.66. 


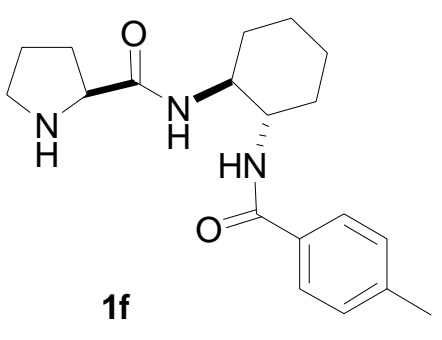

\section{General procedure for the Aldol reaction}

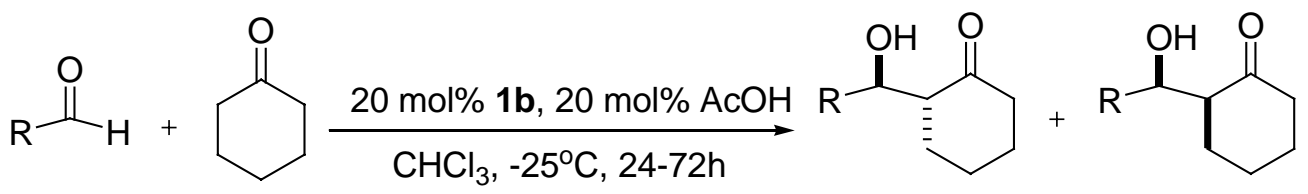

The L-Prolinamide derivatives $1 \mathrm{~b}(0.1 \mathrm{mmol})$ was stirred in $2 \mathrm{~mL}$ of trichloromethane/cyclohexanone (1:1) for 20 min at $-25{ }^{\circ} \mathrm{C}$. The corresponding aldehyde $(0.5 \mathrm{mmol})$ was added and the mixture was stirred for $48-72 \mathrm{~h}$. The mixture was treated with $10 \mathrm{~mL}$ of saturated ammonium chloride solution and extracted with ethyl acetate. The organic layer was dried $\left(\mathrm{MgSO}_{4}\right)$, filtered and concentrated to give pure Aldol adduct through flash column chromatography on silica gel (hexane / ethyl acetate (3:1)).

2-[Hydroxy-(4-nitro-phenyl)-methyl]-cyclohexanone (4a): Reaction time: $72 \mathrm{~h}$; yield: 81\%; Anti/Syn= 98/2, anti-diastereomer, ${ }^{1} \mathrm{HNMR}\left(400 \mathrm{MHz}, \mathrm{CDCl}_{3}\right) \delta(\mathrm{ppm})$ 1.29-2.09 (m, 6H), 2.30-2.47 (m, 2H), 2.55-2.61 (m, 1H), $4.10(\mathrm{~s}, 1 \mathrm{H}), 4.87(\mathrm{~d}, J=8.4 \mathrm{~Hz}, 1 \mathrm{H}), 7.48(\mathrm{t}, J=8.0 \mathrm{~Hz}, 1 \mathrm{H}), 8.16(\mathrm{~d}, J=8.4 \mathrm{~Hz}, 2 \mathrm{H}) ;{ }^{13} \mathrm{CNMR}(100 \mathrm{MHz}$, $\left.\mathrm{CDCl}_{3}\right) \delta(\mathrm{ppm}) 24.5,27.5,30.6,42.5,57.0,73.8,123.4,127.8,147.4,148.4,214.6$; HPLC analysis Chiralcel $\mathrm{AD}-\mathrm{H}$ (Hexane/ $\mathrm{i}-\mathrm{PrOH}=95 / 5,1.0 \mathrm{~mL} / \mathrm{min}, 254 \mathrm{~nm}, 2{ }^{\circ} \mathrm{C}$ ) $\mathrm{t}_{R}$ (minor) $50.2 \mathrm{~min}$ and $\mathrm{t}_{R}$ (major) $67.7 \mathrm{~min}$, ee: 97\%; syn-diastereomer, ${ }^{1} \mathrm{HNMR}\left(400 \mathrm{MHz}, \mathrm{CDCl}_{3}\right) \delta(\mathrm{ppm})$ 1.50-2.13 (m, 6H), 2.39-2.50 (m, 2H), 2.61-2.65 (m, 1H), $3.20(\mathrm{~s}, 1 \mathrm{H}), 5.48(\mathrm{~d}, J=2.0 \mathrm{~Hz}, 1 \mathrm{H}), 7.48(\mathrm{~d}, J=8.8 \mathrm{~Hz}, 2 \mathrm{H}), 8.20(\mathrm{~d}, J=8.8 \mathrm{~Hz}, 2 \mathrm{H}) ;{ }^{13} \mathrm{CNMR}(100 \mathrm{MHz}$, $\left.\mathrm{CDCl}_{3}\right) \delta(\mathrm{ppm}) 24.7,25.9,27.8,42.6,56.7,70.1,123.4,126.6,147.0,149.1,214.1$. HPLC analysis Chiralcel $\mathrm{AD}-\mathrm{H}\left(\right.$ Hexane $\left./ \mathrm{i}-\mathrm{PrOH}=95 / 5,1.0 \mathrm{~mL} / \mathrm{min}, 254 \mathrm{~nm}, 20^{\circ} \mathrm{C}\right) \mathrm{t}_{R} 35.4 \mathrm{~min}$ and $\mathrm{t}_{R} 45.5 \mathrm{~min}$;

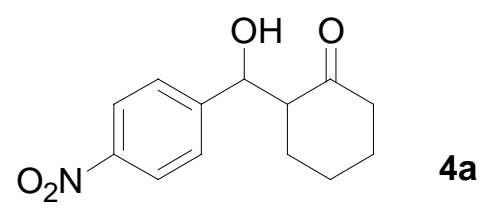

2-[Hydroxy-(2-nitro-phenyl)-methyl]-cyclohexanone (4b): Reaction time: $21 \mathrm{~h}$; yield: 94\%; Anti/Syn= 99/1, anti-diastereomer, ${ }^{1} \mathrm{HNMR}\left(400 \mathrm{MHz}, \mathrm{CDCl}_{3}\right) \delta(\mathrm{ppm})$ 1.51-1.78 (m, 6H), 2.25-2.40 (m, 2H), 2.69-2.75 (m, 1H), 4.04 (s, 1H), 5.39 (d, $J=7.2 \mathrm{~Hz}, 1 \mathrm{H}), 7.37$ (t, $J=8.0 \mathrm{~Hz}, 1 \mathrm{H}), 7.57$ (t, $J=7.6 \mathrm{~Hz}, 1 \mathrm{H}), 7.70$ (d, $J=8.0 \mathrm{~Hz}, 1 \mathrm{H})$, $7.76(\mathrm{~d}, J=8.0 \mathrm{~Hz}, 1 \mathrm{H}) ;{ }^{13} \mathrm{CNMR}\left(100 \mathrm{MHz}, \mathrm{CDCl}_{3}\right) \delta(\mathrm{ppm})$ 24.7, 27.6, 30.8, 42.5, 57.1, 69.3, 123.8, 128.2, 128.9, 132.9, 136.3, 148.6, 214.5; HPLC analysis Chiralcel OD-H (Hexane/ i-PrOH = 95/5, $1.0 \mathrm{~mL} / \mathrm{min}, 254 \mathrm{~nm}$, $20{ }^{\circ} \mathrm{C}$ ) $\mathrm{t}_{R}$ (major) $38.8 \mathrm{~min}$ and $\mathrm{t}_{R}$ (minor) $47.2 \mathrm{~min}$, ee: 96\%; syn-diastereomer, ${ }^{1} \mathrm{HNMR}\left(400 \mathrm{MHz}, \mathrm{CDCl}_{3}\right) \delta$ (ppm) $1.47-2.08(\mathrm{~m}, 6 \mathrm{H}), 2.33-2.43(\mathrm{~m}, 2 \mathrm{H}), 2.80-2.84(\mathrm{~m}, 1 \mathrm{H}), 3.70(\mathrm{~s}, 1 \mathrm{H}), 5.92(\mathrm{~d}, J=2.0 \mathrm{~Hz}, 1 \mathrm{H}), 7.38(\mathrm{t}, J=$ $8.4 \mathrm{~Hz}, 1 \mathrm{H}), 7.60$ (t, $J=7.2 \mathrm{~Hz}, 1 \mathrm{H}), 7.80(\mathrm{~d}, J=7.6 \mathrm{~Hz}, 1 \mathrm{H}), 7.94(\mathrm{~d}, J=8.4 \mathrm{~Hz}, 1 \mathrm{H}) ;{ }^{13} \mathrm{CNMR}(100 \mathrm{MHz}$, $\left.\mathrm{CDCl}_{3}\right) \delta(\mathrm{ppm})$ 24.7, 26.3, 27.2, 42.3, 54.8, 66.3, 124.5, 127.8, 129.5, 133.0, 137.1, 213.7; HPLC analysis Chiralcel OD-H (Hexane/ i-PrOH $\left.=95 / 5,1.0 \mathrm{~mL} / \mathrm{min}, 254 \mathrm{~nm}, 20{ }^{\circ} \mathrm{C}\right) \mathrm{t}_{R} 24.0 \mathrm{~min}$ and $\mathrm{t}_{R} 26.9 \mathrm{~min}$.

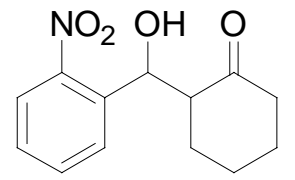


2-[Hydroxy-(3-nitro-phenyl)-methyl]-cyclohexanone (4c): Reaction time: $21 \mathrm{~h}$; yield: 78\%; Anti/Syn= 98/2, anti-diastereomer, ${ }^{1} \mathrm{HNMR}\left(400 \mathrm{MHz}, \mathrm{CDCl}_{3}\right) \delta(\mathrm{ppm})$ 1.33-2.10 (m, 6H), 2.32-2.48 (m, 2H), 2.58-2.64 (m, 1H), 4.14 (s, 1H), 4.87 (d, $J=8.4 \mathrm{~Hz}, 1 \mathrm{H}), 7.50$ (t, $J=8.0 \mathrm{~Hz}, 1 \mathrm{H}), 7.64$ (t, $J=7.6 \mathrm{~Hz}, 1 \mathrm{H}), 8.12(\mathrm{~d}, J=7.6 \mathrm{~Hz}, 1 \mathrm{H})$, $8.18(\mathrm{~d}, \mathrm{~J}=1.6 \mathrm{~Hz}, 1 \mathrm{H}) ;{ }^{13} \mathrm{CNMR}\left(100 \mathrm{MHz}, \mathrm{CDCl}_{3}\right) \delta(\mathrm{ppm}) 24.5,27.5,30.6,42.5,57.0,73.8,121.9,122.7$, 129.2, 133.1, 143.2, 148.1, 214.7; HPLC analysis Chiralcel AD-H (Hexane/ i-PrOH = 95/5, $1.0 \mathrm{~mL} / \mathrm{min}, 254 \mathrm{~nm}$, $\left.20{ }^{\circ} \mathrm{C}\right) \mathrm{t}_{R}$ (major) $38.2 \mathrm{~min}$ and $\mathrm{t}_{R}$ (minor) $50.0 \mathrm{~min}$, ee: 97\%; syn-diastereomer, ${ }^{1} \mathrm{HNMR}\left(400 \mathrm{MHz}, \mathrm{CDCl}_{3}\right) \delta$ (ppm) 1.48-2.10 (m, 6H), 2.33-2.46 (m, 2H), 2.62-2.66 (m, 1H), $3.27(\mathrm{~s}, 1 \mathrm{H}), 5.44(\mathrm{~d}, J=2.0 \mathrm{~Hz}, 1 \mathrm{H}), 7.48(\mathrm{t}, J=$ $8.0 \mathrm{~Hz}, 1 \mathrm{H}), 7.64(\mathrm{t}, J=7.6 \mathrm{~Hz}, 1 \mathrm{H}), 8.06(\mathrm{t}, J=6.0 \mathrm{~Hz}, 1 \mathrm{H}) ; 8.15(\mathrm{~s}, 1 \mathrm{H}) ;{ }^{13} \mathrm{CNMR}\left(100 \mathrm{MHz}, \mathrm{CDCl}_{3}\right) \delta(\mathrm{ppm})$ 24.6, 25.8, 27.7, 42.4, 56.6, 69.7, 120.8, 121.9, 129.0, 131.9, 143.9, 148.1, 214.0; HPLC analysis Chiralcel AD-H (Hexane/ i-PrOH $=95 / 5,1.0 \mathrm{~mL} / \mathrm{min}, 254 \mathrm{~nm}, 20{ }^{\circ} \mathrm{C}$ ) $\mathrm{t}_{R} 30.4 \mathrm{~min}$ and $\mathrm{t}_{R} 33.3 \mathrm{~min}$.

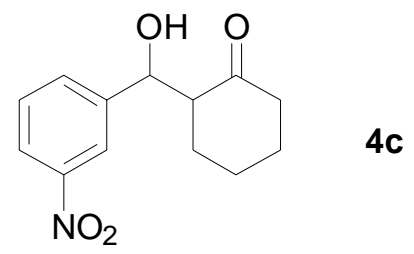

2-[Hydroxy-(4-cyano-phenyl)-methyl]-cyclohexanone (4d): Reaction time: $21 \mathrm{~h}$; yield: 89\%; Anti/Syn= 97/3, anti-diastereomer, ${ }^{1} \mathrm{HNMR}\left(400 \mathrm{MHz}, \mathrm{CDCl}_{3}\right) \delta(\mathrm{ppm}) 1.31-2.11(\mathrm{~m}, 6 \mathrm{H}), 2.30-2.48(\mathrm{~m}, 2 \mathrm{H}), 2.53-2.59(\mathrm{~m}, 1 \mathrm{H})$, $4.07(\mathrm{~s}, 1 \mathrm{H}), 4.82(\mathrm{~d}, J=8.4 \mathrm{~Hz}, 1 \mathrm{H}), 7.43(\mathrm{~d}, J=8.4 \mathrm{~Hz}, 2 \mathrm{H}), 7.62(\mathrm{~d}, J=8.0 \mathrm{~Hz}, 2 \mathrm{H}) ;{ }^{13} \mathrm{CNMR}(100 \mathrm{MHz}$, $\left.\mathrm{CDCl}_{3}\right) \delta$ (ppm) 24.6, 27.6, 30.6, 42.5, 57.0, 74.0, 111.5, 118.6, 127.7, 132.1, 146.3, 214.7; HPLC analysis Chiralcel OD-H (Hexane/ i-PrOH $=95 / 5,1.0 \mathrm{~mL} / \mathrm{min}, 220 \mathrm{~nm}, 20{ }^{\circ} \mathrm{C}$ ) $\mathrm{t}_{R}$ (minor) $50.2 \mathrm{~min}$ and $\mathrm{t}_{R}$ (major) $64.0 \mathrm{~min}$, ee: 92\%; syn-diastereomer, ${ }^{1} \mathrm{HNMR}\left(400 \mathrm{MHz}, \mathrm{CDCl}_{3}\right) \delta(\mathrm{ppm})$ 1.52-2.12 (m, 6H), 2.33-2.48 (m, 2H), 2.57-2.61 (m, 1H), 3.19 (s, 1H), $5.42(\mathrm{~s}, 1 \mathrm{H}), 7.42(\mathrm{~d}, J=8.0 \mathrm{~Hz}, 2 \mathrm{H}), 7.62(\mathrm{~d}, J=8.4 \mathrm{~Hz}, 2 \mathrm{H}) ;{ }^{13} \mathrm{CNMR}\left(100 \mathrm{MHz}, \mathrm{CDCl}_{3}\right)$ $\delta$ (ppm) 24.7, 25.8, 27.8, 42.5, 56.7, 70.1, 110.7, 118.8, 126.5, 132.0, 147.0, 214.1; HPLC analysis Chiralcel OD-H (Hexane/ i-PrOH $=95 / 5,1.0 \mathrm{~mL} / \mathrm{min}, 220 \mathrm{~nm}, 20^{\circ} \mathrm{C}$ ) $\mathrm{t}_{R} 34.2 \mathrm{~min}$ and $\mathrm{t}_{R} 42.6 \mathrm{~min}$.

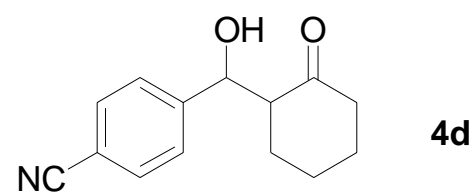

2-[Hydroxy-(4-chloro-phenyl)-methyl]-cyclohexanone (4e): Reaction time: $48 \mathrm{~h}$; yield: 88\%; Anti/Syn= 96/4, anti-diastereomer, ${ }^{1} \mathrm{HNMR}\left(400 \mathrm{MHz}, \mathrm{CDCl}_{3}\right) \delta(\mathrm{ppm})$ 1.26-2.11 (m, 6H), 2.31-2.49 (m, 2H), 2.52-2.59 (m, 1H), $3.61(\mathrm{~s}, 1 \mathrm{H}), 4.76(\mathrm{~d}, J=8.4 \mathrm{~Hz}, 1 \mathrm{H}), 7.25(\mathrm{~d}, J=8.4 \mathrm{~Hz}, 2 \mathrm{H}), 7.31(\mathrm{~d}, J=8.8 \mathrm{~Hz}, 2 \mathrm{H}) ;{ }^{13} \mathrm{CNMR}(100 \mathrm{MHz}$, $\left.\mathrm{CDCl}_{3}\right) \delta(\mathrm{ppm}) 24.6,27.2,30.7,42.6,57.3,74.0,128.3,128.4,133.5,139.5,215.2$; HPLC analysis Chiralcel OD-H (Hexane/ i-PrOH = 95/5, $1.0 \mathrm{~mL} / \mathrm{min}, 220 \mathrm{~nm}, 20{ }^{\circ} \mathrm{C}$ ) $\mathrm{t}_{R}$ (minor) $23.2 \mathrm{~min}$ and $\mathrm{t}_{R}$ (major) $27.4 \mathrm{~min}$, ee: 94\%; syn-diastereomer, ${ }^{1} \mathrm{HNMR}\left(400 \mathrm{MHz}, \mathrm{CDCl}_{3}\right) \delta(\mathrm{ppm})$ 1.49-2.09 (m, 6H), 2.32-2.46 (m, 2H), 2.53-2.57 (m, 1H), $2.92(\mathrm{~s}, 1 \mathrm{H}), 5.34(\mathrm{~d}, J=2.0 \mathrm{~Hz}, 1 \mathrm{H}), 7.23(\mathrm{~d}, J=8.4 \mathrm{~Hz}, 2 \mathrm{H}), 7.30(\mathrm{~d}, J=8.4 \mathrm{~Hz}, 2 \mathrm{H}) ;{ }^{13} \mathrm{CNMR}(100 \mathrm{MHz}$, $\left.\mathrm{CDCl}_{3}\right) \delta(\mathrm{ppm}) 24.8,25.9,27.8,42.6,57.0,70.0,127.1,128.2,132.6,140.0,214.5$; HPLC analysis Chiralcel OD-H (Hexane/ i-PrOH $\left.=95 / 5,1.0 \mathrm{~mL} / \mathrm{min}, 220 \mathrm{~nm}, 20^{\circ} \mathrm{C}\right) \mathrm{t}_{R} 13.5 \mathrm{~min}$ and $\mathrm{t}_{R} 16.6 \mathrm{~min}$.

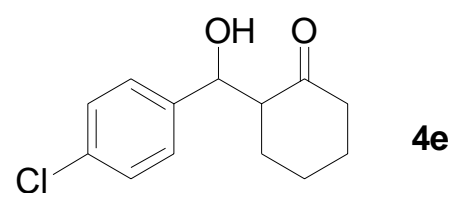

2-[Hydroxy-(2-chloro-phenyl)-methyl]-cyclohexanone (4f): Reaction time: $50 \mathrm{~h}$; yield: 83\%; Anti/Syn= 97/3, anti-diastereomer, ${ }^{1} \mathrm{HNMR}\left(400 \mathrm{MHz}, \mathrm{CDCl}_{3}\right) \delta(\mathrm{ppm})$ 1.51-2.09 (m, 6H), 2.28-2.46 (m, 2H), 2.63-2.70 (m, 1H), $3.92(\mathrm{~s}, 1 \mathrm{H}), 5.34(\mathrm{~d}, J=8.0 \mathrm{~Hz}, 1 \mathrm{H}), 7.19(\mathrm{t}, J=7.6 \mathrm{~Hz}, 1 \mathrm{H}), 7.28(\mathrm{~d}, J=7.6 \mathrm{~Hz}, 1 \mathrm{H}), 7.31(\mathrm{~d}, J=8.0 \mathrm{~Hz}, 1 \mathrm{H})$, 
$7.53(\mathrm{~d}, J=7.6 \mathrm{~Hz}, 1 \mathrm{H}) ;{ }^{13} \mathrm{CNMR}\left(100 \mathrm{MHz}, \mathrm{CDCl}_{3}\right) \delta(\mathrm{ppm}) 24.8,27.7,30.3,42.6,57.5,70.3,127.1,128.2$, 128.6, 129.1 132.8, 139.0, 215.1; HPLC analysis Chiralcel OD-H (Hexane/ i-PrOH = 95/5, $1.0 \mathrm{~mL} / \mathrm{min}, 220 \mathrm{~nm}$, $\left.20{ }^{\circ} \mathrm{C}\right) \mathrm{t}_{R}$ (major) $17.2 \mathrm{~min}$ and $\mathrm{t}_{R}$ (minor) $19.7 \mathrm{~min}$, ee: 92\%; syn-diastereomer, ${ }^{1} \mathrm{HNMR}\left(400 \mathrm{MHz}, \mathrm{CDCl}_{3}\right) \delta$ (ppm) $1.49-2.10(\mathrm{~m}, 6 \mathrm{H}), 2.35-2.48(\mathrm{~m}, 2 \mathrm{H}), 2.78-2.83(\mathrm{~m}, 1 \mathrm{H}), 3.16(\mathrm{~s}, 1 \mathrm{H}), 5.71(\mathrm{~d}, J=1.2 \mathrm{~Hz}, 1 \mathrm{H}), 7.19(\mathrm{t}, J=$ $8.0 \mathrm{~Hz}, 1 \mathrm{H}), 7.27(\mathrm{~d}, J=4.4 \mathrm{~Hz}, 1 \mathrm{H}), 7.31(\mathrm{~d}, J=8.0 \mathrm{~Hz}, 1 \mathrm{H}), 7.56(\mathrm{~d}, J=8.0 \mathrm{~Hz}, 1 \mathrm{H}) ;{ }^{13} \mathrm{CNMR}(100 \mathrm{MHz}$, $\left.\mathrm{CDCl}_{3}\right) \delta(\mathrm{ppm}) 24.7,25.8,27.8,42.5,53.5,67.5,126.5,128.1,128.4,129.1,130.7,138.6,214.5$; HPLC analysis Chiralcel OD-H (Hexane/ i-PrOH $\left.=95 / 5,1.0 \mathrm{~mL} / \mathrm{min}, 220 \mathrm{~nm}, 20{ }^{\circ} \mathrm{C}\right) \mathrm{t}_{R} 9.0 \mathrm{~min}$ and $\mathrm{t}_{R} 10.2 \mathrm{~min}$.

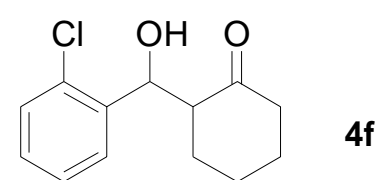

2-[Hydroxy-(4-bromo-phenyl)-methyl]-cyclohexanone (4g): Reaction time: $52 \mathrm{~h}$; yield: 76\%; Anti/Syn= 98/2, anti-diastereomer, ${ }^{1} \mathrm{HNMR}\left(400 \mathrm{MHz}, \mathrm{CDCl}_{3}\right) \delta(\mathrm{ppm})$ 1.24-2.08 (m, 6H), 2.28-2.37 (m, 2H), 2.43-2.57 (m, 1H), $3.40(\mathrm{~s}, 1 \mathrm{H}), 4.73$ (d, $J=8.4 \mathrm{~Hz}, 1 \mathrm{H}), 7.18(\mathrm{~d}, J=8.0 \mathrm{~Hz}, 2 \mathrm{H}), 7.45$ (d, $J=8.4 \mathrm{~Hz}, 2 \mathrm{H}) ;{ }^{13} \mathrm{CNMR}(100 \mathrm{MHz}$, $\left.\mathrm{CDCl}_{3}\right) \delta(\mathrm{ppm}) 24.6,27.6,30.6,42.5,57.2,74.0,121.6,128.6,131.3,140.0,215.1$; HPLC analysis Chiralcel OD-H (Hexane/ i-PrOH = 95/5, $1.0 \mathrm{~mL} / \mathrm{min}, 220 \mathrm{~nm}, 20{ }^{\circ} \mathrm{C}$ ) $\mathrm{t}_{R}$ (minor) $24.2 \mathrm{~min}$ and $\mathrm{t}_{R}$ (major) $29.0 \mathrm{~min}$, ee: 97\%; syn-diastereomer, ${ }^{1} \mathrm{HNMR}\left(400 \mathrm{MHz}, \mathrm{CDCl}_{3}\right) \delta(\mathrm{ppm})$ 1.46-2.08 (m, 6H), 2.30-2.45 (m, 2H), 2.50-2.56 (m, $\left.1 \mathrm{H}\right)$, $3.04(\mathrm{~s}, 1 \mathrm{H}), 5.31(\mathrm{~d}, J=2.4 \mathrm{~Hz}, 1 \mathrm{H}), 7.12(\mathrm{~d}, J=8.4 \mathrm{~Hz}, 2 \mathrm{H}), 7.44(\mathrm{~d}, J=8.4 \mathrm{~Hz}, 2 \mathrm{H}) ;{ }^{13} \mathrm{CNMR}(100 \mathrm{MHz}$, $\left.\mathrm{CDCl}_{3}\right) \delta(\mathrm{ppm}) 24.7,25.8,27.8,42.5,56.9,70.0,120.6,127.5,131.1,140.5,214.4$; HPLC analysis Chiralcel OD-H (Hexane/ i-PrOH $=95 / 5,1.0 \mathrm{~mL} / \mathrm{min}, 220 \mathrm{~nm}, 20^{\circ} \mathrm{C}$ ) $\mathrm{t}_{R} 13.7 \mathrm{~min}$ and $\mathrm{t}_{R} 17.1 \mathrm{~min}$.

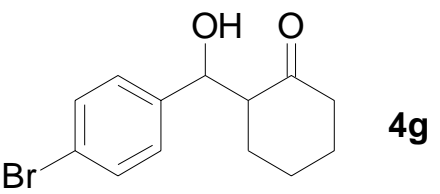

2-(Hydroxy-phenyl-methyl)-cyclohexanone (4h): Reaction time: $72 \mathrm{~h}$; yield: 73\%; Anti/Syn= 83/17, anti-diastereomer, ${ }^{1} \mathrm{HNMR}\left(400 \mathrm{MHz}, \mathrm{CDCl}_{3}\right) \delta(\mathrm{ppm}) 1.25-2.10(\mathrm{~m}, 6 \mathrm{H}), 2.32-2.49(\mathrm{~m}, 2 \mathrm{H}), 2.58-2.65(\mathrm{~m}, 1 \mathrm{H})$, $4.78(\mathrm{~d}, J=6.0 \mathrm{~Hz}, 1 \mathrm{H}), 7.29-7.36(\mathrm{~m}, 5 \mathrm{H}) ;{ }^{13} \mathrm{CNMR}\left(100 \mathrm{MHz}, \mathrm{CDCl}_{3}\right) \delta(\mathrm{ppm}) 24.7,27.8,30.8,42.6,57.4,74.7$, 127.0, 127.9, 128.3, 140.9, 215.5; HPLC analysis Chiralcel OD-H (Hexane/ i-PrOH = 90/10, $1.0 \mathrm{~mL} / \mathrm{min}, 220 \mathrm{~nm}$, $\left.20{ }^{\circ} \mathrm{C}\right) \mathrm{t}_{R}$ (major) $18.5 \mathrm{~min}$ and $\mathrm{t}_{R}$ (major) $27.5 \mathrm{~min}$, ee: 87\%; syn-diastereomer, ${ }^{1} \mathrm{HNMR}\left(400 \mathrm{MHz}, \mathrm{CDCl}_{3}\right) \delta$ (ppm) 1.48-2.10 (m, 6H), 2.32-2.47 (m, 2H), 2.57-2.62 (m, 1H), 5.39 (d, J = 2.4 Hz, 1H), 7.23-7.35 (m, 5H); ${ }^{13} \mathrm{CNMR}\left(100 \mathrm{MHz}, \mathrm{CDCl}_{3}\right) \delta$ (ppm) 24.8, 26.0, 27.9, 42.6, 57.1, 70.6, 125.7, 126.9, 128.1, 141.4, 214.8; HPLC analysis Chiralcel OD-H (Hexane/ $\mathrm{i}-\mathrm{PrOH}=90 / 10,1.0 \mathrm{~mL} / \mathrm{min}, 220 \mathrm{~nm}, 20^{\circ} \mathrm{C}$ ) $\mathrm{t}_{R} 14.8 \mathrm{~min}$ and $\mathrm{t}_{R} 16.3 \mathrm{~min}$.<smiles>O=C1CCCCC1C(O)c1ccccc1</smiles>

2-(Hydroxy-naphthyl-methyl)-cyclohexanone (4i): Reaction time: $72 \mathrm{~h}$; yield: 41\%; Anti/Syn= 97/3, anti-diastereomer, ${ }^{1} \mathrm{HNMR}\left(400 \mathrm{MHz}, \mathrm{CDCl}_{3}\right) \delta(\mathrm{ppm}) 1.27-2.10(\mathrm{~m}, 6 \mathrm{H}), 2.39-2.53(\mathrm{~m}, 2 \mathrm{H}), 2.98-3.01(\mathrm{~m}, 1 \mathrm{H})$, $4.17(\mathrm{~s}, 1 \mathrm{H}), 5.59$ (d, $J=8.8 \mathrm{~Hz}, 1 \mathrm{H}), 7.45-7.51(\mathrm{~m}, 3 \mathrm{H}), 7.57(\mathrm{br} \mathrm{d}, J=7.2 \mathrm{~Hz}, 1 \mathrm{H}), 7.81(\mathrm{~d}, \mathrm{~J}=8.0 \mathrm{~Hz}, 1 \mathrm{H})$, 7.86-7.89 (m, 1H), $8.26(\mathrm{~d}, \mathrm{~J}=8.3 \mathrm{~Hz}, 1 \mathrm{H}) ;{ }^{13} \mathrm{CNMR}\left(100 \mathrm{MHz}, \mathrm{CDCl}_{3}\right) \delta(\mathrm{ppm}) 24.8,27.9,31.3,42.7,57.3,72.0$, $123.9,125.2,125.3,125.4,125.9,128.4,128.8,131.3,133.8,136.7,215.7$; HPLC analysis Chiralcel OJ-H (Hexane/ i-PrOH $=90 / 10,0.5 \mathrm{~mL} / \mathrm{min}, 220 \mathrm{~nm}, 20{ }^{\circ} \mathrm{C}$ ) $\mathrm{t}_{R}$ (minor) $21.8 \mathrm{~min}$ and $\mathrm{t}_{R}$ (major) $24.1 \mathrm{~min}$, ee: 90\%; syn-diastereomer, HPLC analysis Chiralcel OJ-H (Hexane/ $\left.\mathrm{i}-\mathrm{PrOH}=90 / 10,0.5 \mathrm{~mL} / \mathrm{min}, 220 \mathrm{~nm}, 20{ }^{\circ} \mathrm{C}\right) \mathrm{t}_{R}$ $17.4 \mathrm{~min}$ and $\mathrm{t}_{R} 18.3 \mathrm{~min}$. 


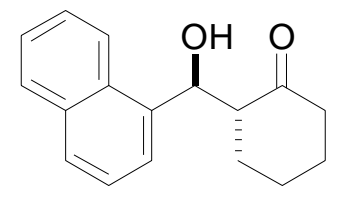

2-(Furan-2-yl-hydroxy-methyl)-cyclohexanone (4j): Reaction time: $72 \mathrm{~h}$; yield: 53\%; Anti/Syn= 87/13, anti-diastereomer, ${ }^{1} \mathrm{HNMR}\left(400 \mathrm{MHz}, \mathrm{CDCl}_{3}\right) \delta(\mathrm{ppm}) 1.30-2.10(\mathrm{~m}, 6 \mathrm{H}), 2.34-2.46(\mathrm{~m}, 2 \mathrm{H}), 2.88-2.90(\mathrm{~m}, 1 \mathrm{H})$, $3.90(\mathrm{~d}, J=3.6 \mathrm{~Hz}, 1 \mathrm{H}), 4.81(\mathrm{~d}, J=8.4 \mathrm{~Hz}, 1 \mathrm{H}), 6.26(\mathrm{~d}, J=3.2 \mathrm{~Hz}, 1 \mathrm{H}), 6.30(\mathrm{q}, J=2.8 \mathrm{~Hz}, 1 \mathrm{H}), 7.36(\mathrm{~d}, J=$ $1.2 \mathrm{~Hz}, 1 \mathrm{H}) ;{ }^{13} \mathrm{CNMR}\left(100 \mathrm{MHz}, \mathrm{CDCl}_{3}\right) \delta$ (ppm) 24.6, 27.7, 30.5, 42.5, 54.8, 68.1, 107.9, 110.0, 142.2, 153.5, 214.9; HPLC analysis Chiralcel AD-H (Hexane/ i-PrOH =95/5, $1.0 \mathrm{~mL} / \mathrm{min}, 220 \mathrm{~nm}, 20{ }^{\circ} \mathrm{C}$ ) $\mathrm{t}_{R}$ (major) $24.5 \mathrm{~min}$ and $\mathrm{t}_{R}$ (minor) $27.1 \mathrm{~min}$, ee: 93\%; syn-diastereomer, ${ }^{1} \mathrm{HNMR}\left(400 \mathrm{MHz}, \mathrm{CDCl}_{3}\right) \delta(\mathrm{ppm}){ }^{13} \mathrm{CNMR}(100 \mathrm{MHz}$, $\left.\mathrm{CDCl}_{3}\right) \delta(\mathrm{ppm}) 1.61-2.10(\mathrm{~m}, 6 \mathrm{H}), 2.34-2.45(\mathrm{~m}, 2 \mathrm{H}), 2.78-2.82(\mathrm{~m}, 1 \mathrm{H}), 3.11(\mathrm{~d}, J=4.8 \mathrm{~Hz}, 1 \mathrm{H}), 5.26(\mathrm{t}, J=3.6$ $\mathrm{Hz}, 1 \mathrm{H}), 6.26(\mathrm{~d}, J=2.8 \mathrm{~Hz}, 1 \mathrm{H}), 6.30(\mathrm{q}, J=2.8 \mathrm{~Hz}, 1 \mathrm{H}), 7.33(\mathrm{~d}, J=1.6 \mathrm{~Hz}, 1 \mathrm{H}) ;{ }^{13} \mathrm{CNMR}\left(100 \mathrm{MHz}, \mathrm{CDCl}_{3}\right)$ $\delta(\mathrm{ppm}) 24.7,27.2,27.5,42.4,54.5,66.5,106.5,110.2$, 141.4, 154.6, 213.6; HPLC analysis Chiralcel AD-H (Hexane/ $\mathrm{i}-\mathrm{PrOH}=95 / 5,1.0 \mathrm{~mL} / \mathrm{min}, 220 \mathrm{~nm}, 20^{\circ} \mathrm{C}$ ) $\mathrm{t}_{R} 17.0 \mathrm{~min}$ and $\mathrm{t}_{R} 18.7 \mathrm{~min}$.

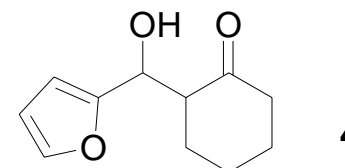

2-[Hydroxy-(4-methyl-phenyl)-methyl]-cyclohexanone (4k): Reaction time: 100 h; yield: 53\%; Anti/Syn= 80/20, anti-diastereomer, ${ }^{1} \mathrm{HNMR}\left(400 \mathrm{MHz}, \mathrm{CDCl}_{3}\right) \delta(\mathrm{ppm})$ 1.52-2.10 (m, 6H), $2.34(\mathrm{~s}, 3 \mathrm{H}), 2.36-2.49(\mathrm{~m}, 2 \mathrm{H})$, 2.57-2.64 (m, 1H), $3.94(\mathrm{~s}, 1 \mathrm{H}), 4.75(\mathrm{~d}, J=8.8 \mathrm{~Hz}, 1 \mathrm{H}), 7.15(\mathrm{~d}, J=8.0 \mathrm{~Hz}, 2 \mathrm{H}), 7.21(\mathrm{~d}, J=7.6 \mathrm{~Hz}, 2 \mathrm{H})$; ${ }^{13} \mathrm{CNMR}\left(100 \mathrm{MHz}, \mathrm{CDCl}_{3}\right) \delta$ (ppm) 21.1, 24.6, 27.8, 29.6, 42.6, 57.4, 74.4, 126.9, 129.0, 137.4, 137.9, 215.5; HPLC analysis Chiralcel OD-H (Hexane/ i-PrOH =97/3, $1.0 \mathrm{~mL} / \mathrm{min}, 220 \mathrm{~nm}, 20{ }^{\circ} \mathrm{C}$ ) $\mathrm{t}_{R}$ (major) 29.5 min and $\mathrm{t}_{R}$ (minor) $41.4 \mathrm{~min}$, ee: 77\%; syn-diastereomer, ${ }^{1} \mathrm{HNMR}$ (400 MHz, $\left.\mathrm{CDCl}_{3}\right) \delta(\mathrm{ppm})$ 1.50-2.10 (m, 6H), $2.34(\mathrm{~s}, 3 \mathrm{H})$, 2.36-2.46 (m, 2H), 2.55-2.60 (m, 1H), $2.97(\mathrm{~s}, 1 \mathrm{H}), 5.35$ (d, $J=1.6 \mathrm{~Hz}, 1 \mathrm{H}), 7.15(\mathrm{~d}, J=8.0 \mathrm{~Hz}, 2 \mathrm{H}), 7.19$ (d, $J=$ $8.0 \mathrm{~Hz}, 2 \mathrm{H}) ;{ }^{13} \mathrm{CNMR}\left(100 \mathrm{MHz}, \mathrm{CDCl}_{3}\right) \delta$ (ppm) 21.1, 24.9, 26.1, 28.0, 42.7, 57.2, 70.5, 125.7, 128.8, 136.5, 138.4, 214.9; HPLC analysis Chiralcel OD-H (Hexane/ i-PrOH =97/3, $1.0 \mathrm{~mL} / \mathrm{min}, 220 \mathrm{~nm}, 20{ }^{\circ} \mathrm{C}$ ) $\mathrm{t}_{R} 21.1 \mathrm{~min}$ and $\mathrm{t}_{R} 22.6 \mathrm{~min}$.

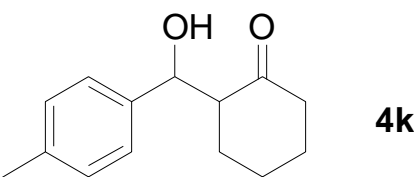

4-Hydroxy-3-methyl-4-(4-nitro-phenyl)-butan-2-one (6): Reaction time: 72 h; Yield: 63\%; Anti/Syn= 95/5, anti-diastereomer, ${ }^{1} \mathrm{HNMR}\left(400 \mathrm{MHz}, \mathrm{CDCl}_{3}\right) \delta(\mathrm{ppm}) 1.01(\mathrm{~d}, J=7.6 \mathrm{~Hz} 3 \mathrm{H}), 2.21(\mathrm{~s}, 3 \mathrm{H}), 2.87-2.94(\mathrm{~m}, 1 \mathrm{H})$, $3.34(\mathrm{~d}, J=4.8 \mathrm{~Hz}, 1 \mathrm{H}), 4.87(\mathrm{~d}, J=8.0 \mathrm{~Hz}, 1 \mathrm{H}), 7.51(\mathrm{~d}, J=8.4 \mathrm{~Hz}, 2 \mathrm{H}), 8.21(\mathrm{~d}, J=8.8 \mathrm{~Hz}, 2 \mathrm{H}) ;{ }^{13} \mathrm{CNMR}(100$ $\left.\mathrm{MHz}, \mathrm{CDCl}_{3}\right) \delta(\mathrm{ppm})$ 14.1, 29.7, 53.2, 75.4, 123.7, 127.5, 147.5, 149.3, 212.8; HPLC analysis Chiralcel AS-H (Hexane/ i-PrOH = 93/7, $1.0 \mathrm{~mL} / \mathrm{min}, 254 \mathrm{~nm}, 20{ }^{\circ} \mathrm{C}$ ) $\mathrm{t}_{R}$ (minor) $47.4 \mathrm{~min}$ and $\mathrm{t}_{R}$ (major) $52.9 \mathrm{~min}$. syn-diastereomer, HPLC analysis Chiralcel AS-H (Hexane/ i-PrOH =93/7, $1.0 \mathrm{~mL} / \mathrm{min}, 254 \mathrm{~nm}, 20{ }^{\circ} \mathrm{C}$ ) $\mathrm{t}_{R} 64.5$ $\min$ and $\mathrm{t}_{R} 72.7 \mathrm{~min}$.

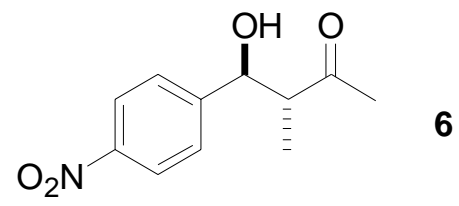


NMR Spectra
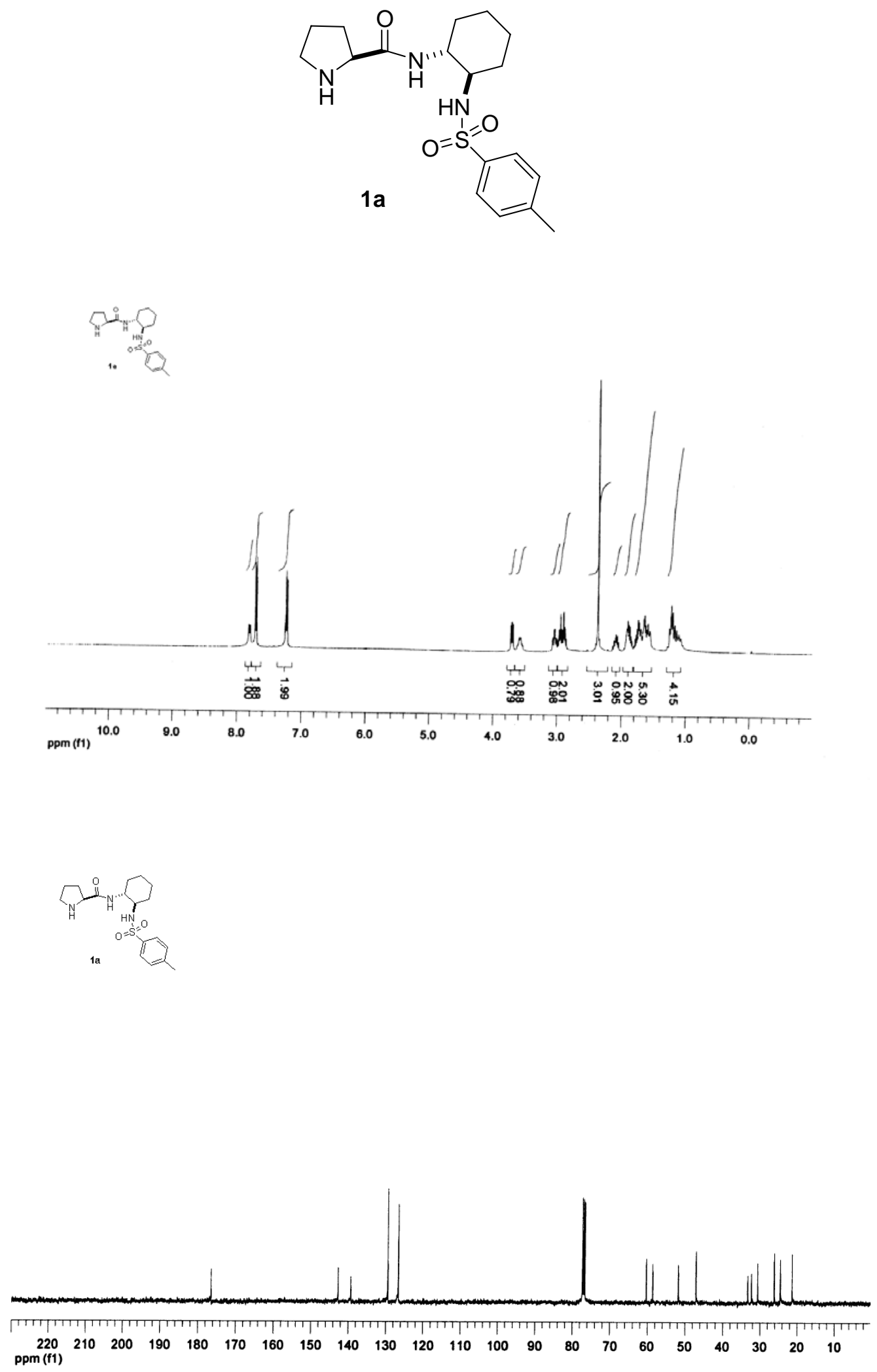

Sq 

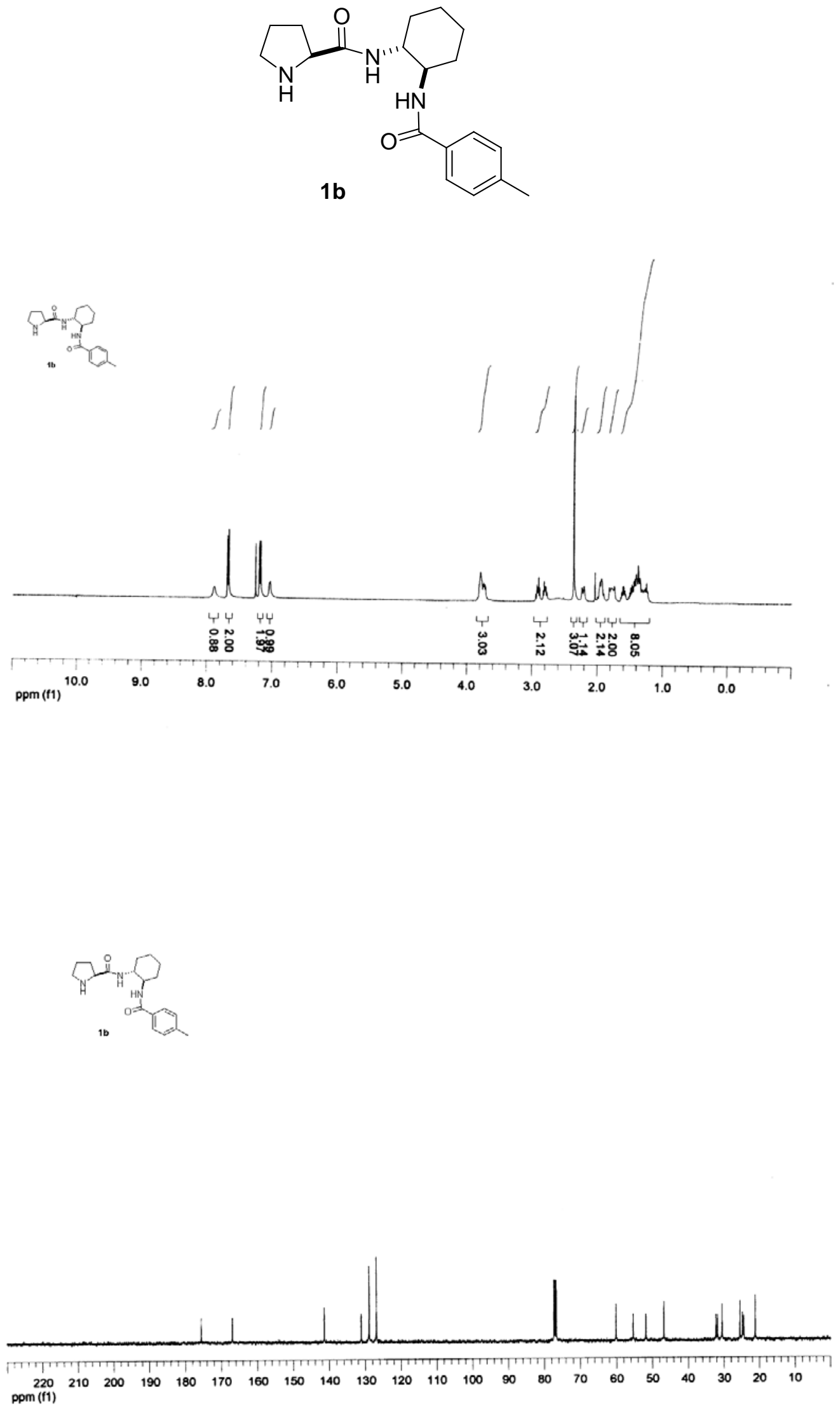

S10 

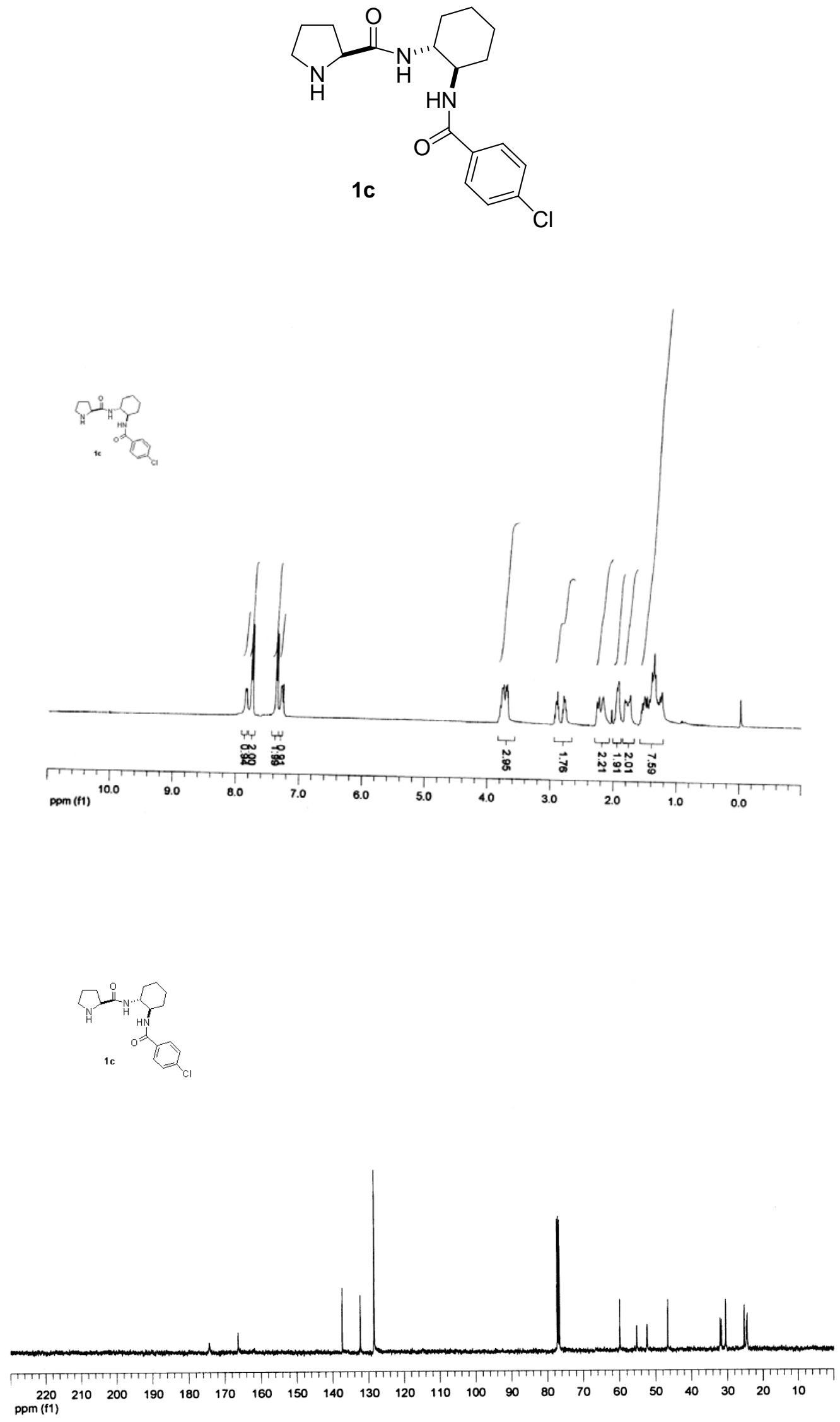

S11 

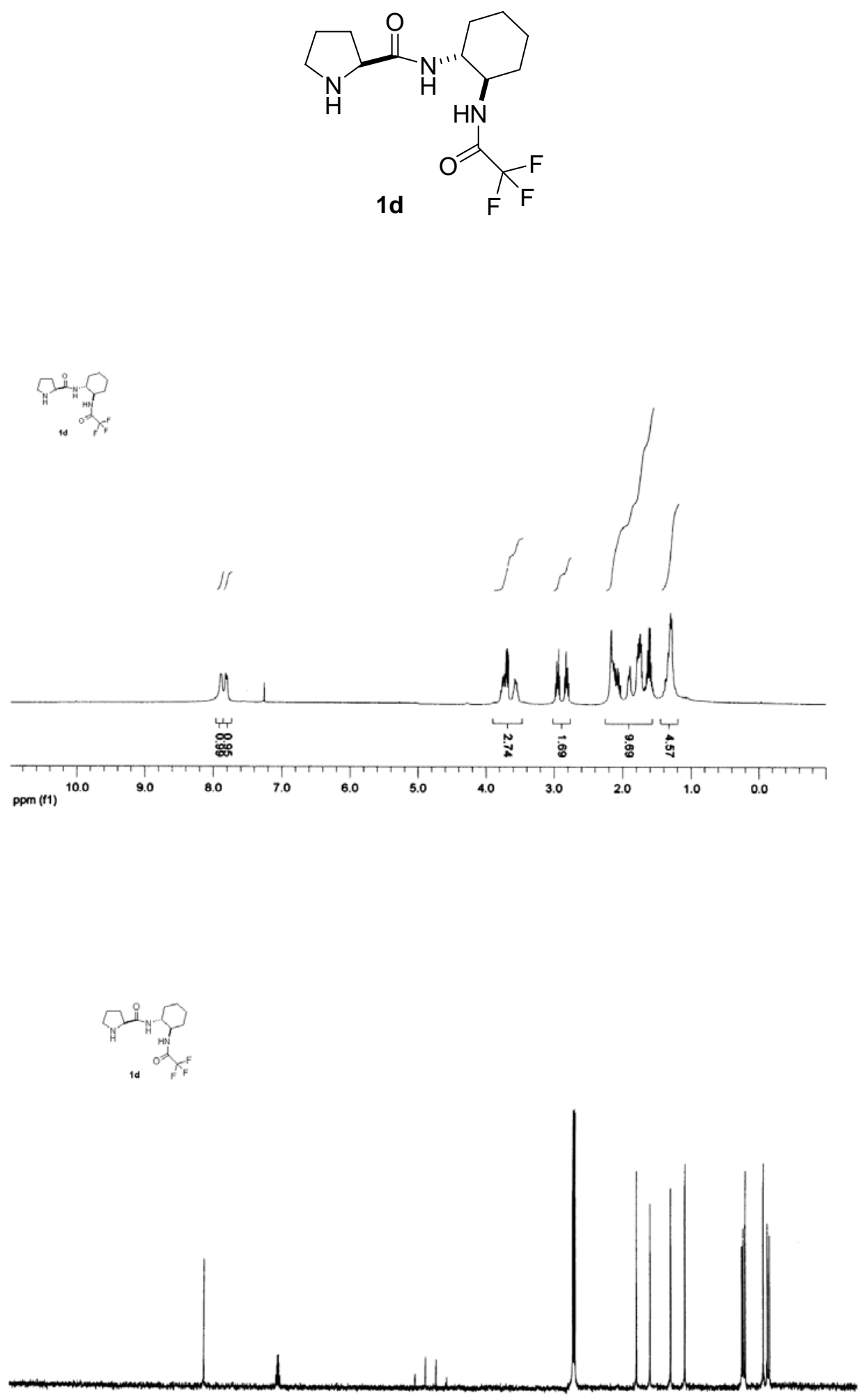

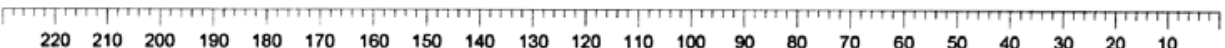
$\begin{array}{clllllllllllllllllllll}220 & 210 & 200 & 190 & 180 & 170 & 160 & 150 & 140 & 130 & 120 & 110 & 100 & 90 & 80 & 70 & 60 & 50 & 40 & 30 & 20 & 10 \\ \operatorname{ppm}(\mathrm{f} 1) & & & & & & & & & \end{array}$ 


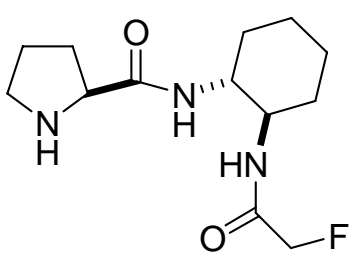

$1 e$
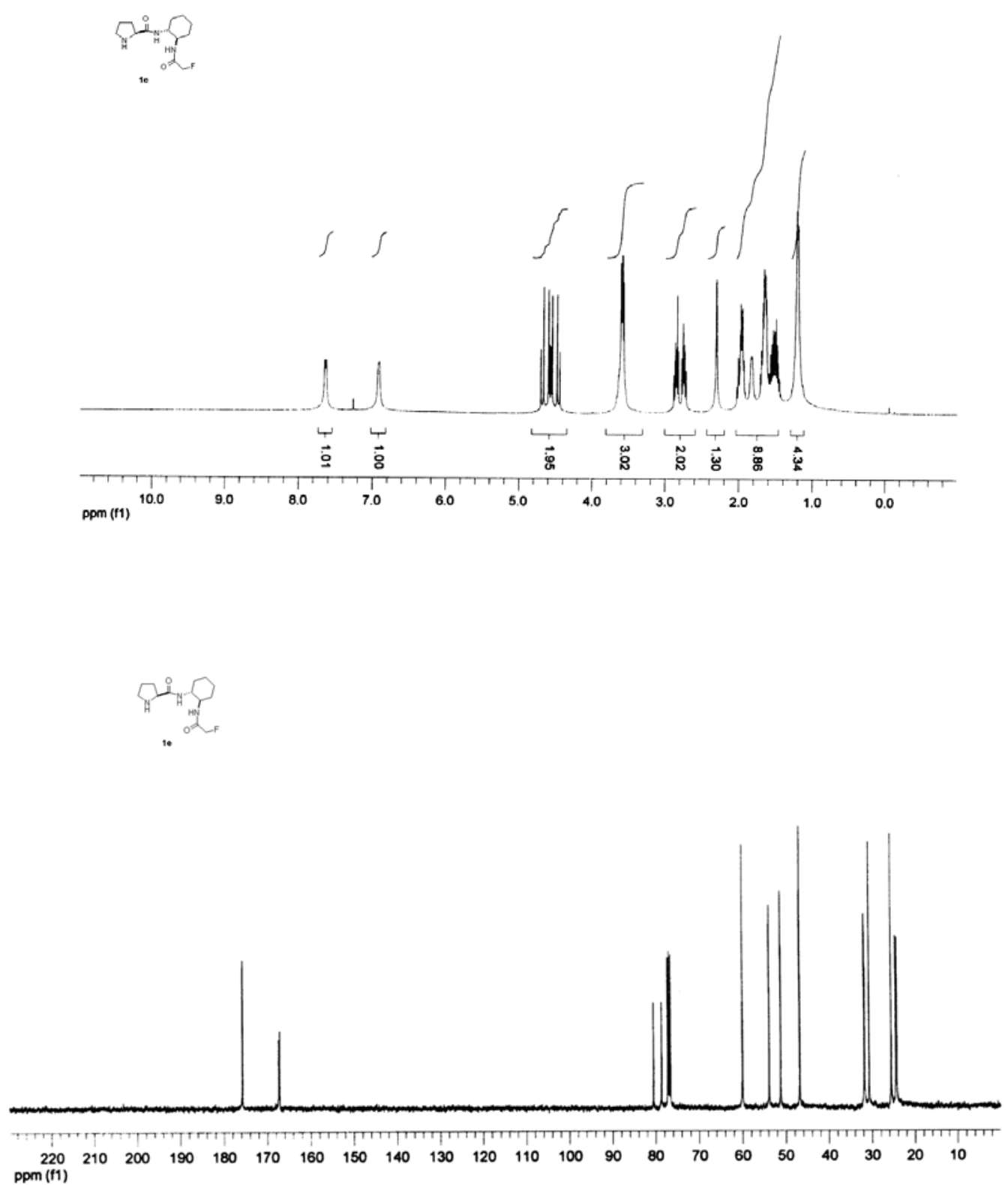

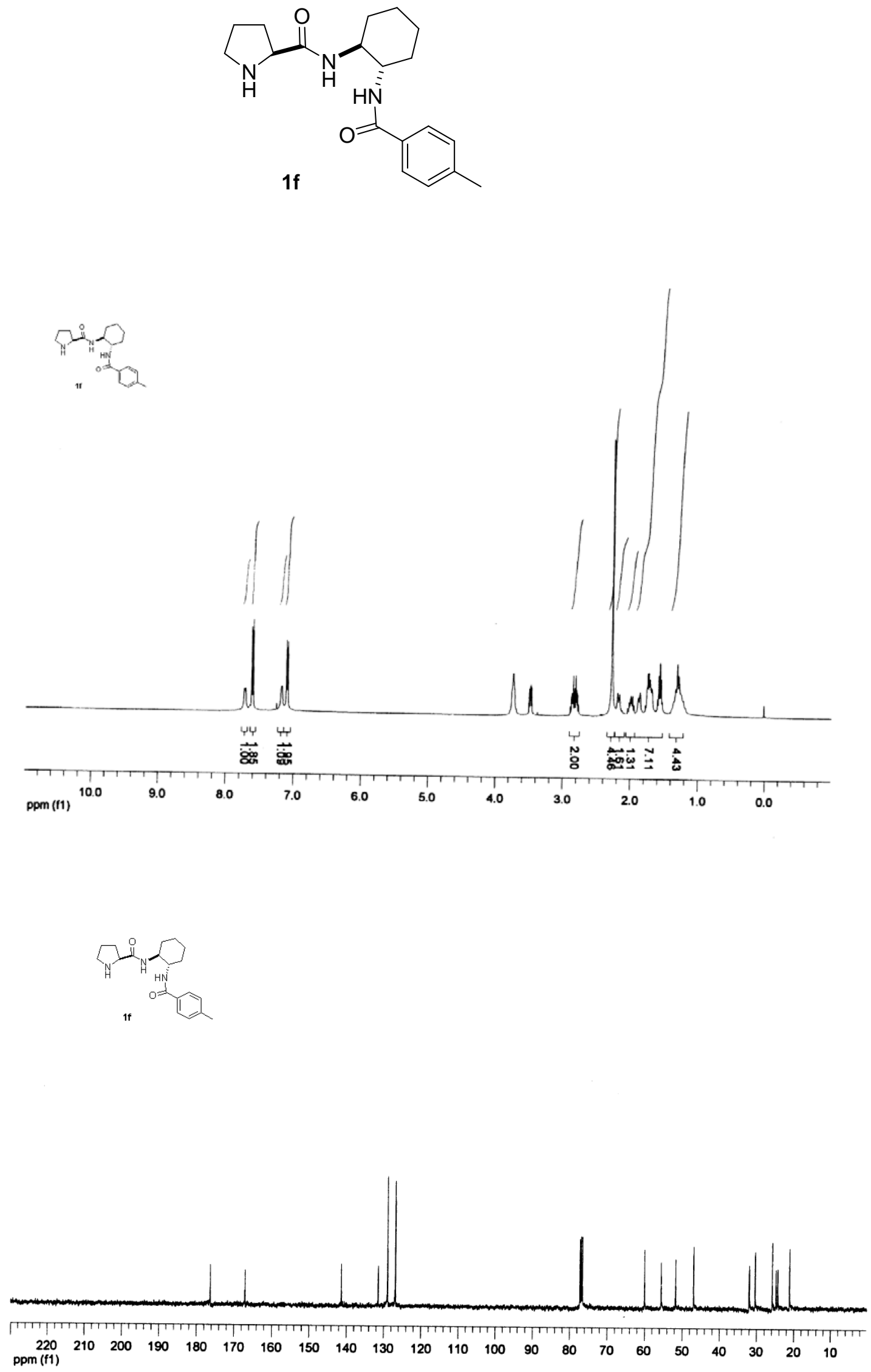

S14 
<smiles>O=C1CCCCC1C(O)c1ccc([N+](=O)[O-])cc1</smiles>

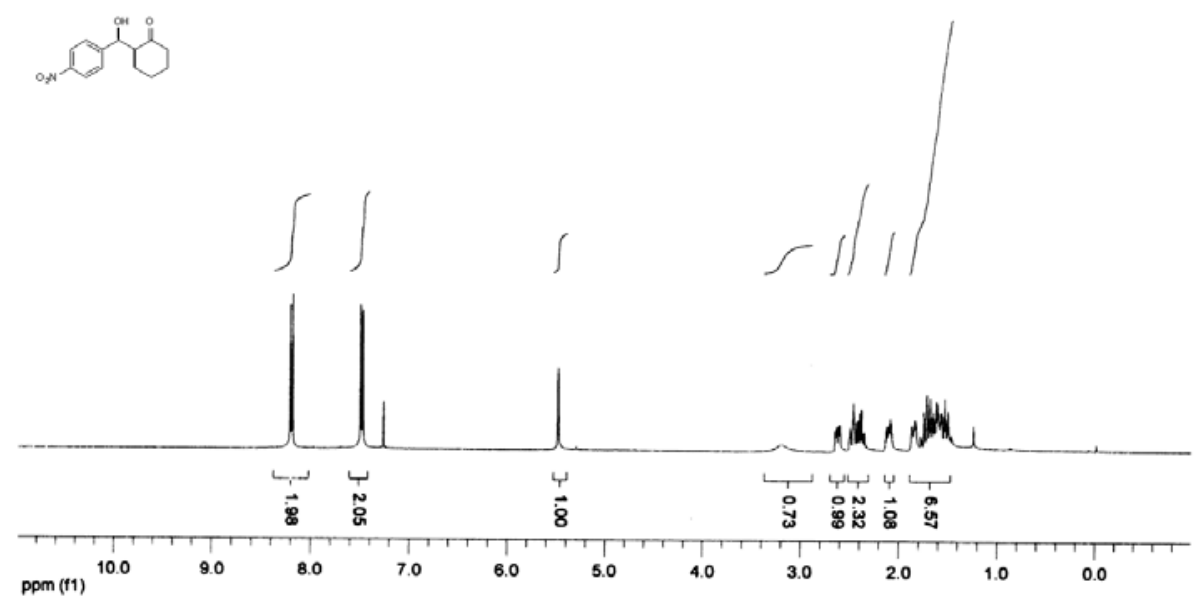

or

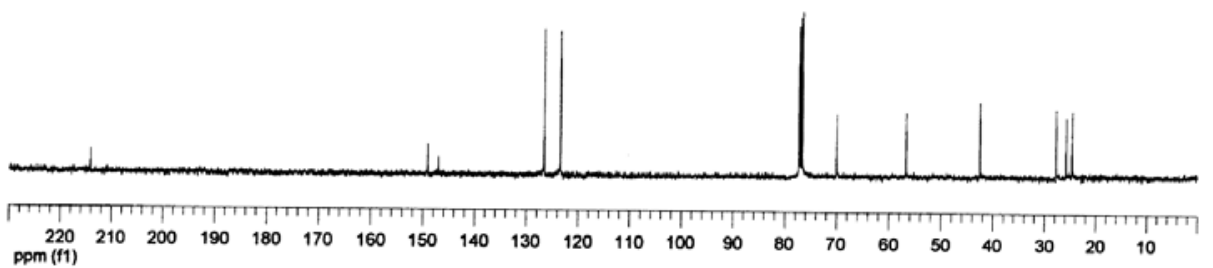



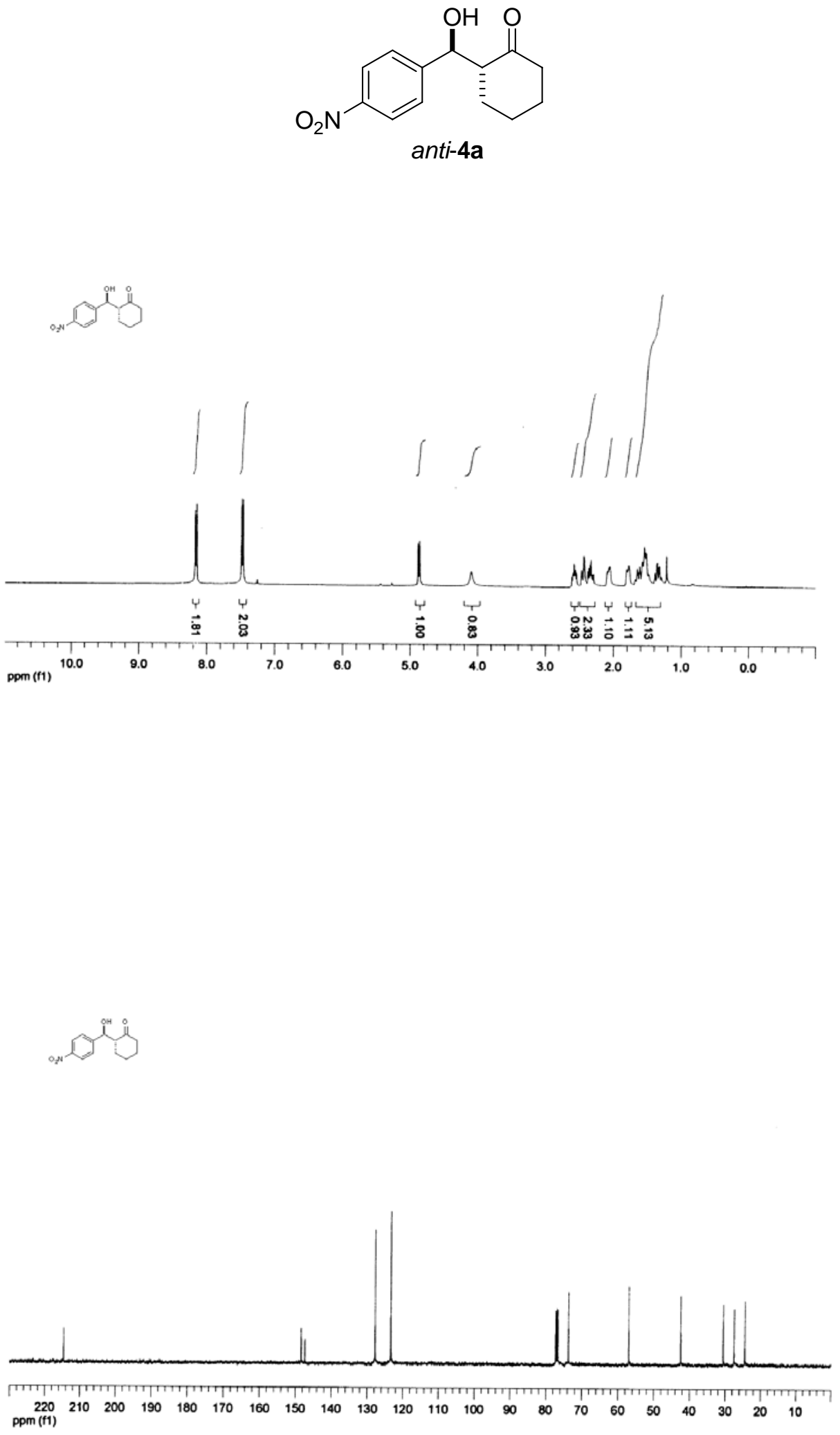

S16 
<smiles>O=C1CCCCC1C(O)c1ccccc1[N+](=O)[O-]</smiles>

syn-4b

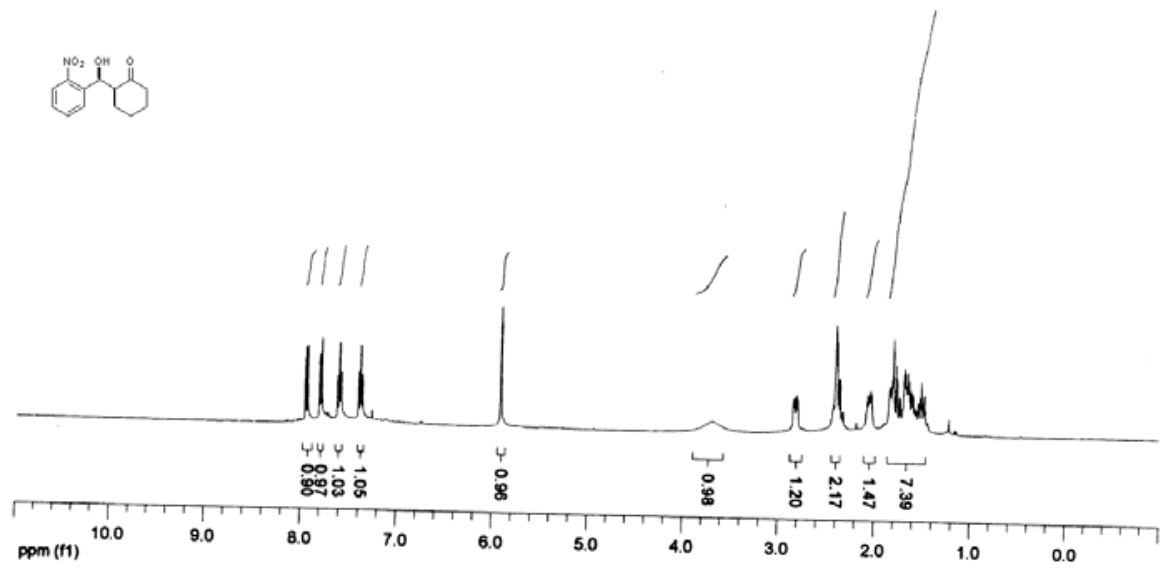

not on

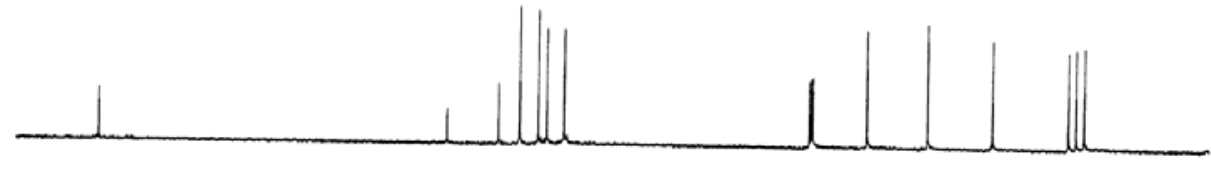
$\begin{array}{clllllllllllllllllllll}220 & 210 & 200 & 190 & 180 & 170 & 160 & 150 & 140 & 130 & 120 & 110 & 100 & 90 & 80 & 70 & 60 & 50 & 40 & 30 & 20 & 10\end{array}$ 
<smiles>O=C1CCCC[C@H]1C(O)c1ccccc1[N+](=O)[O-]</smiles>

anti-4b

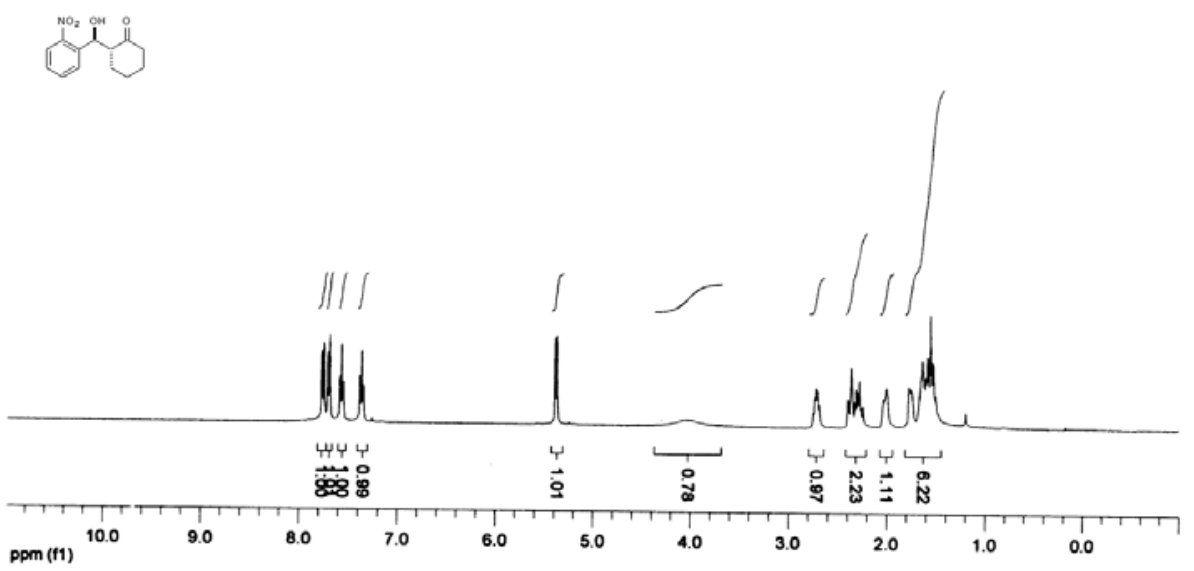

110

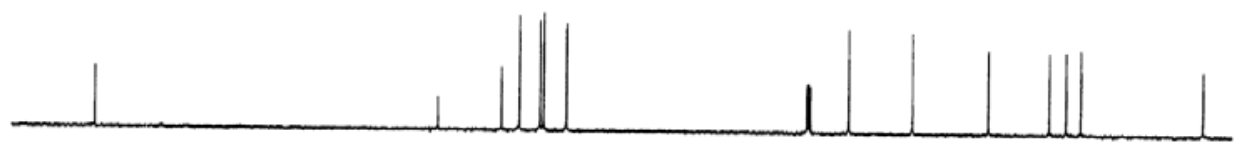

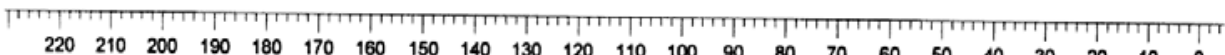
$\begin{array}{rllllllllllllllllllllll}220 & 210 & 200 & 190 & 180 & 170 & 160 & 150 & 140 & 130 & 120 & 110 & 100 & 90 & 80 & 70 & 60 & 50 & 40 & 30 & 20 & 10 & 0 \\ \operatorname{ppm}(\mathrm{f1}) & & & & & & & \end{array}$ 

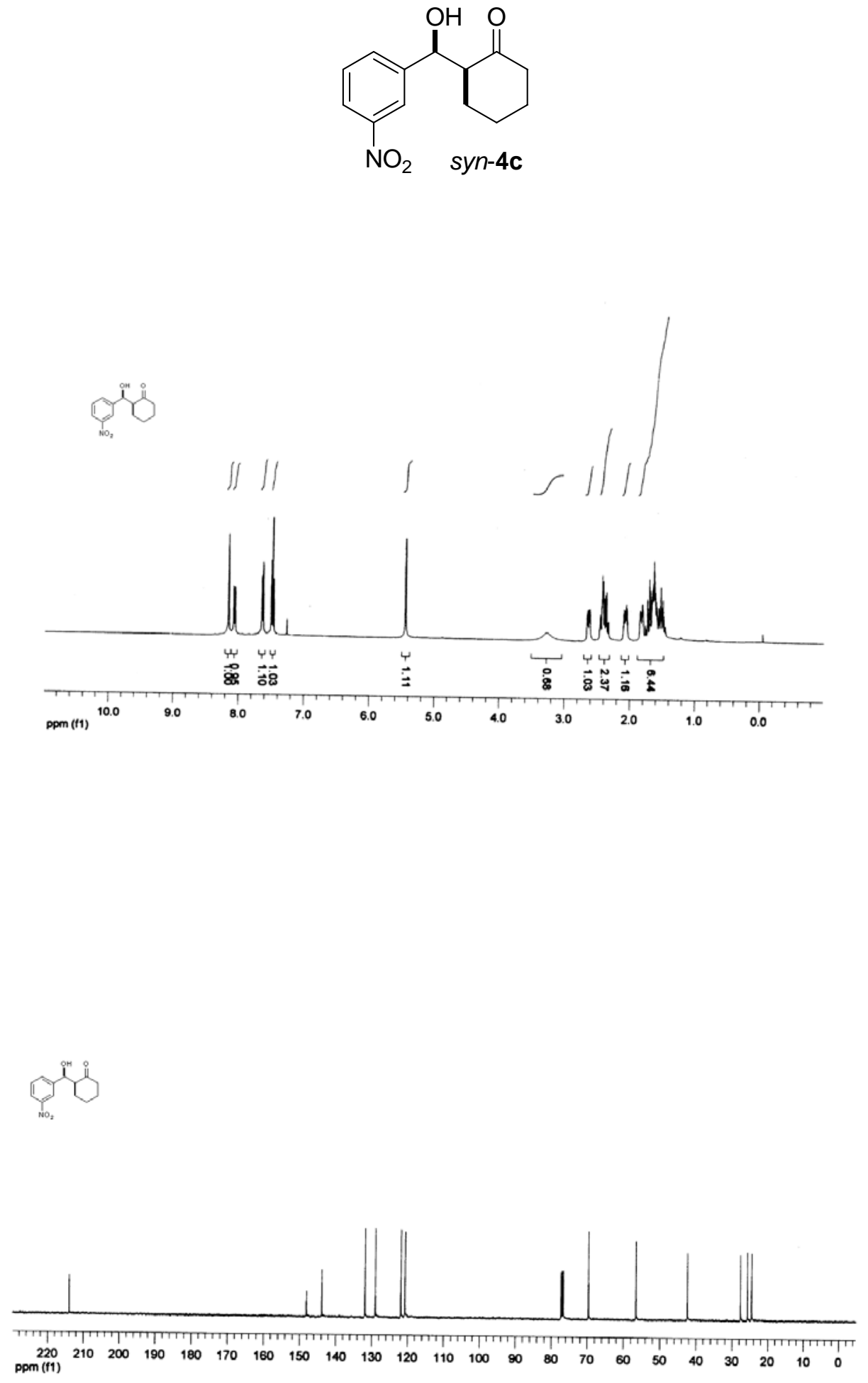

S19 


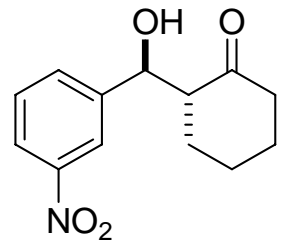

anti-4c

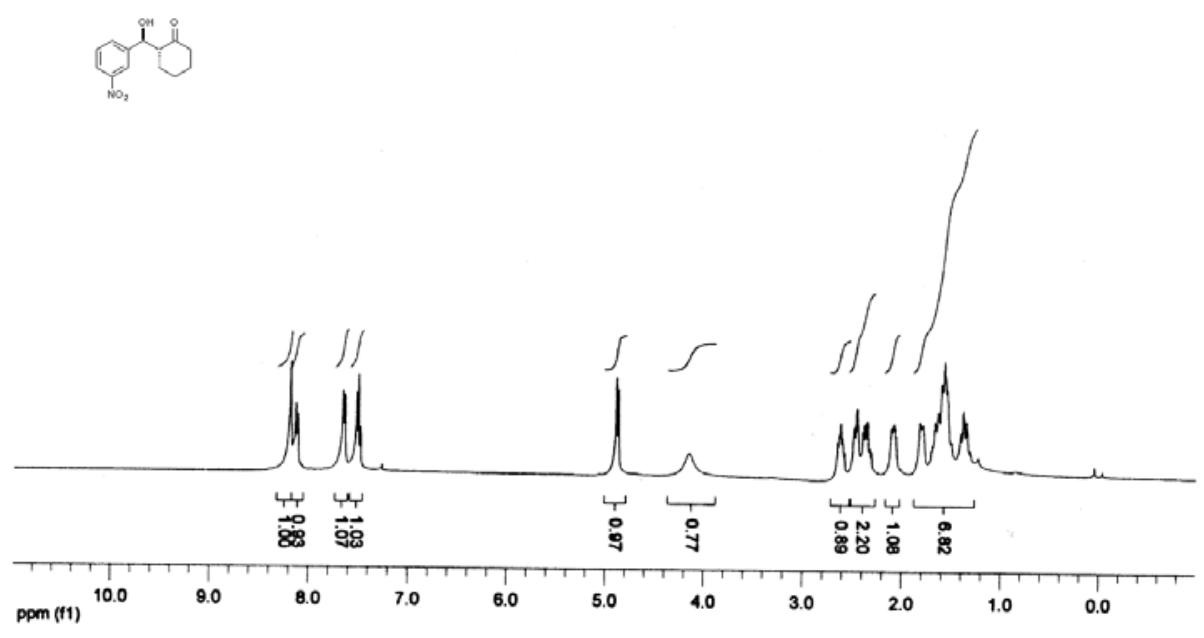

$\underbrace{i n}_{n, 2}$

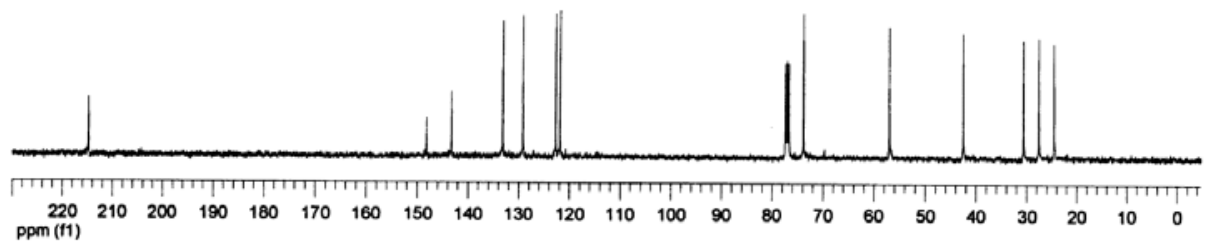


<smiles>N#Cc1ccc(C(O)C2CCCCC2=O)cc1</smiles>
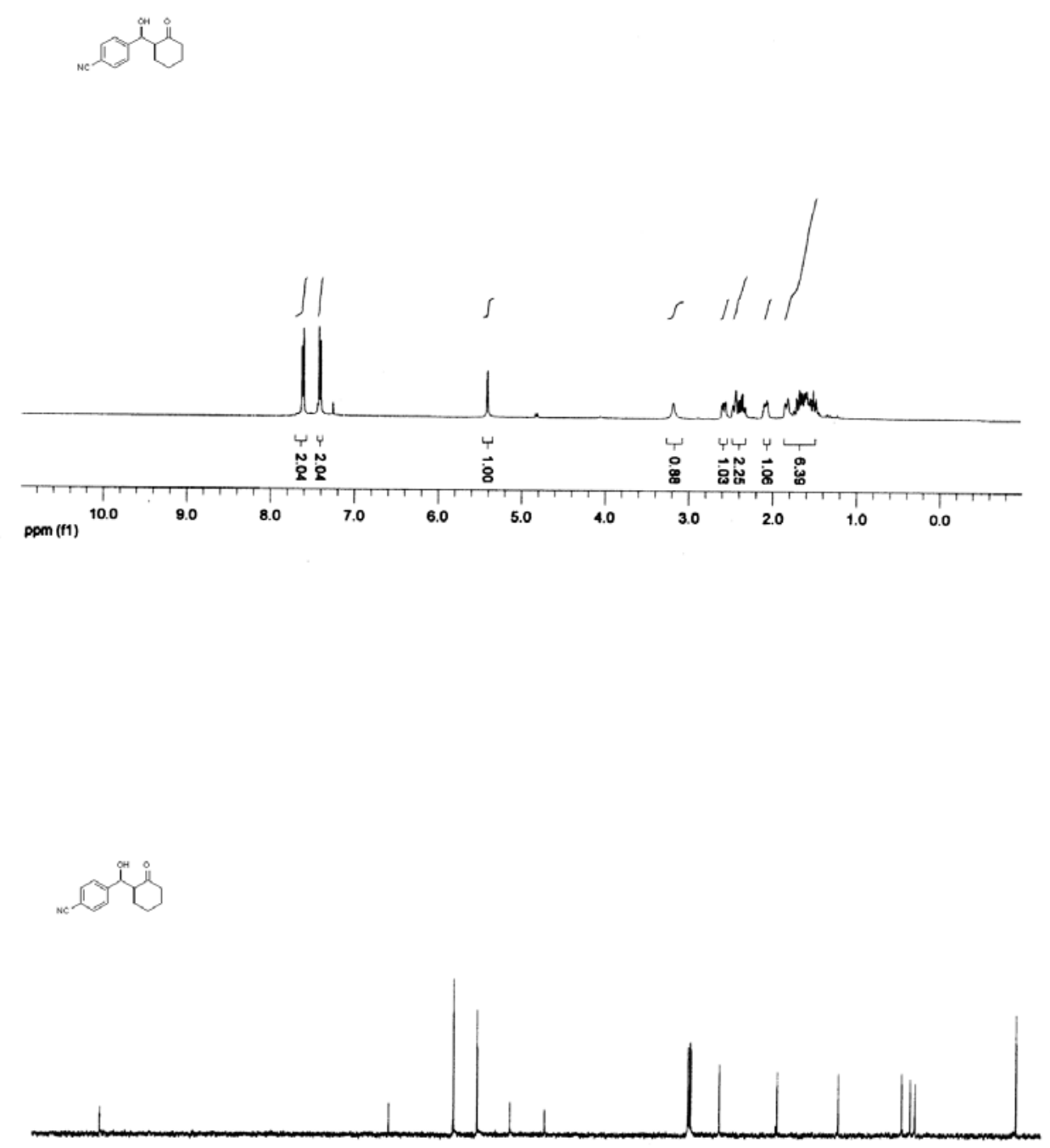

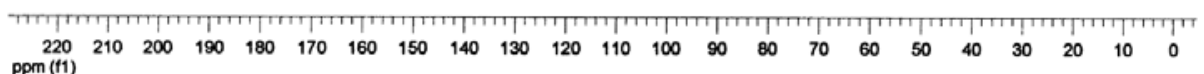




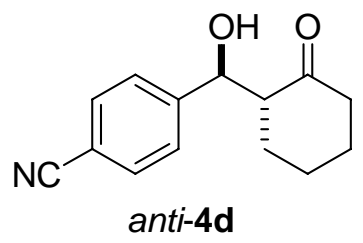

. .
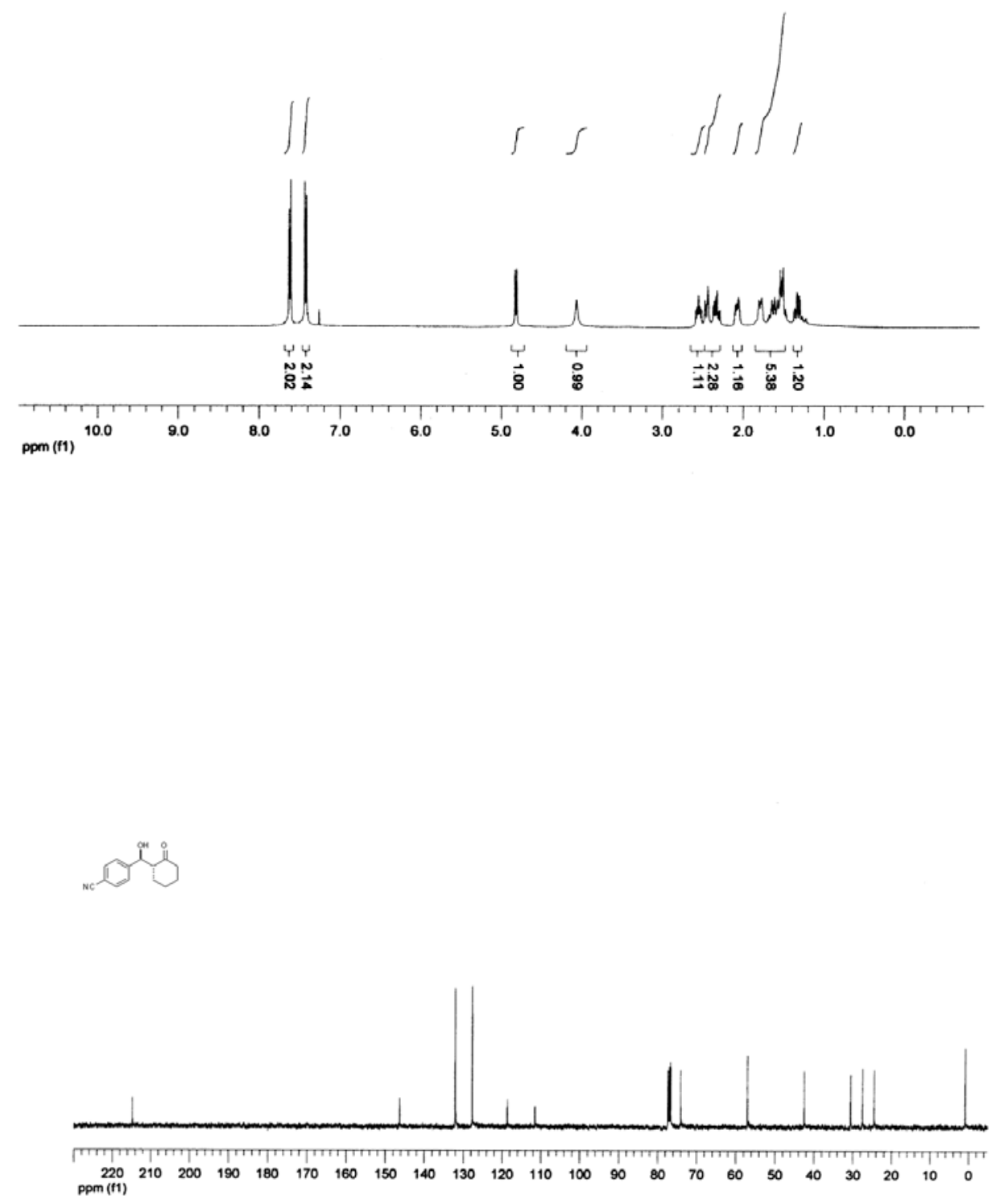

S22 
<smiles>O=C1CCCCC1C(O)c1ccc(Cl)cc1</smiles>
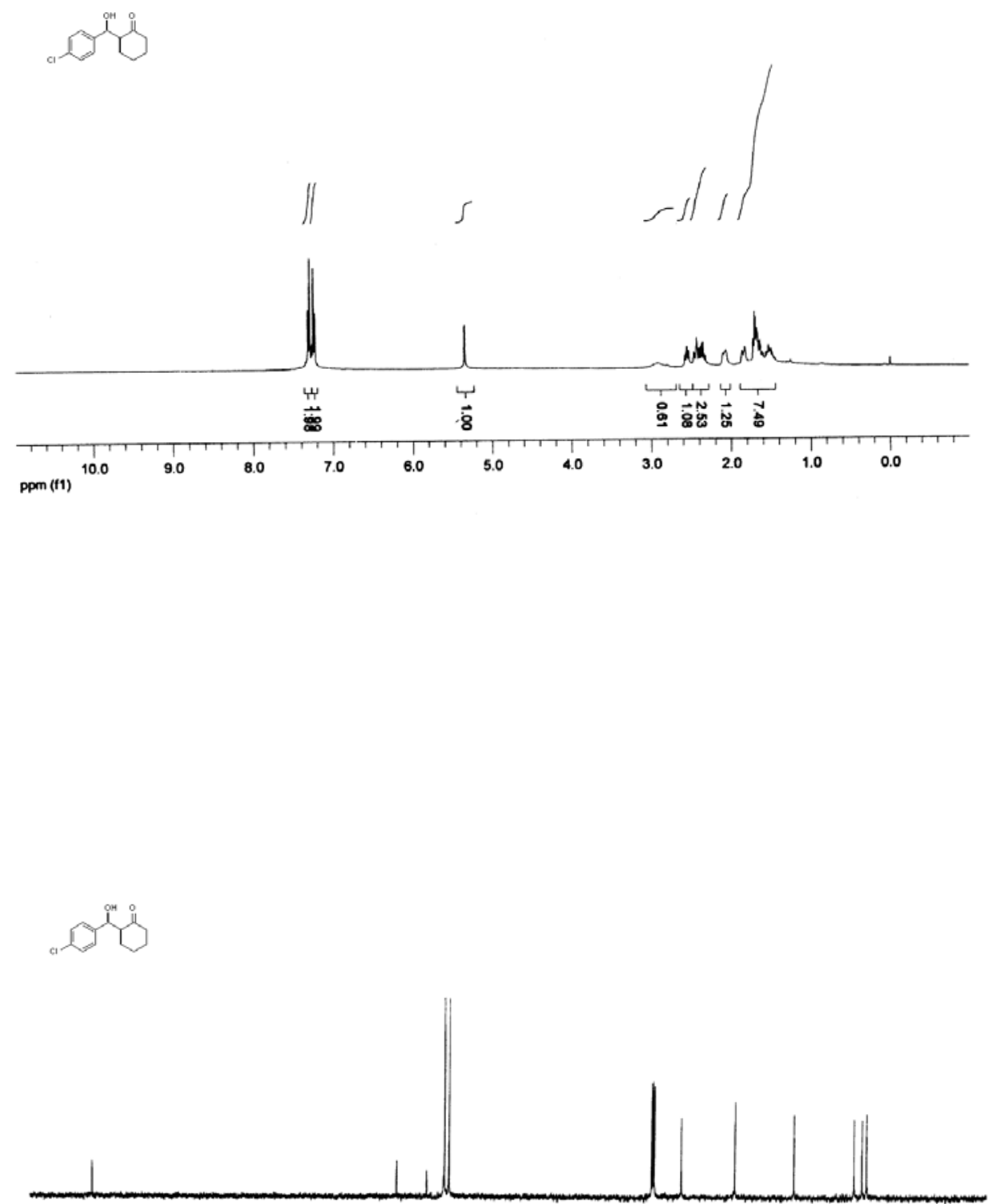
\begin{tabular}{lllllllllllllllllll|l|l|l|l|l|l|l|l|l|}
\hline 220 & 210 & 200 & 190 & 180 & 170 & 160 & 150 & 140 & 130 & 120 & 110 & 100 & 90 & 80 & 70 & 60 & 50 & 40 & 30 & 20 & 10 & 0
\end{tabular} 
<smiles>CC(C)(C)C(O)c1ccc(Cl)cc1</smiles>
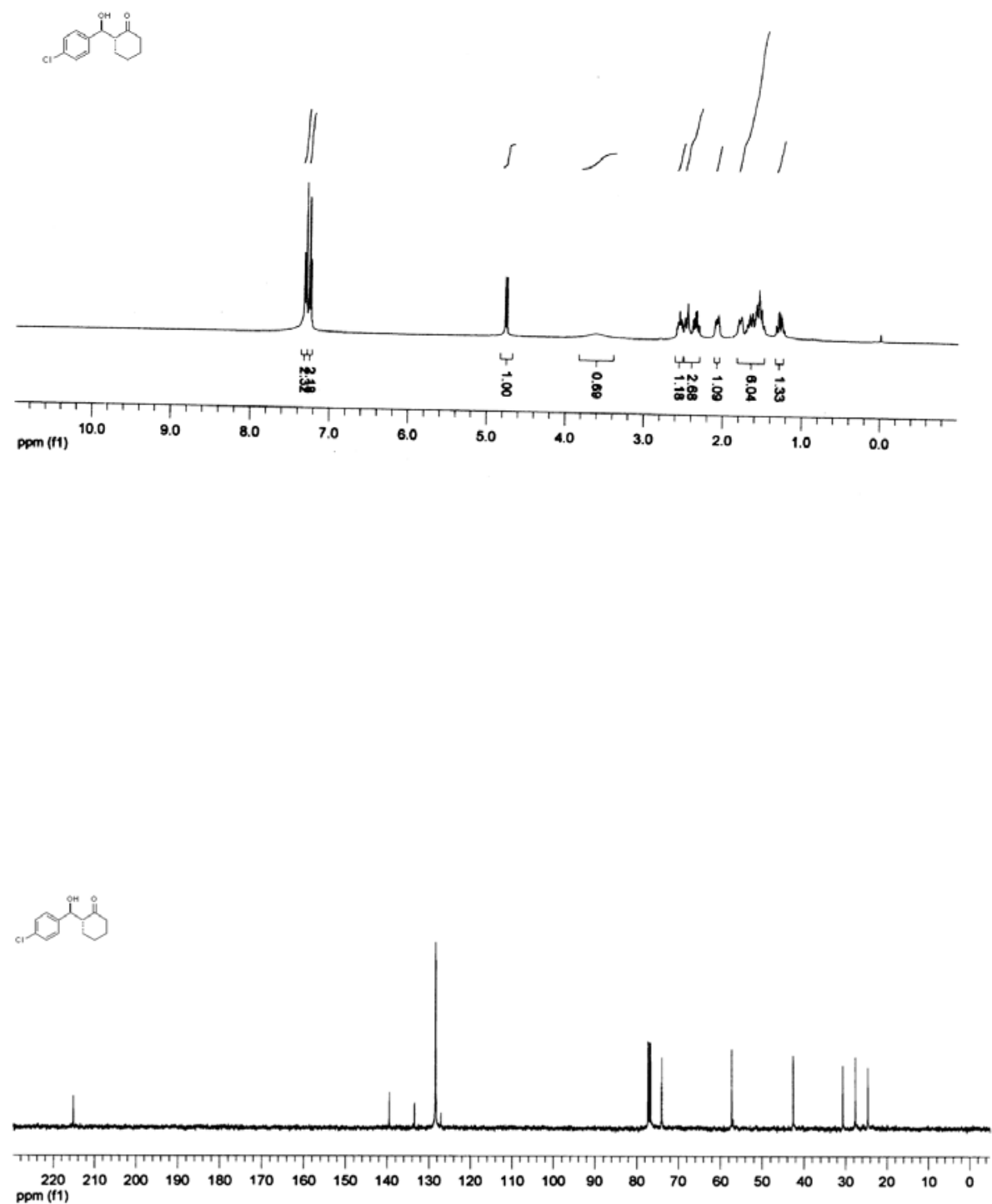
<smiles>O=C1CCCCC1C(O)c1ccccc1Cl</smiles>

둔다

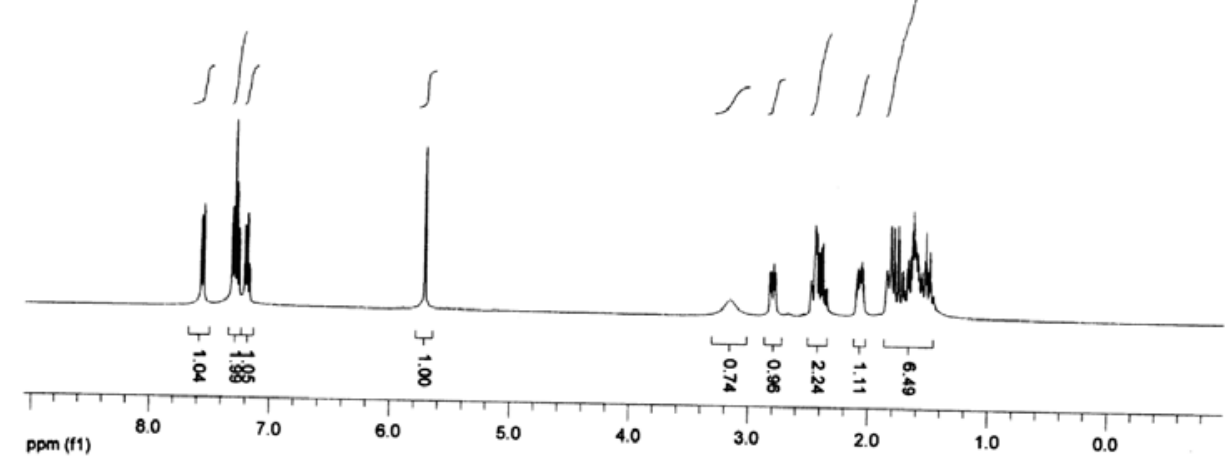

ors

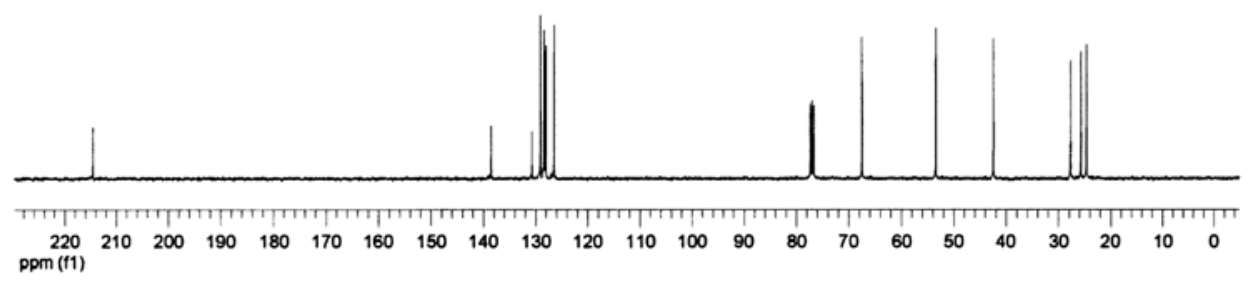

S25 

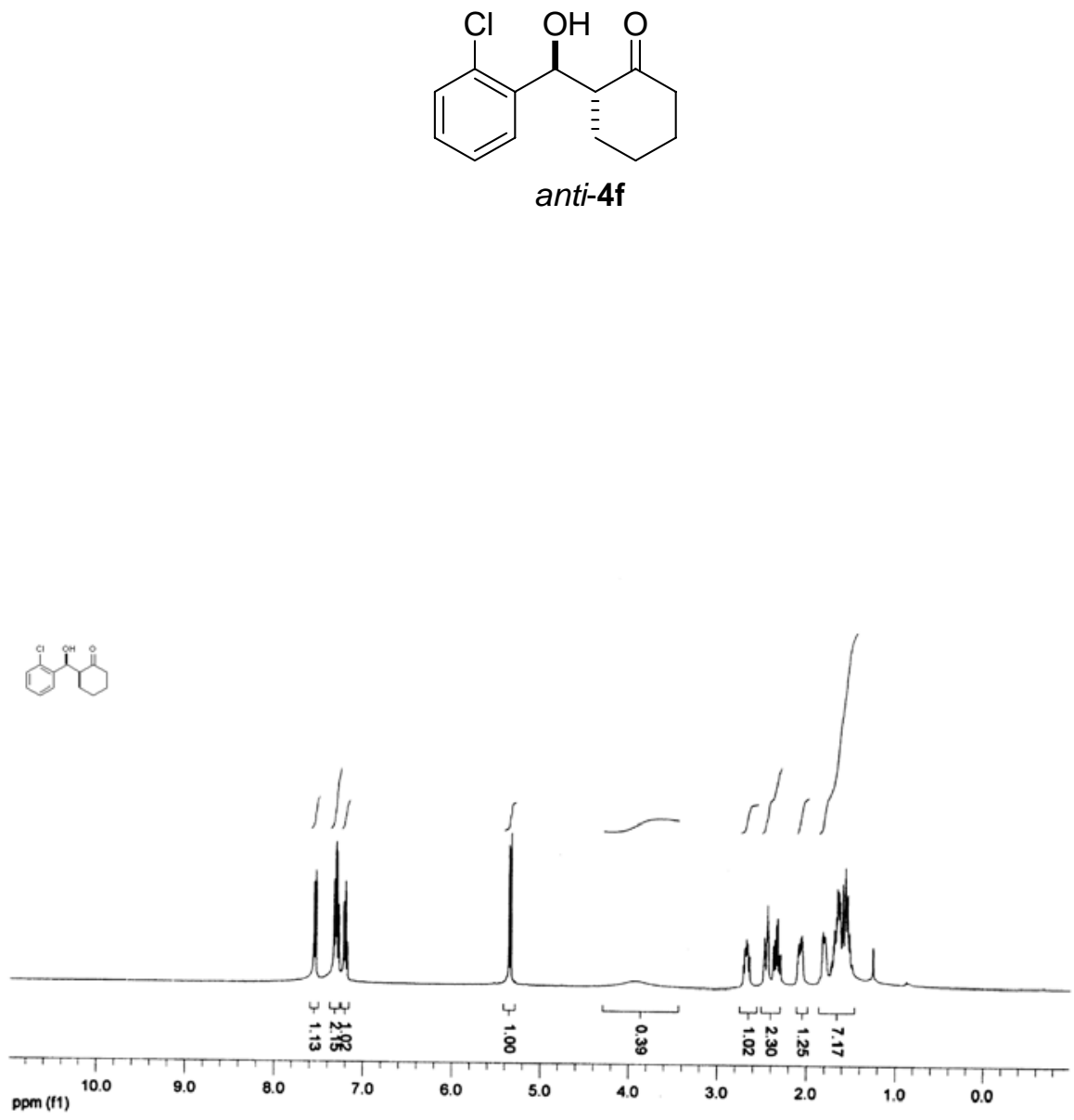

sos

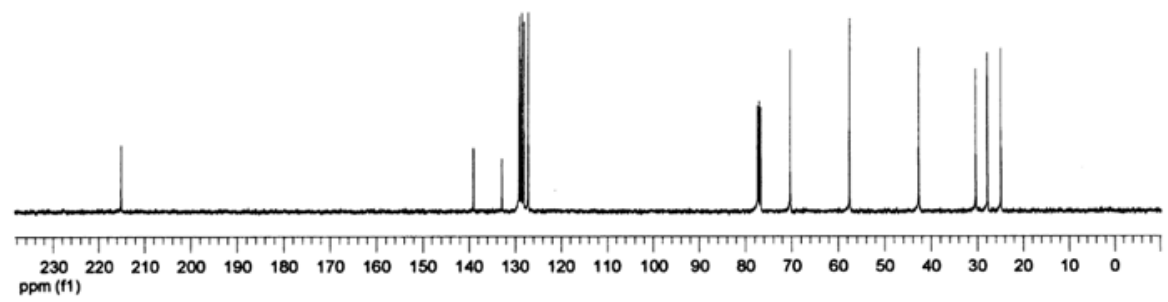

S26 
<smiles>O=C1CCCCC1C(O)c1ccc(Br)cc1</smiles>
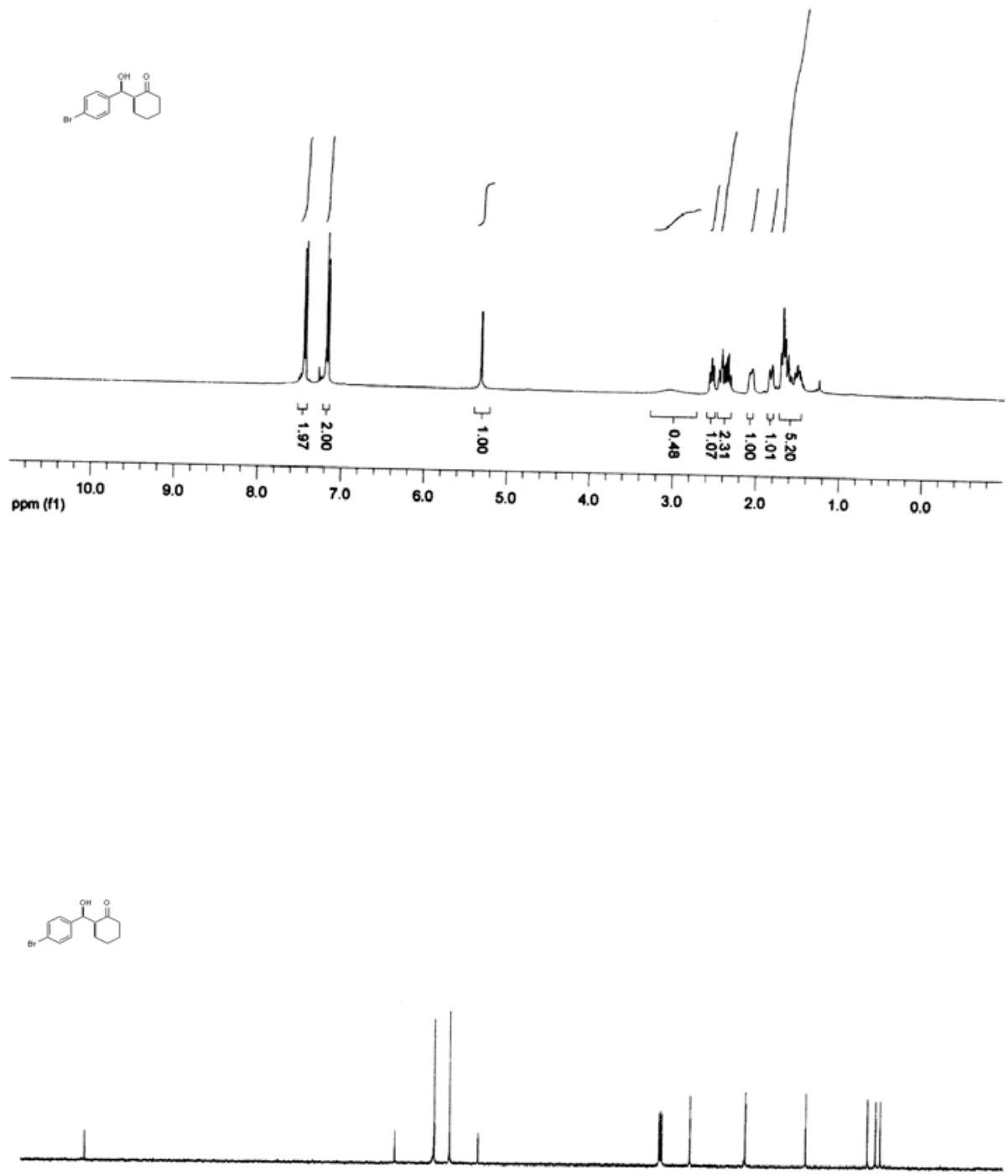

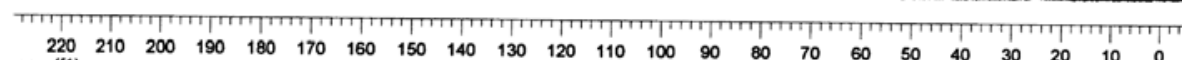

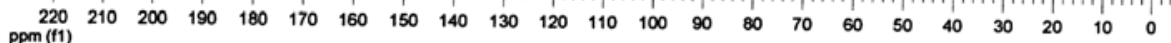


<smiles>O=C1CCCCC1C(O)c1ccc(Br)cc1</smiles>
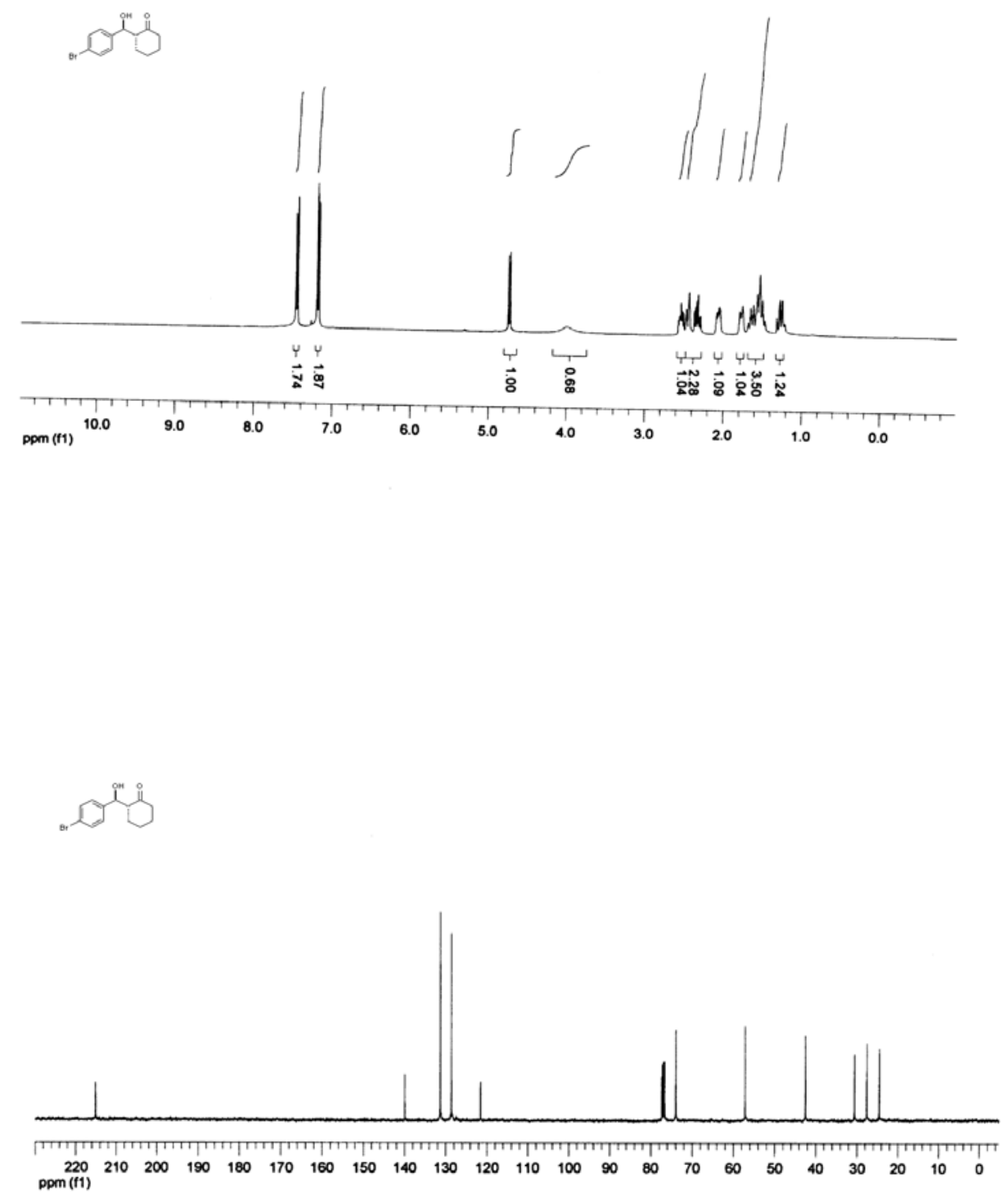
<smiles>O=C1CCCCC1C(O)c1ccccc1</smiles>

syn-4h

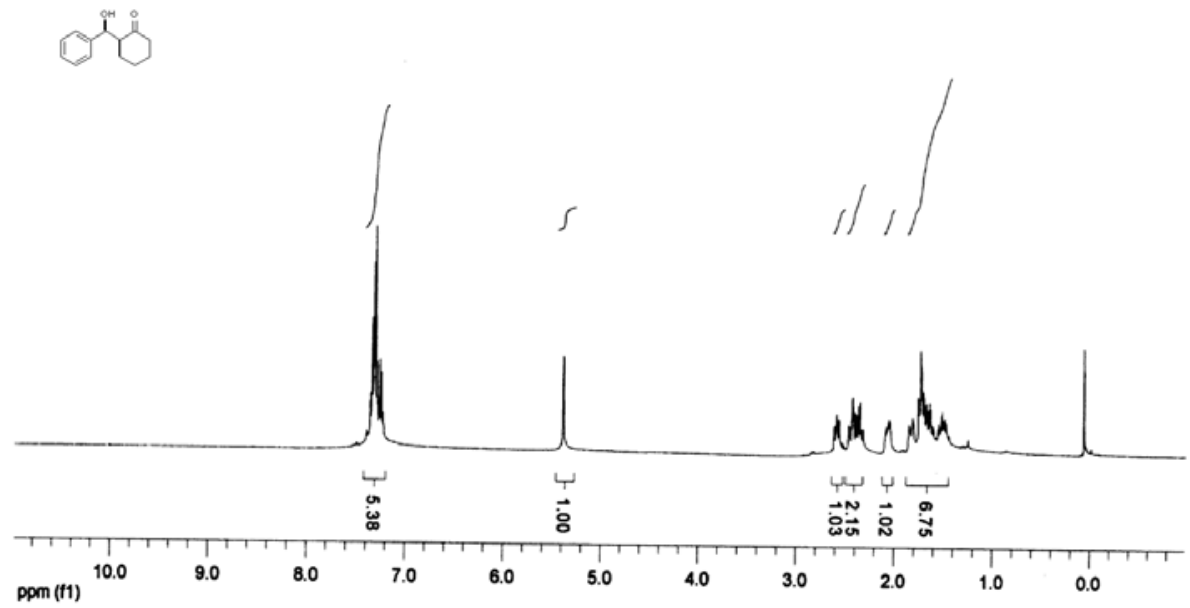

cin

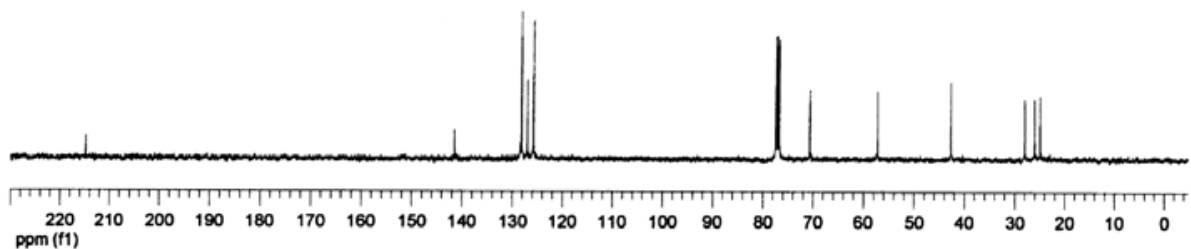


<smiles>O=C1CCCC[C@H]1C(O)c1ccccc1</smiles>

anti-4h

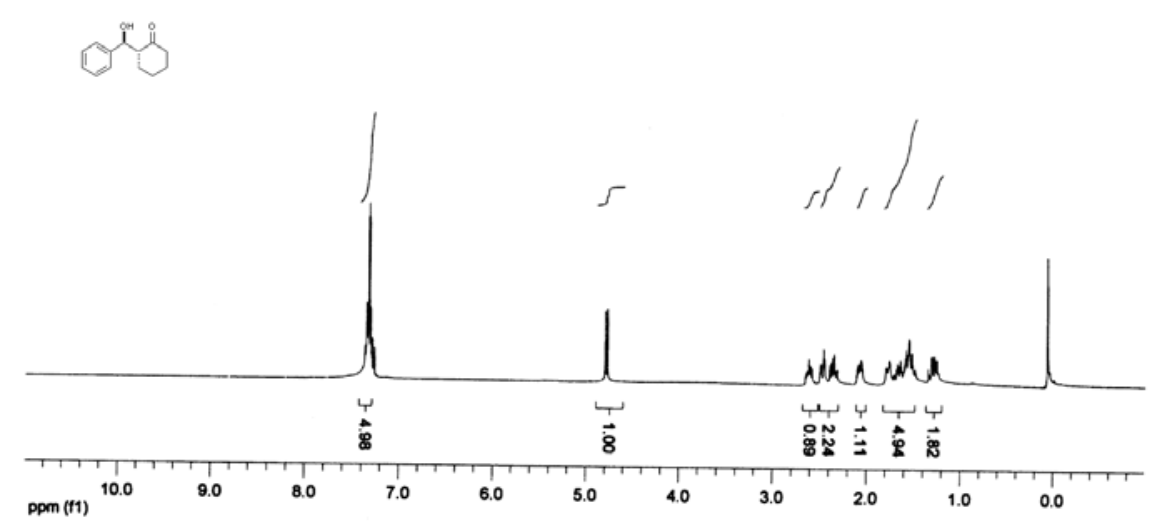

(1)

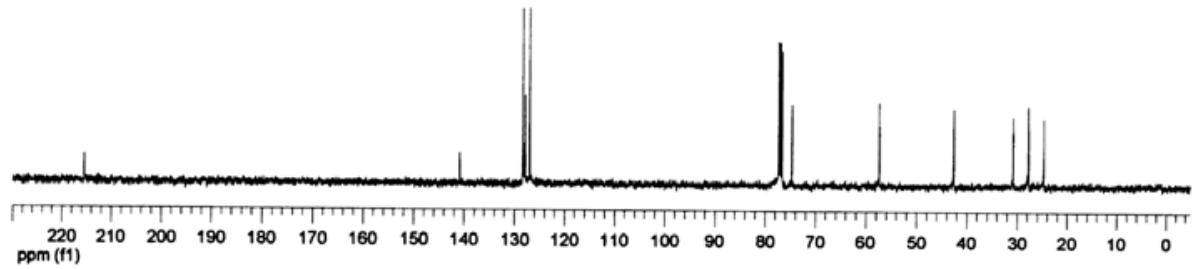


<smiles>O=C1CCCC[C@H]1C(O)c1cccc2ccccc12</smiles>

anti-4i
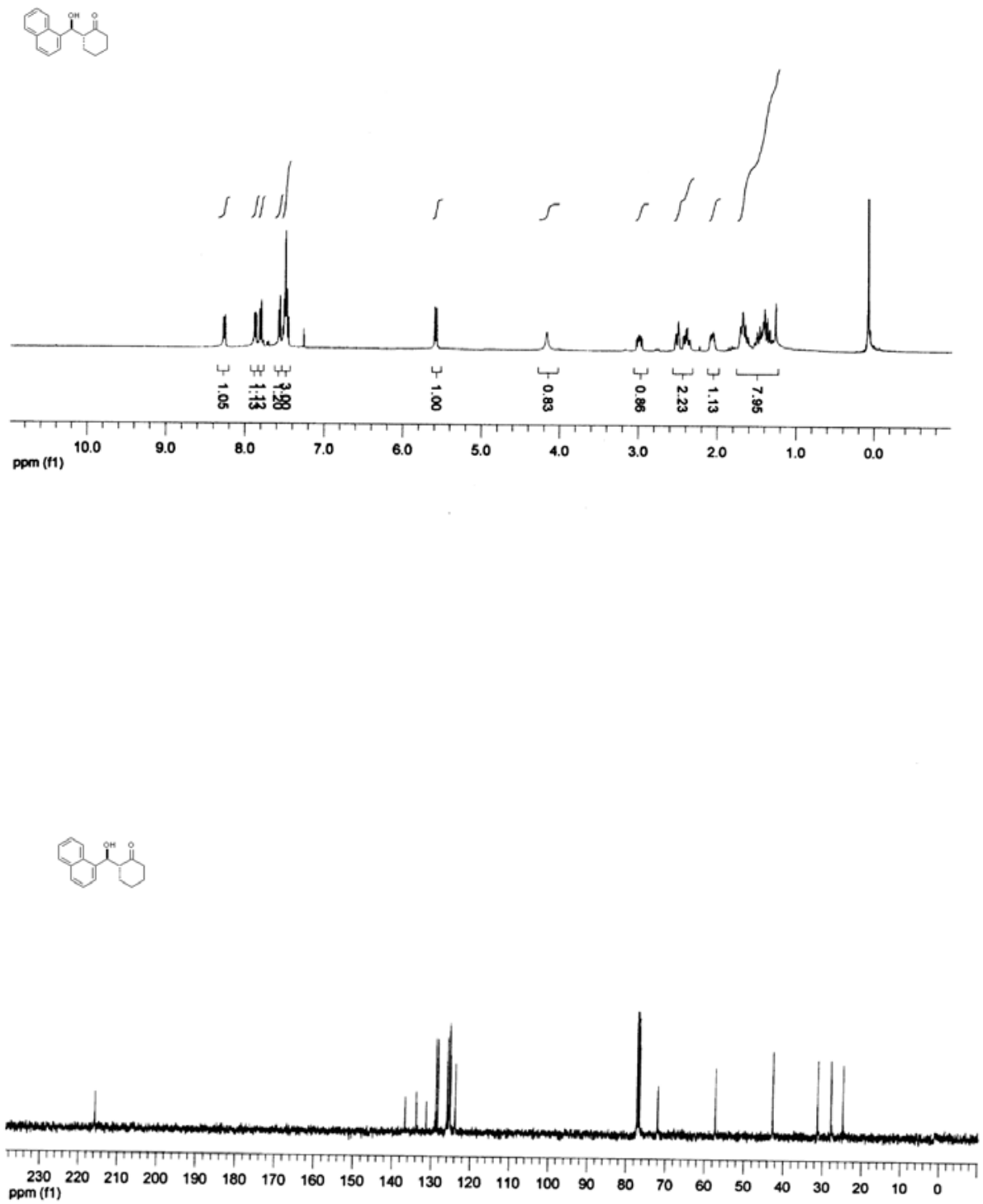


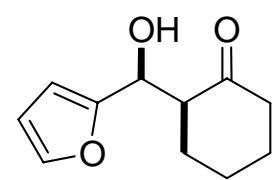

syn-4j
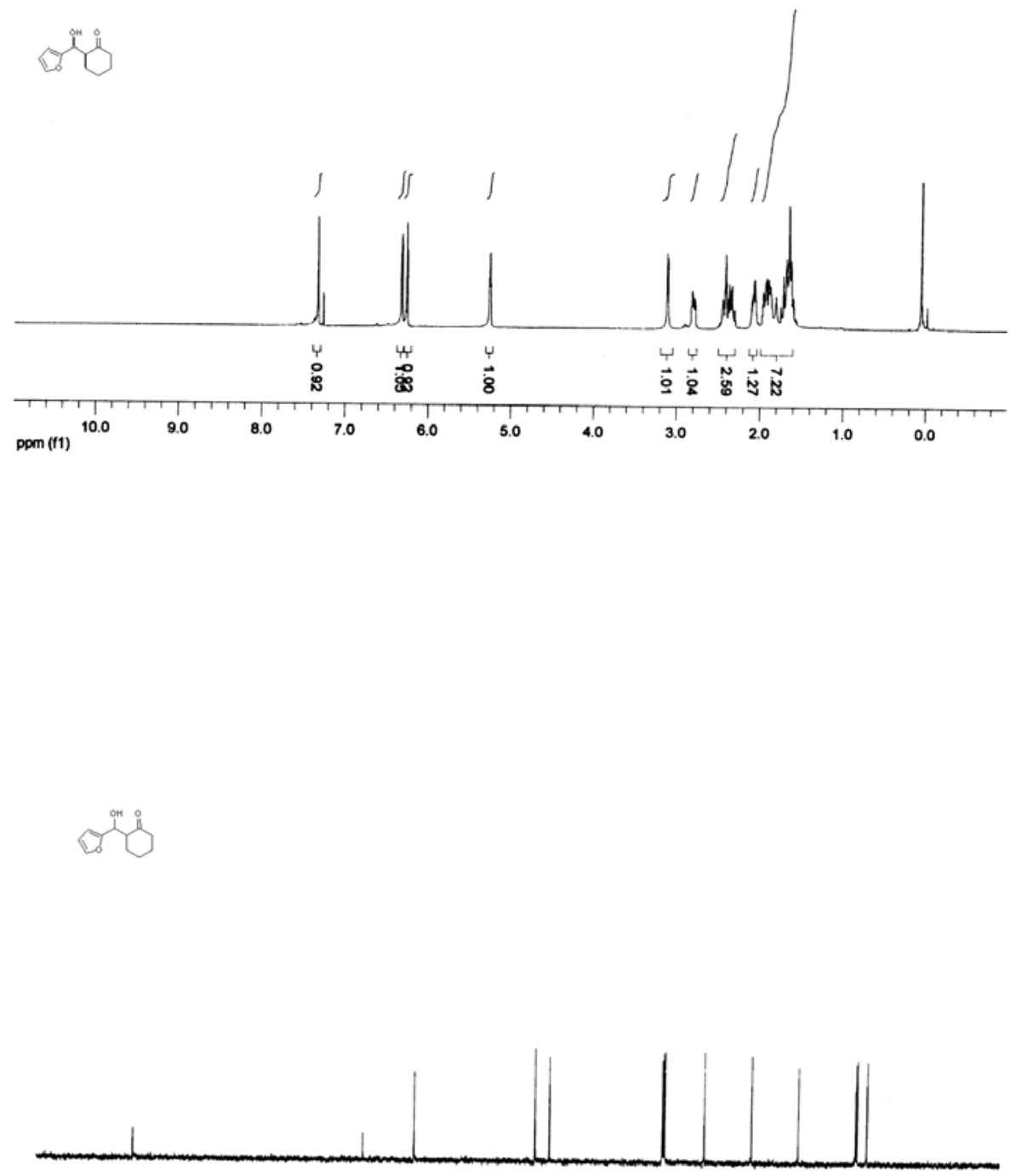

$\begin{aligned} & 230 \\ & \text { ppm(f1) }\end{aligned} 20 \begin{array}{lllllllllllllllllllllll} & 210 & 200 & 190 & 180 & 170 & 160 & 150 & 140 & 130 & 120 & 110 & 100 & 90 & 80 & 70 & 60 & 50 & 40 & 30 & 20 & 10 & 0\end{array}$ 
<smiles>O=C1CCCC[C@H]1C(O)c1ccco1</smiles>

anti-4j
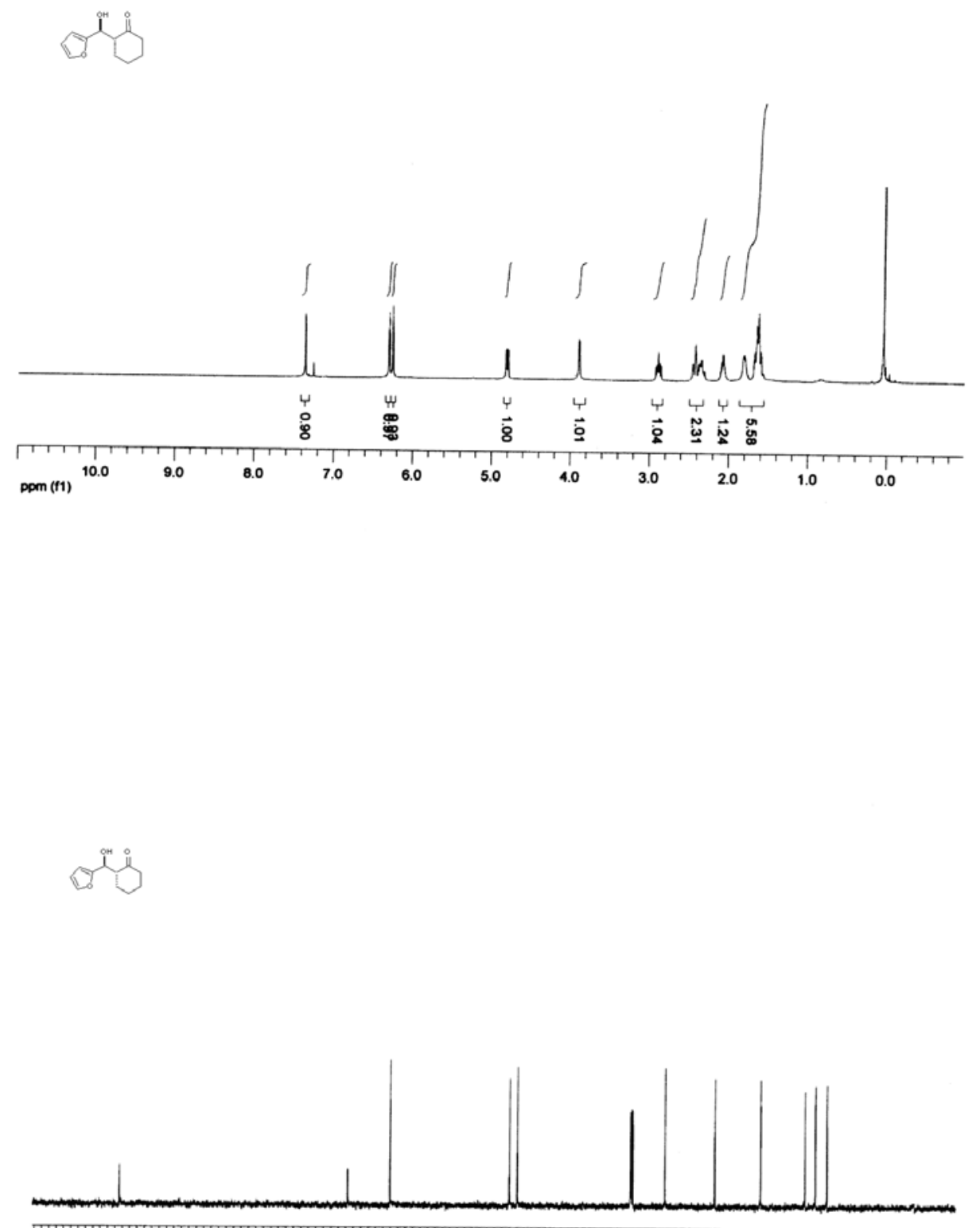

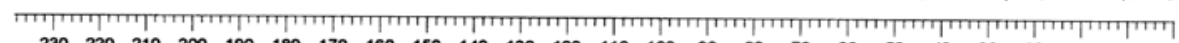

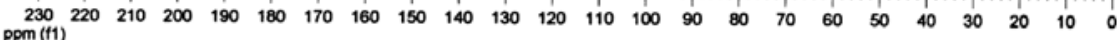


<smiles>Cc1ccc(C(O)C2CCCCC2=O)cc1</smiles>

syn-4k

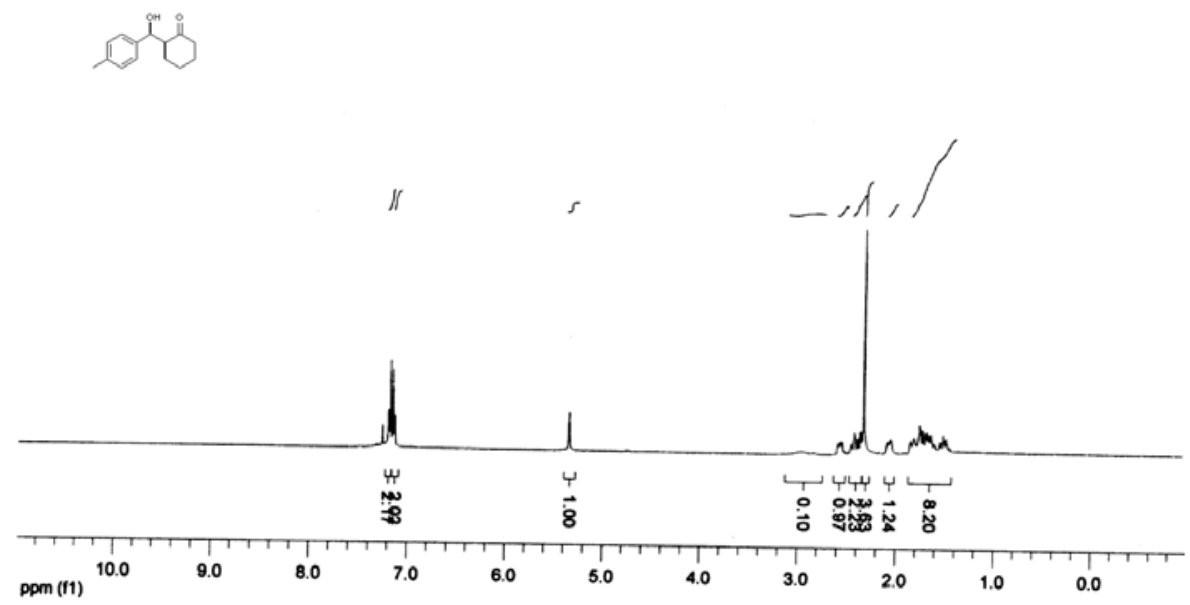

JR222AC-methyl-syn
050616

舟茴

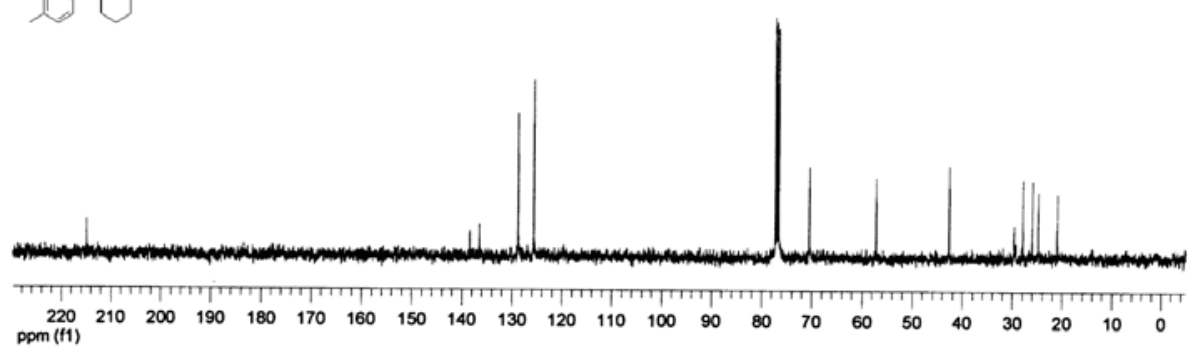


<smiles>Cc1ccc(C(O)[C@H]2CCCCC2=O)cc1</smiles>

anti-4k
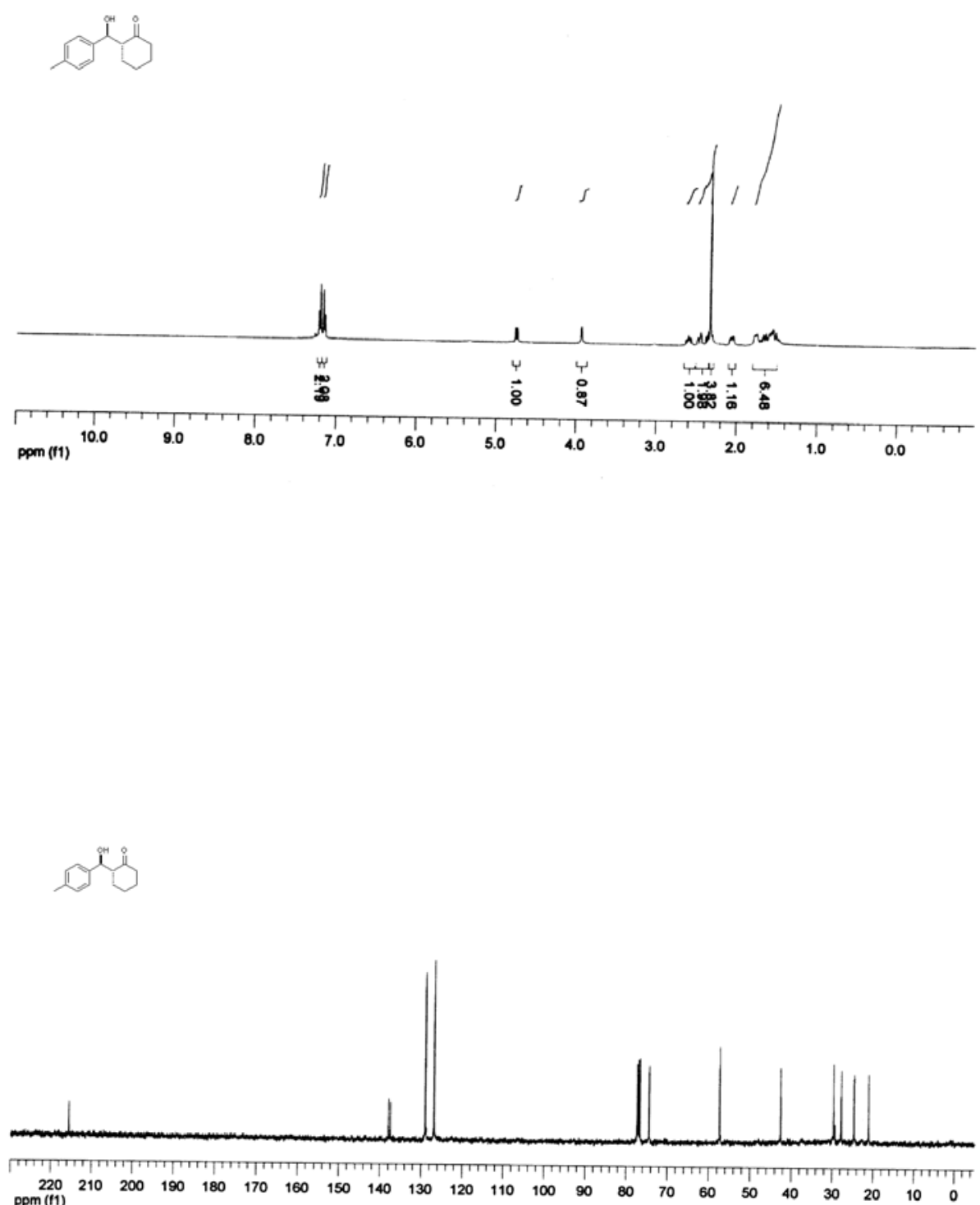
<smiles>CC(=O)[C@H](C)C(O)c1ccc([N+](=O)[O-])cc1</smiles>

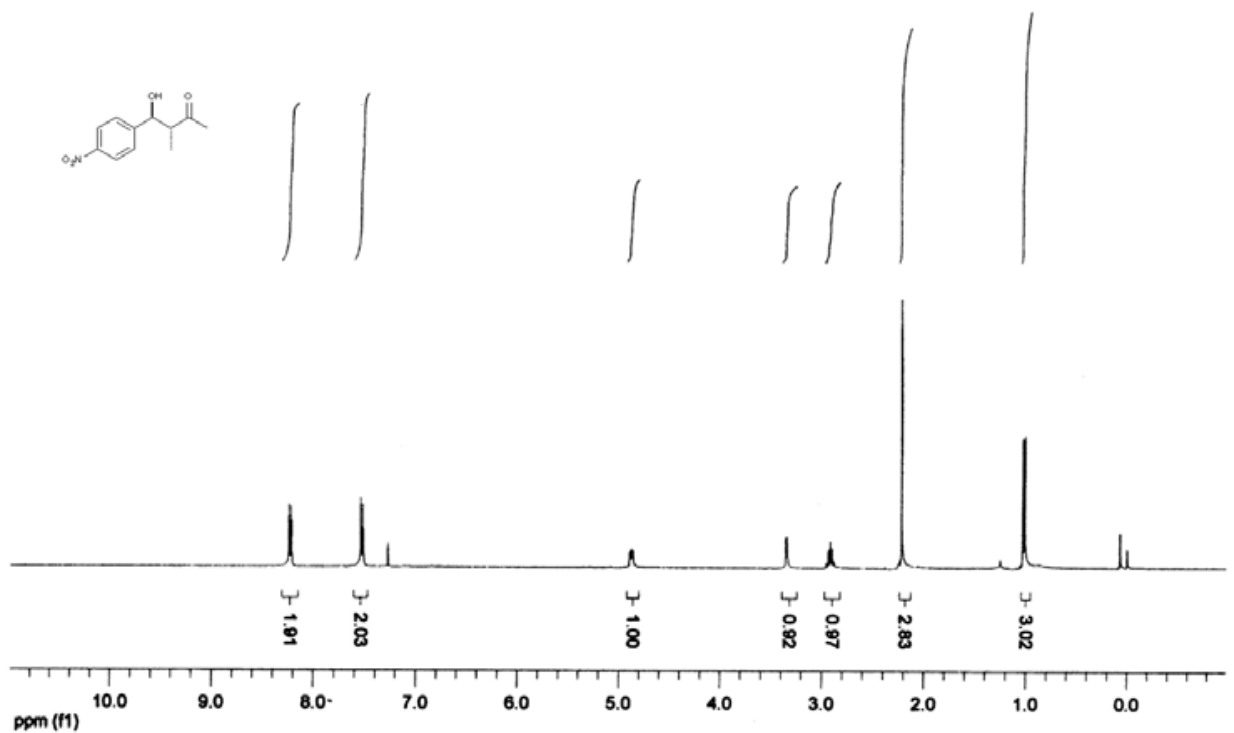

, 1$)^{n ! n}$

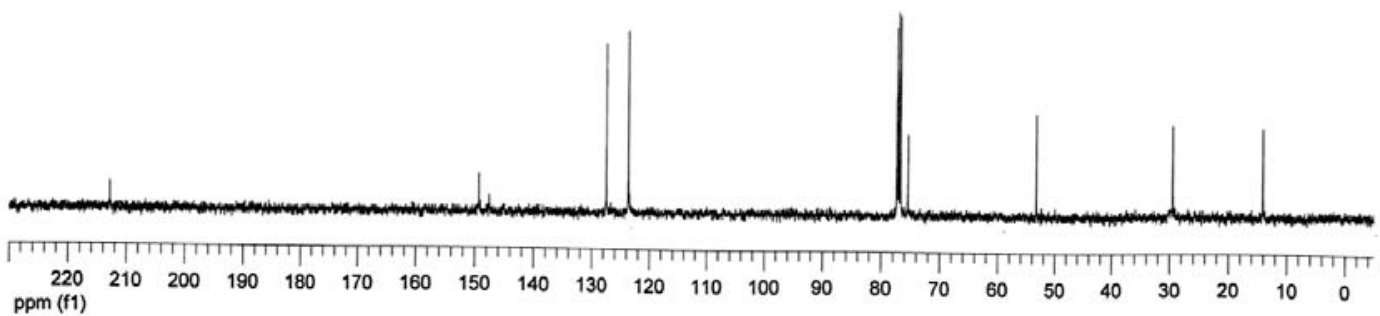




\section{HPLC Spectra}

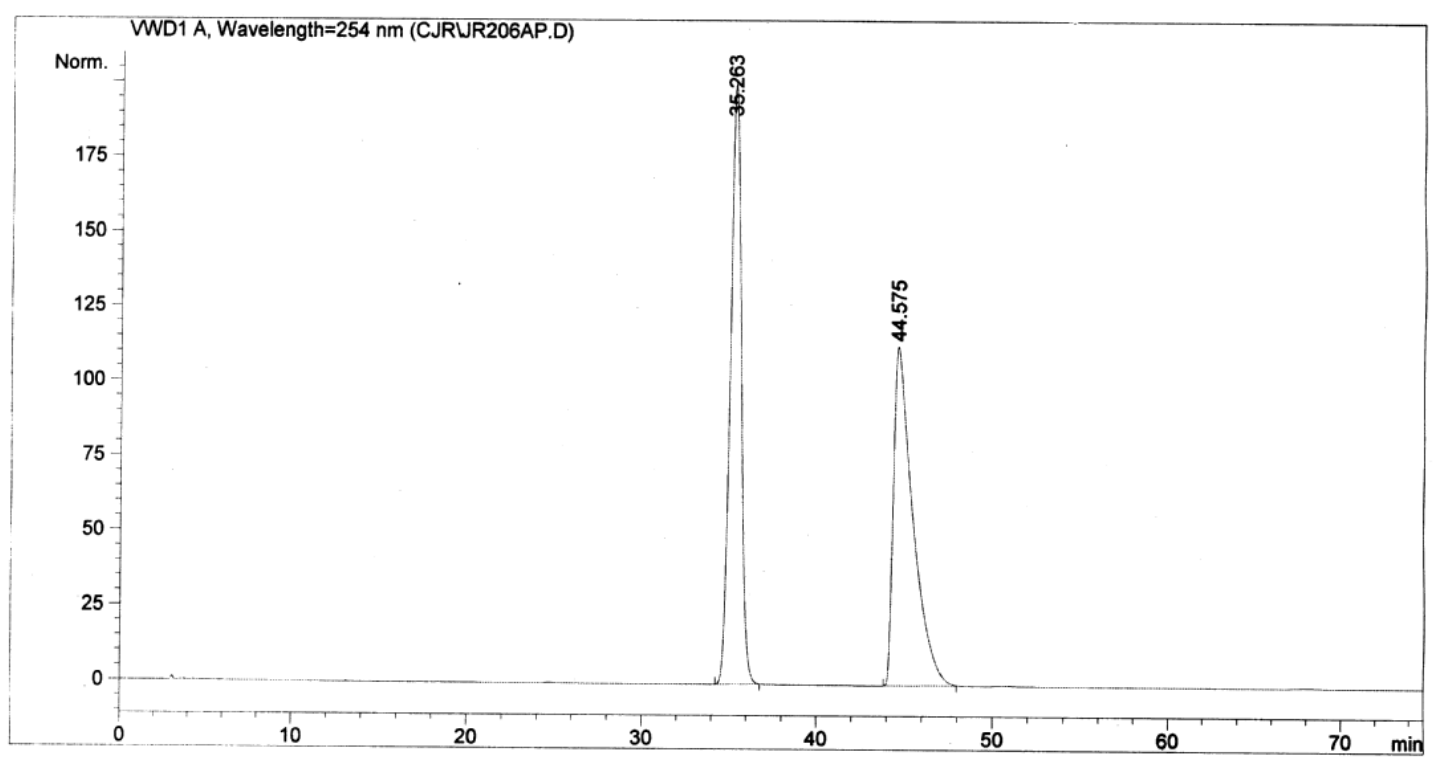<smiles>O=C1CCCCC1C(O)c1ccc([N+](=O)[O-])cc1</smiles>

racemic-syn-4a

Signal 1: VWD1 A, Wavelength=254 nm

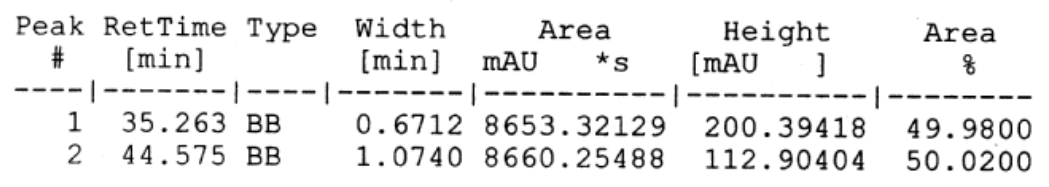

Totals :

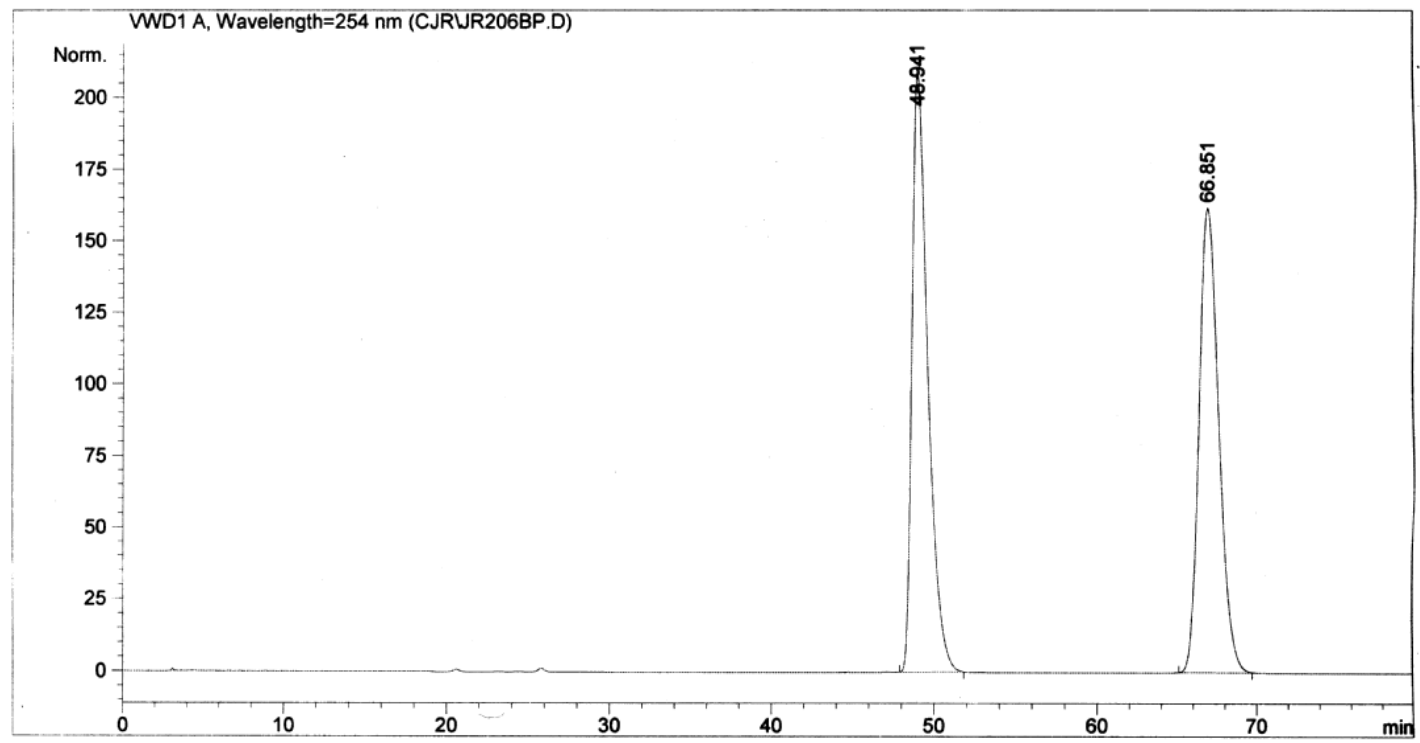<smiles>O=C1CCCCC1C(O)c1ccc([N+](=O)[O-])cc1</smiles>

racemic-anti-4a

Signal 1: VWD1 A, Wavelength=254 nm

\begin{tabular}{|c|c|c|c|c|c|c|}
\hline $\begin{array}{c}\text { Peak } \\
\#\end{array}$ & $\begin{array}{l}\text { RetTime } \\
\text { [min] }\end{array}$ & Type & $\begin{array}{l}\text { Width } \\
\text { [min] }\end{array}$ & 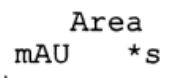 & $\begin{array}{l}\text { Height } \\
{[\mathrm{mAU}]}\end{array}$ & $\begin{array}{c}\text { Area } \\
8\end{array}$ \\
\hline $\begin{array}{l}1 \\
2\end{array}$ & $\begin{array}{l}48.941 \\
66.851\end{array}$ & $\begin{array}{l}\text { BB } \\
\text { BB }\end{array}$ & $\begin{array}{l}0.9999 \\
1.3222\end{array}$ & $\begin{array}{l}1.40445 \mathrm{e} 4 \\
1.40234 \mathrm{e} 4\end{array}$ & $\begin{array}{l}208.49153 \\
162.11780\end{array}$ & $\begin{array}{l}50.0375 \\
49.9625\end{array}$ \\
\hline Total & Is : & & & $2.80679 \mathrm{e} 4$ & 370.60933 & \\
\hline
\end{tabular}



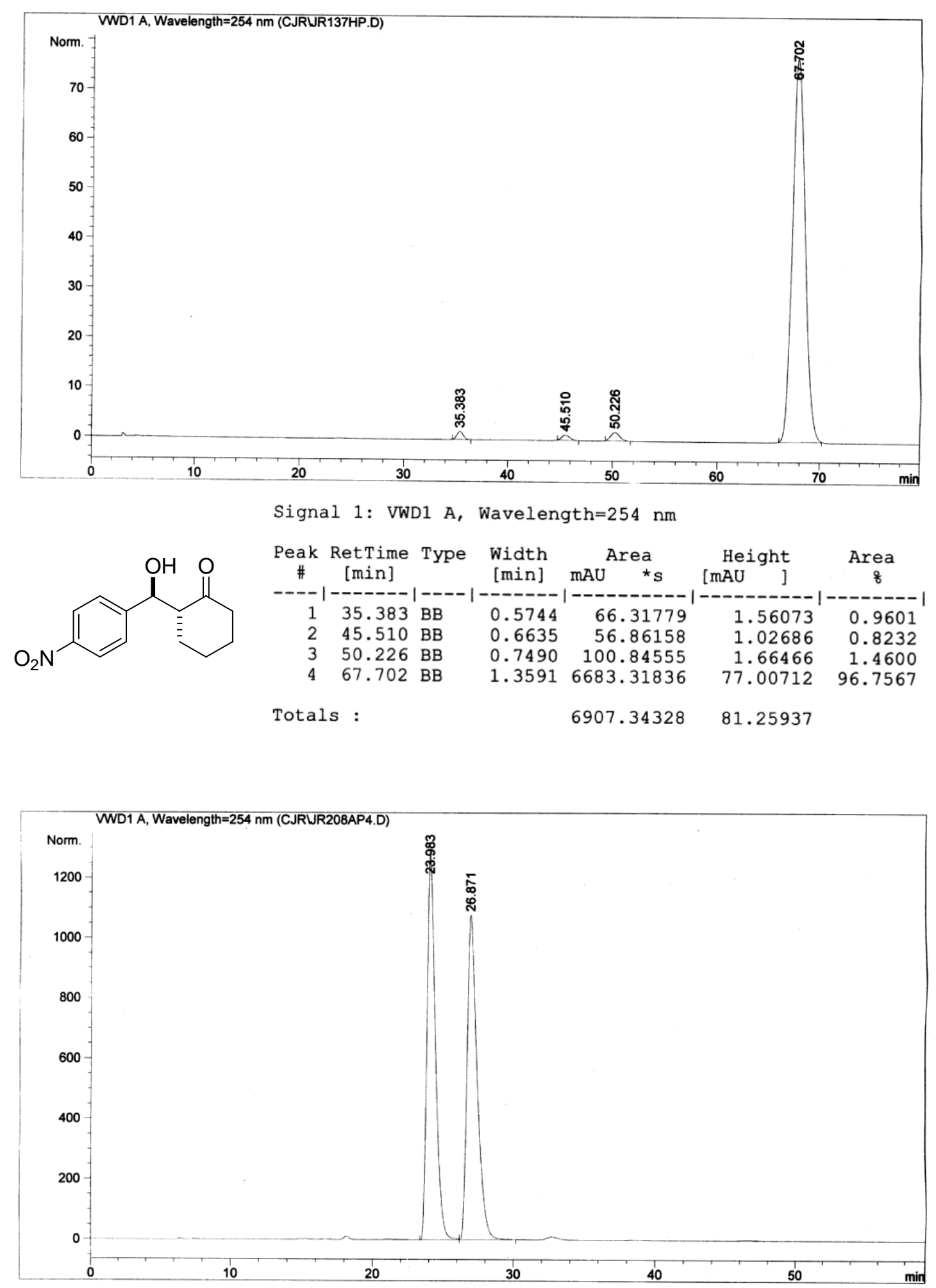<smiles>O=C1CCCCC1C(O)c1ccccc1[N+](=O)[O-]</smiles>

racemic-syn-4b

Signal 1: VWD1 A, Wavelength=254 nm

$$
\begin{aligned}
& \text { Peak RetTime Type Width Area Height Area }
\end{aligned}
$$

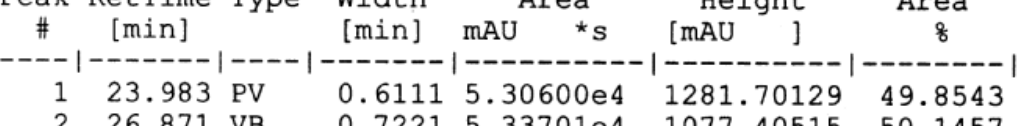

$$
\begin{aligned}
& \begin{array}{llllll}
2 & 26.871 \text { VB } & 0.7221 & 5.33701 \mathrm{e} 4 & 1077.40515 & 50.1457
\end{array} \\
& \text { Totals : } \quad 1.06430 \mathrm{e} 5 \quad 2359.10645
\end{aligned}
$$



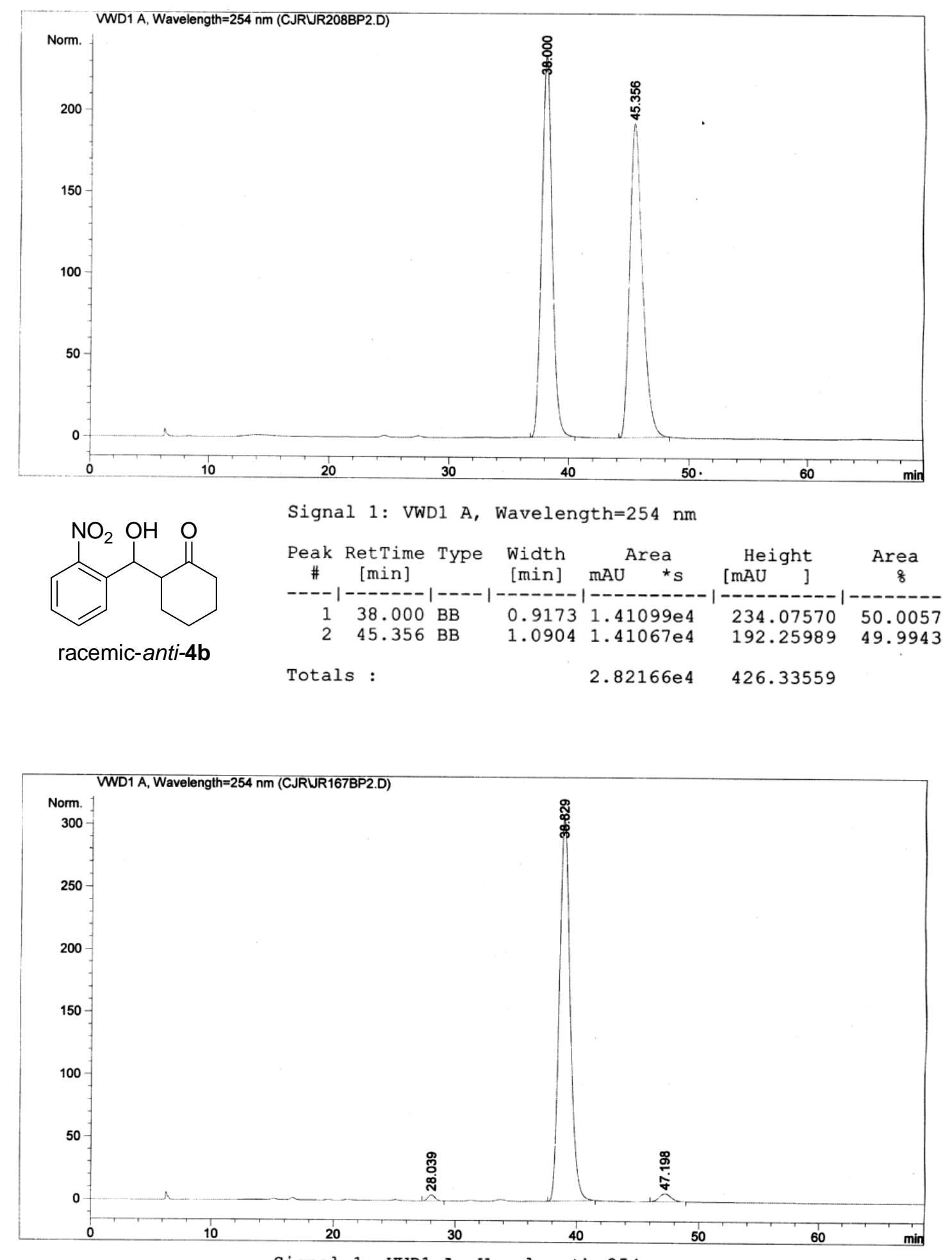

Signal 1: VWD1 A, Wavelength=254 nm<smiles>O=C1CCCC[C@H]1C(O)c1ccccc1[N+](=O)[O-]</smiles>

\begin{tabular}{|c|c|c|c|c|c|c|}
\hline $\begin{array}{c}\text { Peak } \\
\#\end{array}$ & $\begin{array}{c}\text { RetTime } \\
\text { [min] }\end{array}$ & Type & $\begin{array}{l}\text { Width } \\
\text { [min] }\end{array}$ & ${ }_{\mathrm{mAU}}{ }^{\text {Area }}{ }^{*} \mathrm{~s}$ & $\begin{array}{c}\text { Height } \\
{[\mathrm{mAU}]}\end{array}$ & $\begin{array}{c}\text { Area } \\
\text { \& }\end{array}$ \\
\hline $\begin{array}{l}1 \\
2 \\
3\end{array}$ & $\begin{array}{l}28.039 \\
38.829 \\
47.198\end{array}$ & $\begin{array}{l}\mathrm{BB} \\
\mathrm{BB} \\
\mathrm{BB}\end{array}$ & $\begin{array}{l}0.6146 \\
0.9450 \\
0.9762\end{array}$ & $\begin{array}{c}196.92007 \\
1.89174 \mathrm{e} 4 \\
432.59073\end{array}$ & $\begin{array}{r}4.83879 \\
306.21362 \\
6.47342\end{array}$ & $\begin{array}{r}1.0074 \\
96.7795 \\
2.2131\end{array}$ \\
\hline Total & : & & & $1.95469 \mathrm{e} 4$ & 317.52583 & \\
\hline
\end{tabular}




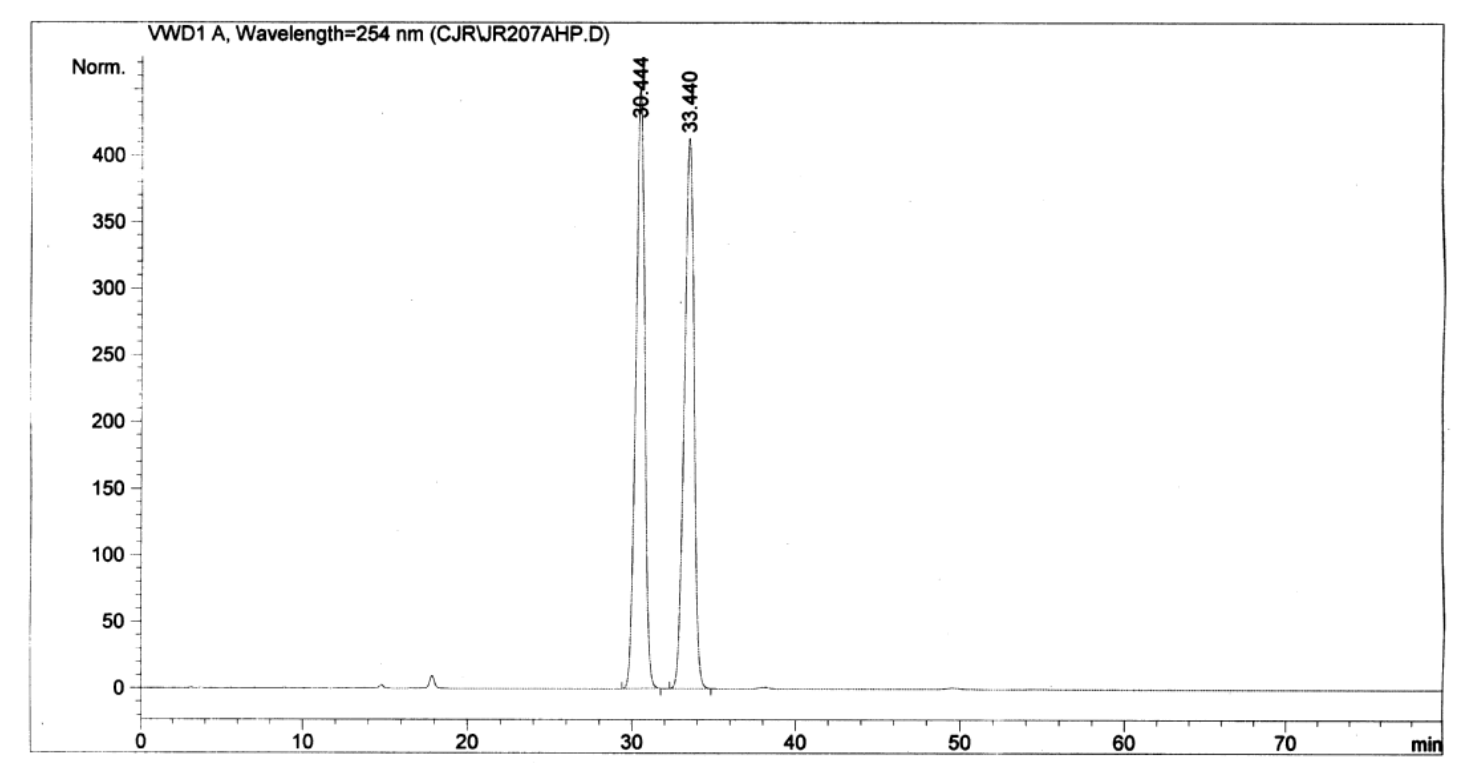<smiles>O=C1CCCCC1C(O)c1cccc([N+](=O)[O-])c1</smiles>

racemic-syn-4c

Signal 1: VWD1 A, Wavelength=254 nm

$$
\begin{aligned}
& \text { Peak RetTime Type Width Area Height Area } \\
& \text { \# [min] [min] mAU *s [mAU ] } \\
& \begin{array}{lllllll}
1 & 30.444 & \text { BB } & 0.5895 & 1.70919 \mathrm{e} 4 & 452.20737 & 50.0341
\end{array}
\end{aligned}
$$

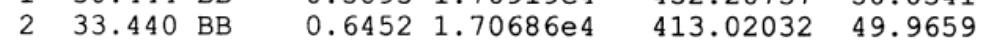

$$
\begin{aligned}
& \text { Totals : } \quad 3.41605 \mathrm{e} 4 \quad 865.22769
\end{aligned}
$$

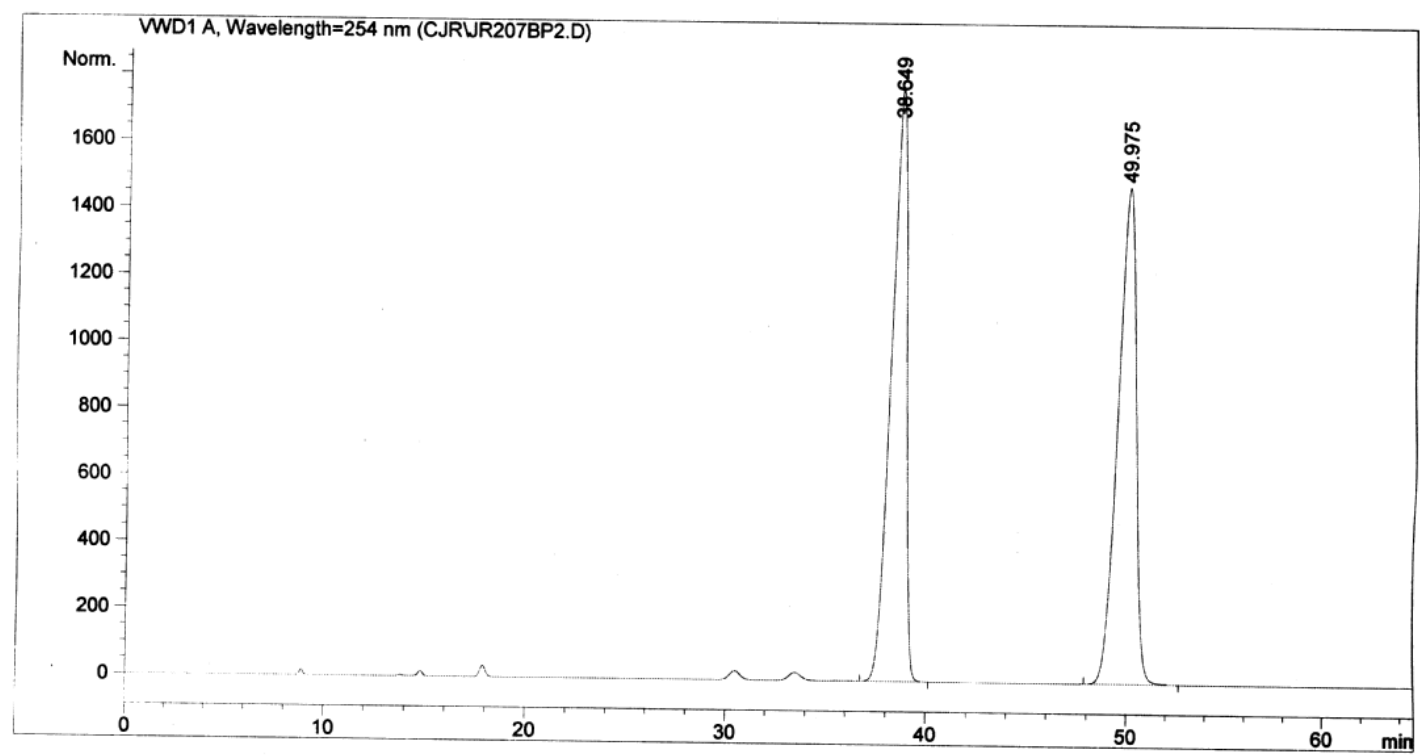<smiles>O=C1CCCCC1C(O)c1cccc([N+](=O)[O-])c1</smiles>

racemic-anti-4c

Signal 1: VWD1 A, Wavelength=254 nm

$$
\begin{aligned}
& \begin{array}{c}
\text { Peak RetTime Type Width Area Height Area } \\
\# \text { [min] }
\end{array}
\end{aligned}
$$

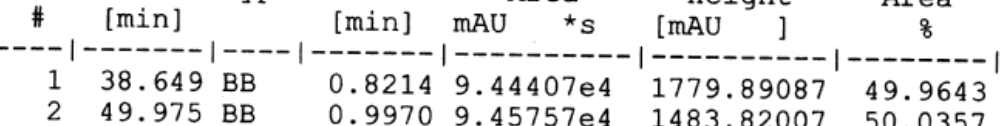

$$
\begin{aligned}
& \text { Totals : } \quad 1.89016 \mathrm{e} 5 \quad 3263.71094
\end{aligned}
$$




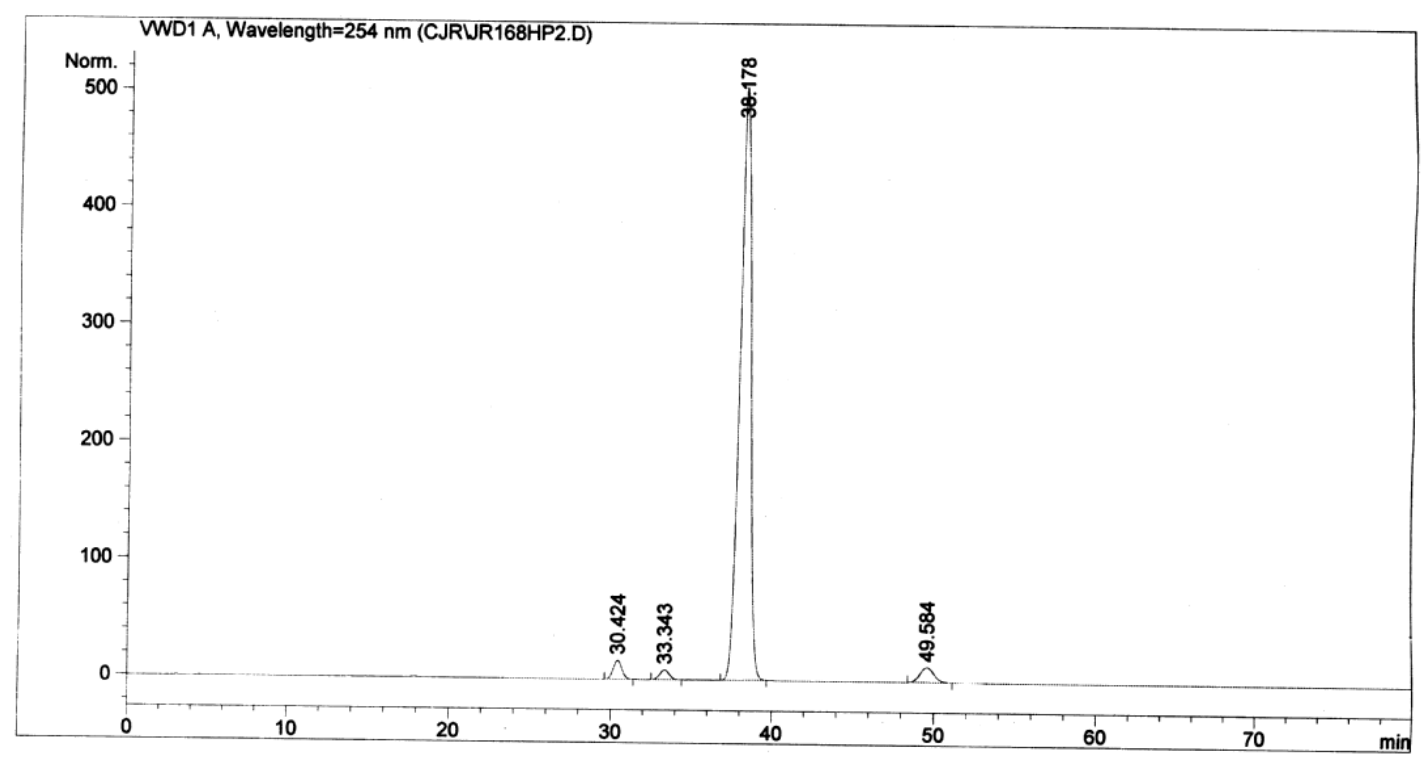<smiles>O=C1CCCC[C@H]1C(O)c1cccc([N+](=O)[O-])c1</smiles>

Signal 1: VWD1 A, Wavelength=254 nm

\begin{tabular}{|c|c|c|c|c|c|c|}
\hline $\begin{array}{c}\text { Peak } \\
\#\end{array}$ & $\begin{array}{l}\text { RetTime } \\
\text { [min] }\end{array}$ & Type & $\begin{array}{l}\text { Width } \\
\text { [min] }\end{array}$ & ${ }_{\mathrm{mAU}}{ }^{\text {Area }}{ }^{*} \mathrm{~s}$ & $\begin{array}{l}\text { Height } \\
{[\mathrm{mAU}]}\end{array}$ & $\begin{array}{c}\text { Area } \\
\frac{8}{8}\end{array}$ \\
\hline $\begin{array}{l}1 \\
2 \\
3 \\
4\end{array}$ & $\begin{array}{l}30.424 \\
33.343 \\
38.178 \\
49.584\end{array}$ & $\begin{array}{l}\text { BB } \\
\text { BB } \\
\text { BB } \\
\text { BB }\end{array}$ & $\begin{array}{l}0.5749 \\
0.6321 \\
0.7376 \\
0.9030\end{array}$ & $\begin{array}{c}602.79755 \\
341.79663 \\
2.38550 \mathrm{e} 4 \\
775.31104\end{array}$ & $\begin{array}{r}16.21706 \\
8.37158 \\
506.19727 \\
12.99155\end{array}$ & $\begin{array}{r}2.3570 \\
1.3365 \\
93.2750 \\
3.0315\end{array}$ \\
\hline Tot & $s:$ & & & $2.55749 \mathrm{e} 4$ & 543.77746 & \\
\hline
\end{tabular}

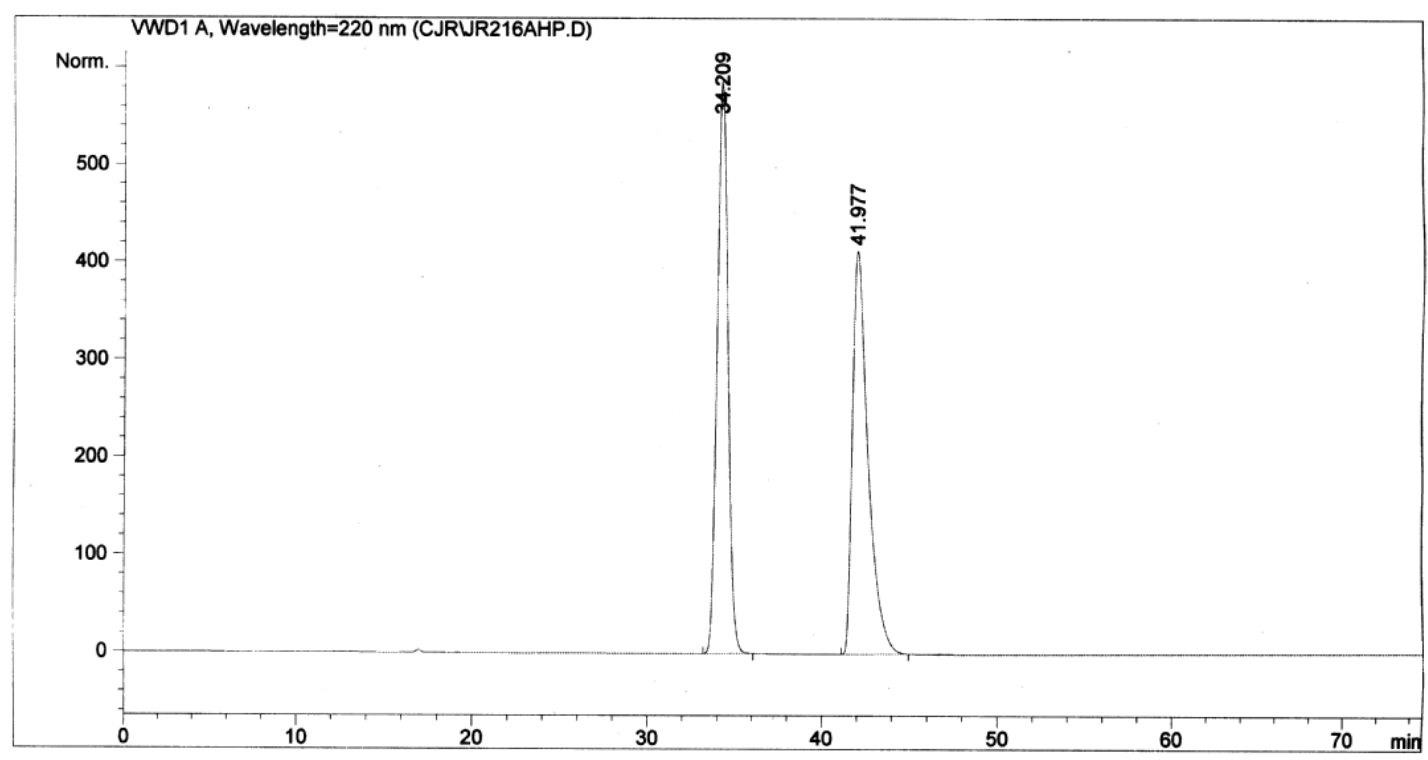<smiles>N#Cc1ccc(C(O)C2CCCCC2=O)cc1</smiles>

racemic-syn-4d

Signal 1: VWD1 A, Wavelength $=220 \mathrm{~nm}$

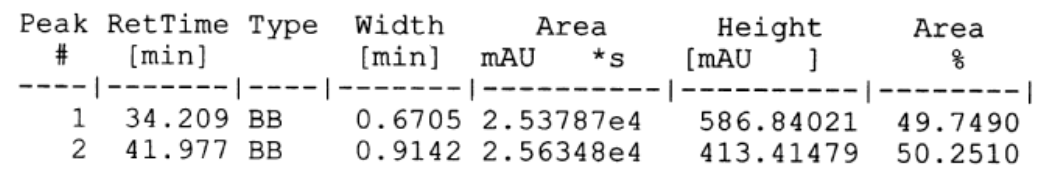

$$
\begin{aligned}
& \text { Totals : } \\
& 5.10135 \mathrm{e} 4 \quad 1000.25500
\end{aligned}
$$



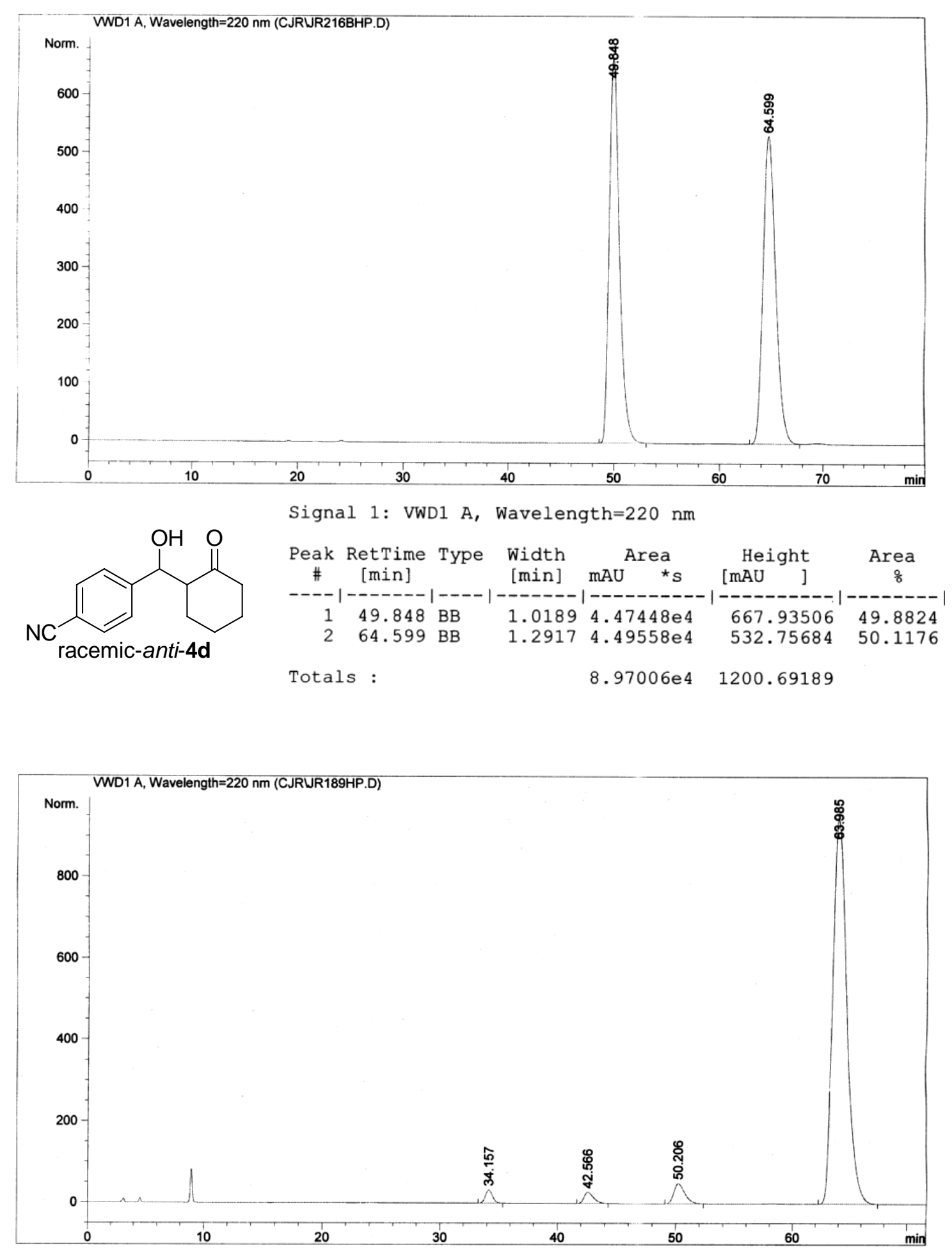

Signal 1: VWD1 A, Wavelength=220 nm

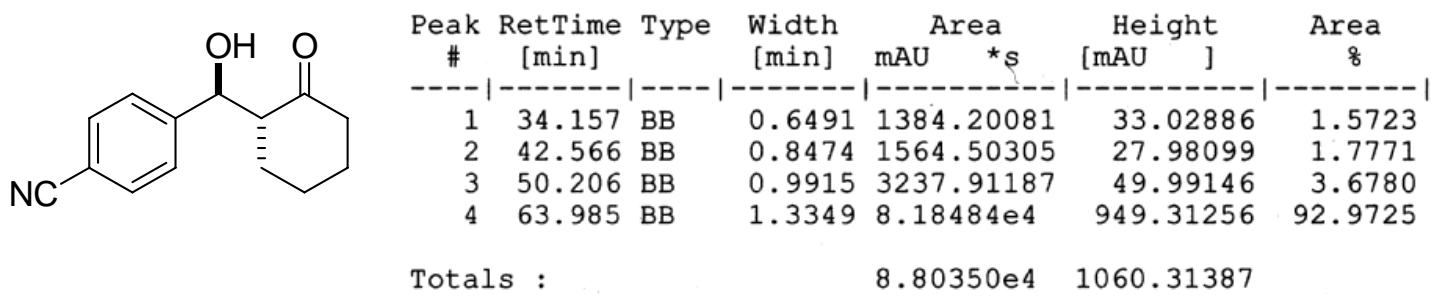




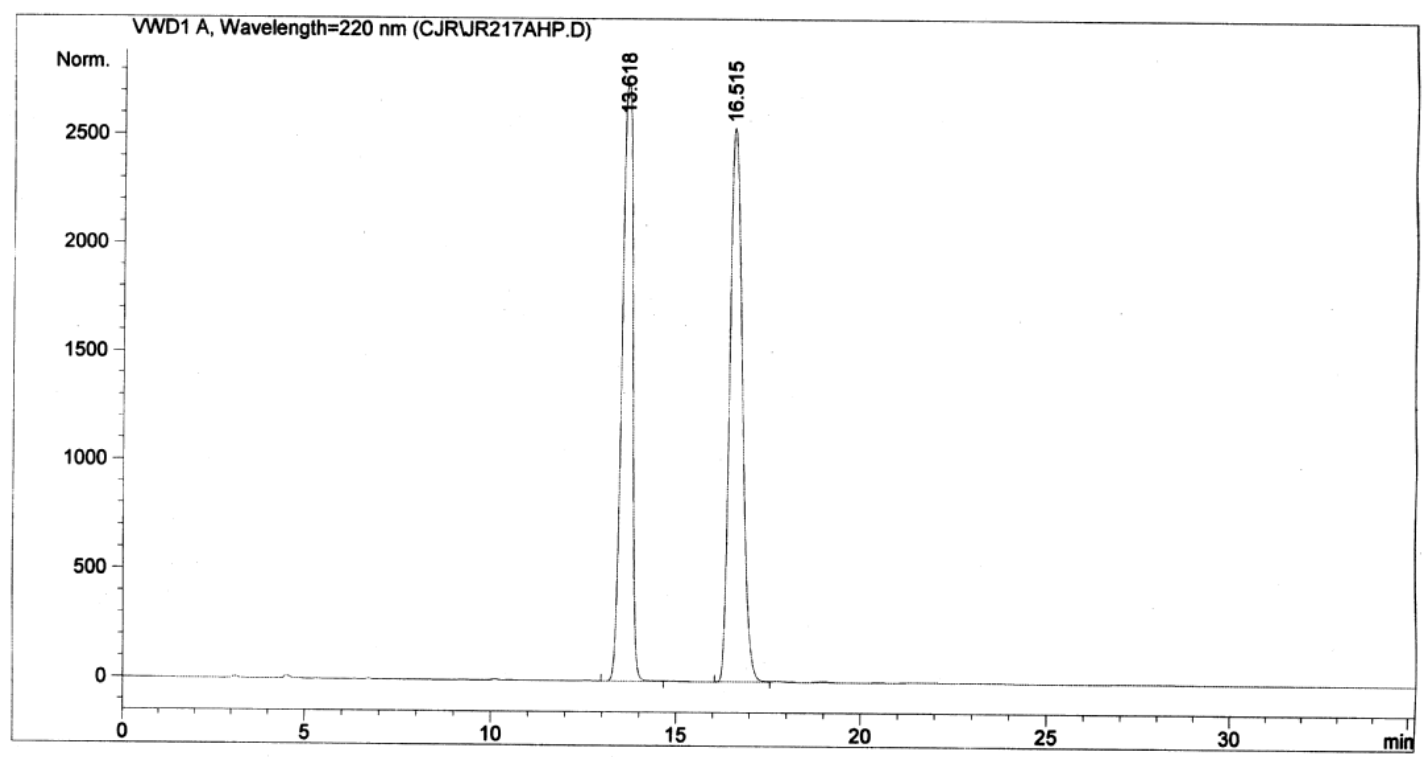<smiles>O=C1CCCCC1C(O)c1ccc(Cl)cc1</smiles>

Signal 1: VWD1 A, Wavelength=220 nm

racemic-syn-4e

$$
\begin{aligned}
& \text { Peak RetTime Type Width Area Height Area } \\
& \text { \# [min] [min] } \mathrm{mAU}{ }^{*} \mathrm{~S} \text { [mAU ] o } \\
& \begin{array}{llllll}
13.618 \mathrm{VV} & 0.3181 & 5.52157 \mathrm{e} 4 & 2756.64063 & 47.6429
\end{array} \\
& \begin{array}{llllll}
2 & 16.515 \mathrm{PV} & 0.3679 & 6.06793 \mathrm{e} 4 & 2549.67578 & 52.3571
\end{array}
\end{aligned}
$$

\begin{tabular}{|c|c|c|c|c|c|c|}
\hline $\begin{array}{c}\text { Peak } \\
\#\end{array}$ & $\begin{array}{l}\text { RetTime } \\
\text { [min] }\end{array}$ & Type & $\begin{array}{l}\text { Width } \\
\text { [min] }\end{array}$ & $\mathrm{mAU}^{\text {Area }}{ }^{*} \mathrm{~s}$ & $\underset{[\mathrm{mAU}]}{\text { Height }}$ & $\begin{array}{c}\text { Area } \\
\frac{8}{8}\end{array}$ \\
\hline $\begin{array}{l}1 \\
2\end{array}$ & $\begin{array}{l}22.621 \\
26.803\end{array}$ & $\begin{array}{l}\text { BB } \\
\text { BB }\end{array}$ & $\begin{array}{l}0.4551 \\
0.5381\end{array}$ & $\begin{array}{l}5.77199 \mathrm{e} 4 \\
5.84280 \mathrm{e} 4\end{array}$ & $\begin{array}{l}1980.27515 \\
1686.79199\end{array}$ & $\begin{array}{l}49.6951 \\
50.3049\end{array}$ \\
\hline Tota & : & & & $1.16148 \mathrm{e} 5$ & 3667.06714 & \\
\hline
\end{tabular}

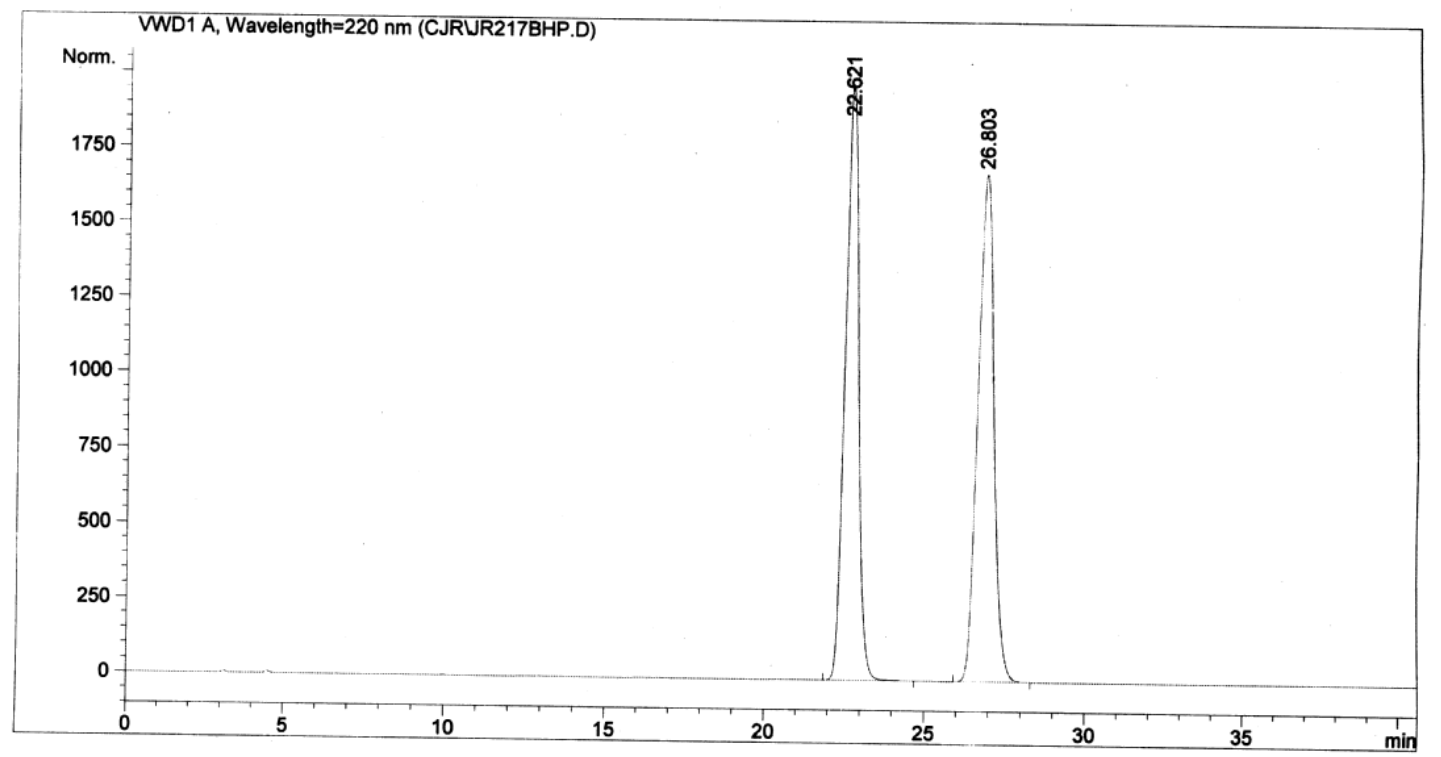<smiles>O=C1CCCCC1C(O)c1ccc(Cl)cc1</smiles>

racemic-anti-4e

Signal 1: VWD1 A, Wavelength=220 nm 


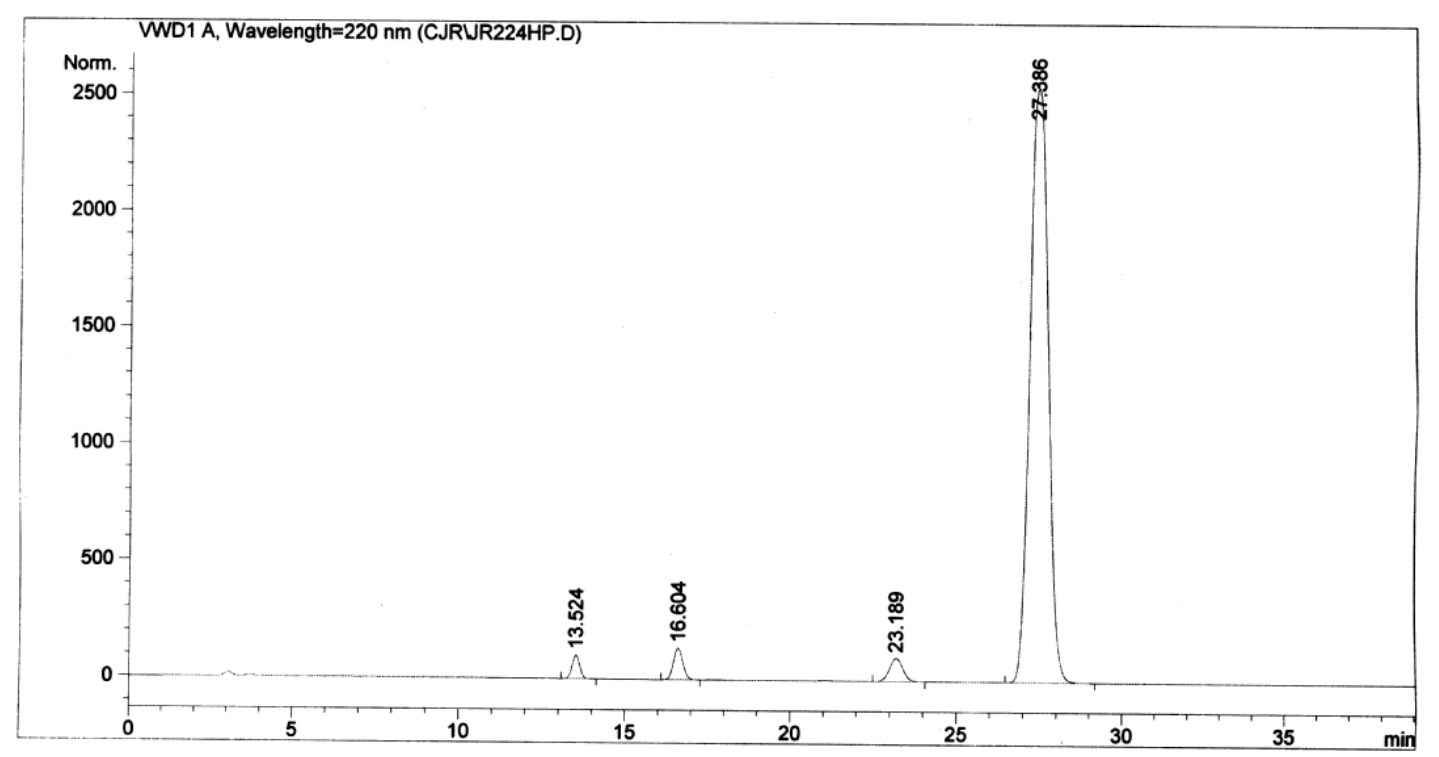

Signal 1: VWD1 A, Wavelength=220 nm<smiles>O=C1CCCC[C@H]1C(O)c1ccc(Cl)cc1</smiles>

\begin{tabular}{|c|c|c|c|c|c|c|}
\hline $\begin{array}{c}\text { Peak } \\
\#\end{array}$ & $\begin{array}{l}\text { RetTime } \\
\text { [min] }\end{array}$ & Type & $\begin{array}{l}\text { Width } \\
\text { [min] }\end{array}$ & ${ }_{\mathrm{mAU}}{ }^{\text {Area }}{ }^{\star} \mathrm{s}$ & $\begin{array}{c}\text { Height } \\
{[\mathrm{mAU}]}\end{array}$ & $\begin{array}{c}\text { Area } \\
\frac{8}{8}\end{array}$ \\
\hline $\begin{array}{l}1 \\
2 \\
3 \\
4\end{array}$ & $\begin{array}{l}13.524 \\
16.604 \\
23.189 \\
27.386\end{array}$ & $\begin{array}{l}\text { VB } \\
\text { PB } \\
\text { BB } \\
\text { BB }\end{array}$ & $\begin{array}{l}0.2510 \\
0.3126 \\
0.4331 \\
0.6066\end{array}$ & $\begin{array}{l}1629.37964 \\
2717.63208 \\
2802.41138 \\
9.78571 \mathrm{e} 4\end{array}$ & $\begin{array}{r}101.70869 \\
135.48967 \\
100.94766 \\
2549.02539\end{array}$ & $\begin{array}{r}1.5517 \\
2.5881 \\
2.6688 \\
93.1915\end{array}$ \\
\hline Tota & s : & & & 1.05007 e5 & 2887.17141 & \\
\hline
\end{tabular}

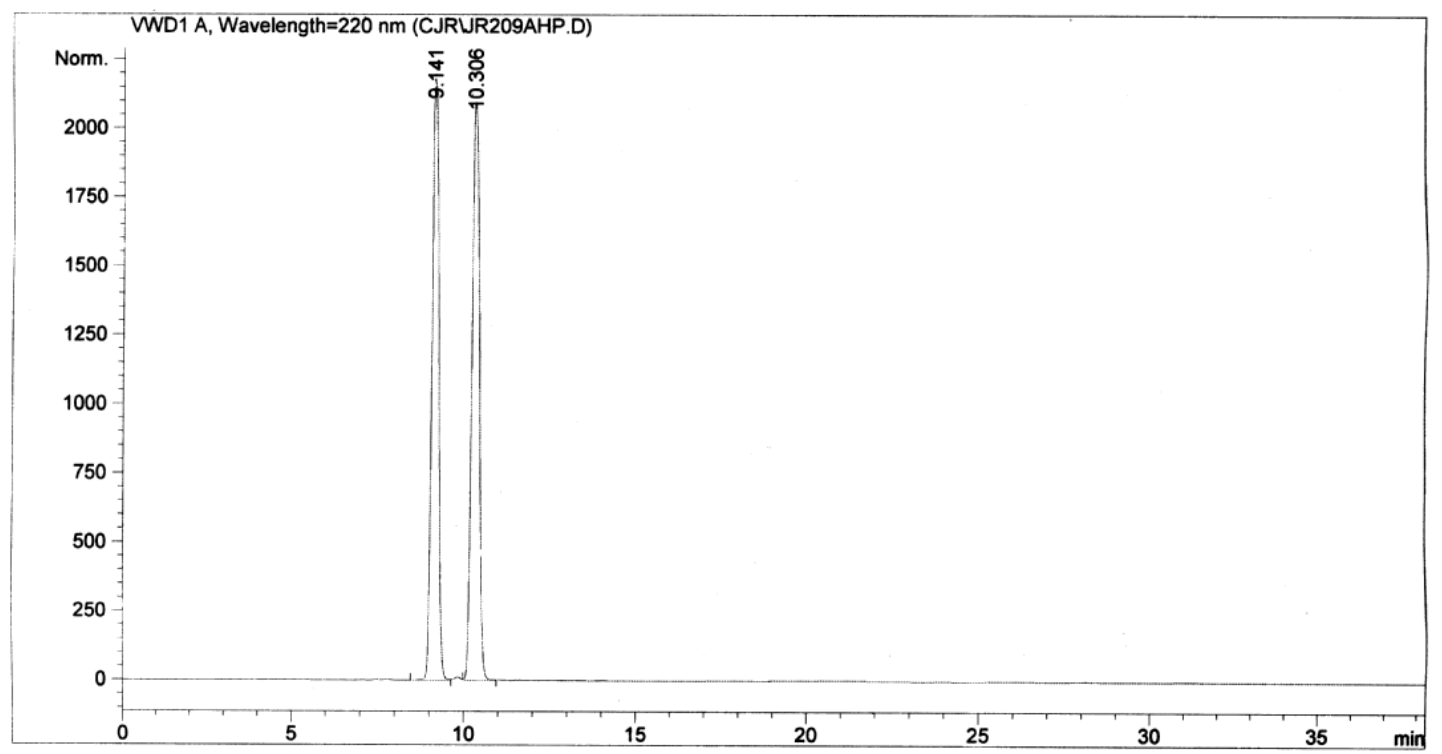<smiles>O=C1CCCCC1C(O)c1ccccc1Cl</smiles>

racemic-syn-4f

Signal 1: VWD1 A, Wavelength=220 nm

\begin{tabular}{|c|c|c|c|c|c|c|}
\hline $\begin{array}{c}\text { Peak } \\
\#\end{array}$ & RetTime & Type & Width & Area & Height & $\begin{array}{c}\text { Area } \\
\frac{8}{\delta}\end{array}$ \\
\hline & & & & |--------- & --------- & \\
\hline $\begin{array}{l}1 \\
2\end{array}$ & $\begin{array}{r}9.141 \\
10.306\end{array}$ & $\begin{array}{l}\text { BV } \\
\text { VB }\end{array}$ & $\begin{array}{l}0.2215 \\
0.2416\end{array}$ & $\begin{array}{l}3.00159 \mathrm{e} 4 \\
3.14119 \mathrm{e} 4\end{array}$ & $\begin{array}{l}2183.82300 \\
2098.57910\end{array}$ & $\begin{array}{l}48.8637 \\
51.1363\end{array}$ \\
\hline Total & Is : & & & $6.14278 \mathrm{e} 4$ & 4282.40210 & \\
\hline
\end{tabular}




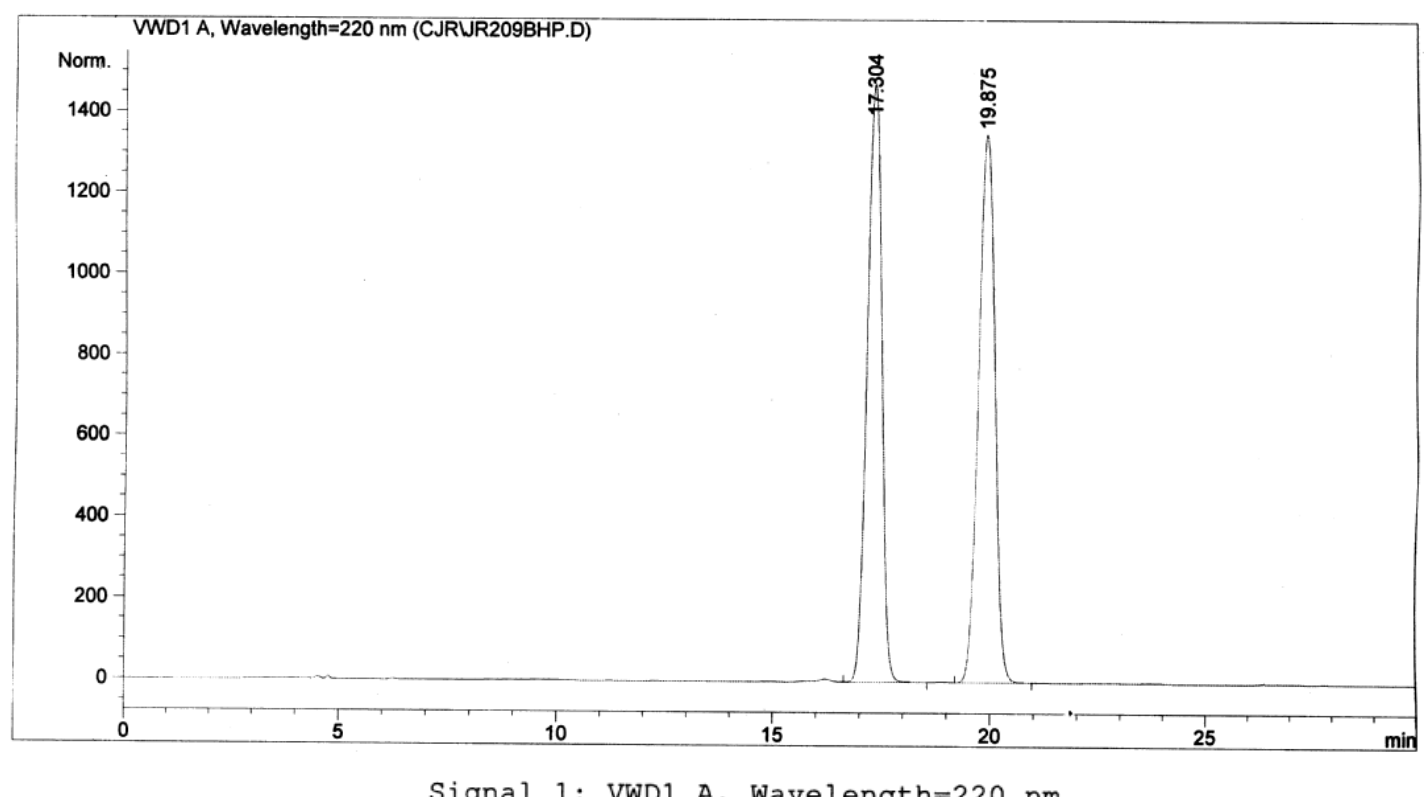<smiles>O=C1CCCCC1C(O)c1ccccc1Cl</smiles>

racemic-anti-4f

elength $=220 \mathrm{n}$

$$
\begin{aligned}
& \text { Peak RetTime Type Width Area Height Area } \\
& \text { \#[min] [min] mAU *s [mAU ] } 8 \\
& \begin{array}{llllllll}
1 & 17.304 & \text { VV } & 0.3740 & 3.43993 \mathrm{e} 4 & 1474.77673 & 49.3774
\end{array} \\
& \begin{array}{lllllll}
2 & 19.875 & \text { VB } & 0.4153 & 3.52668 \mathrm{e} 4 & 1350.62207 & 50.6226
\end{array}
\end{aligned}
$$

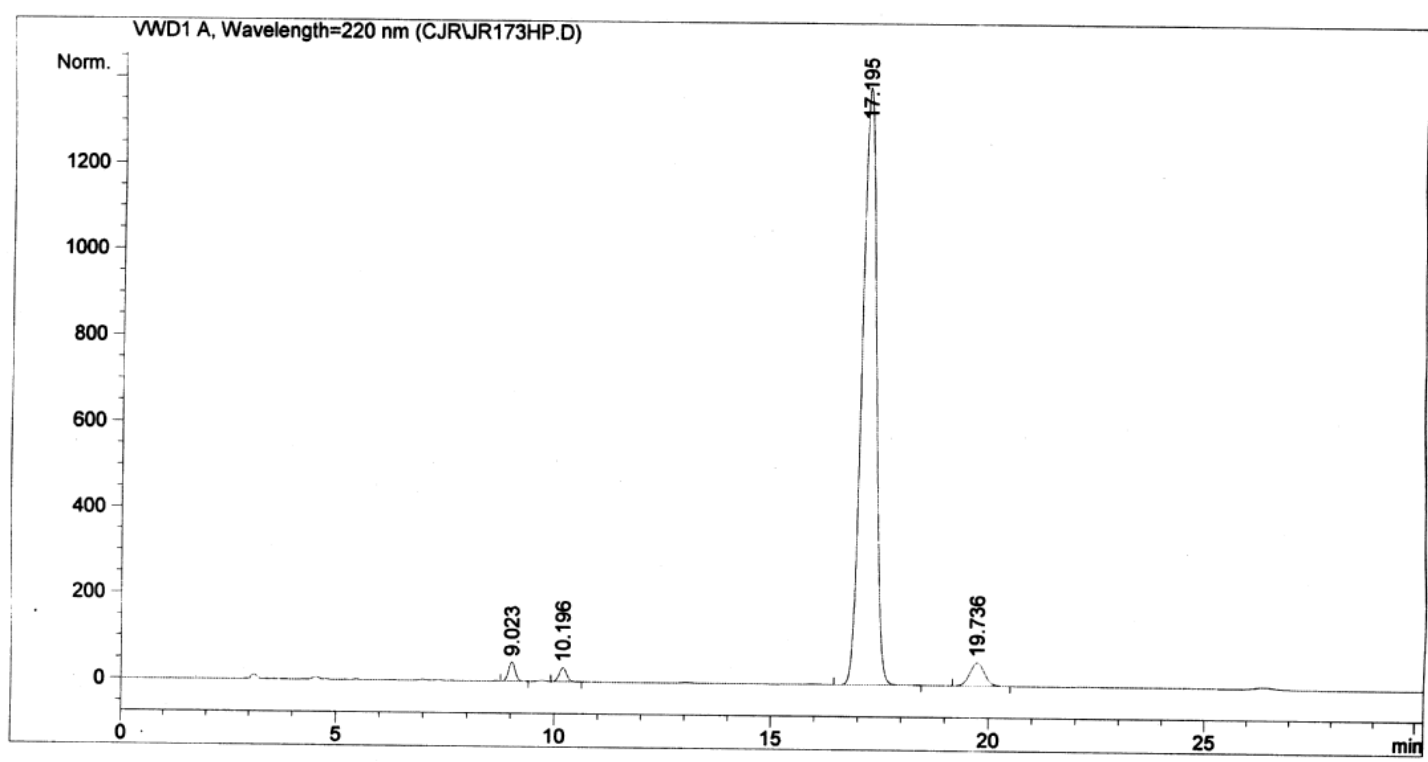

Signal 1: VWD1 A, Wavelength=220 nm<smiles>O=C1CCCC[C@H]1C(O)c1ccccc1Cl</smiles>

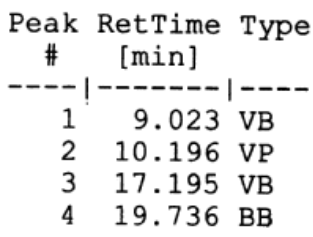

Totals :

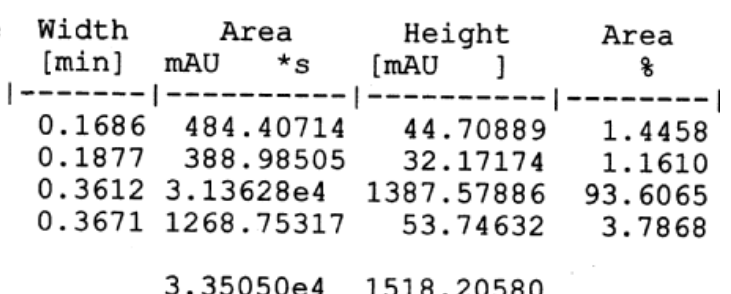

$3.35050 \mathrm{e} 4 \quad 1518.20580$ 

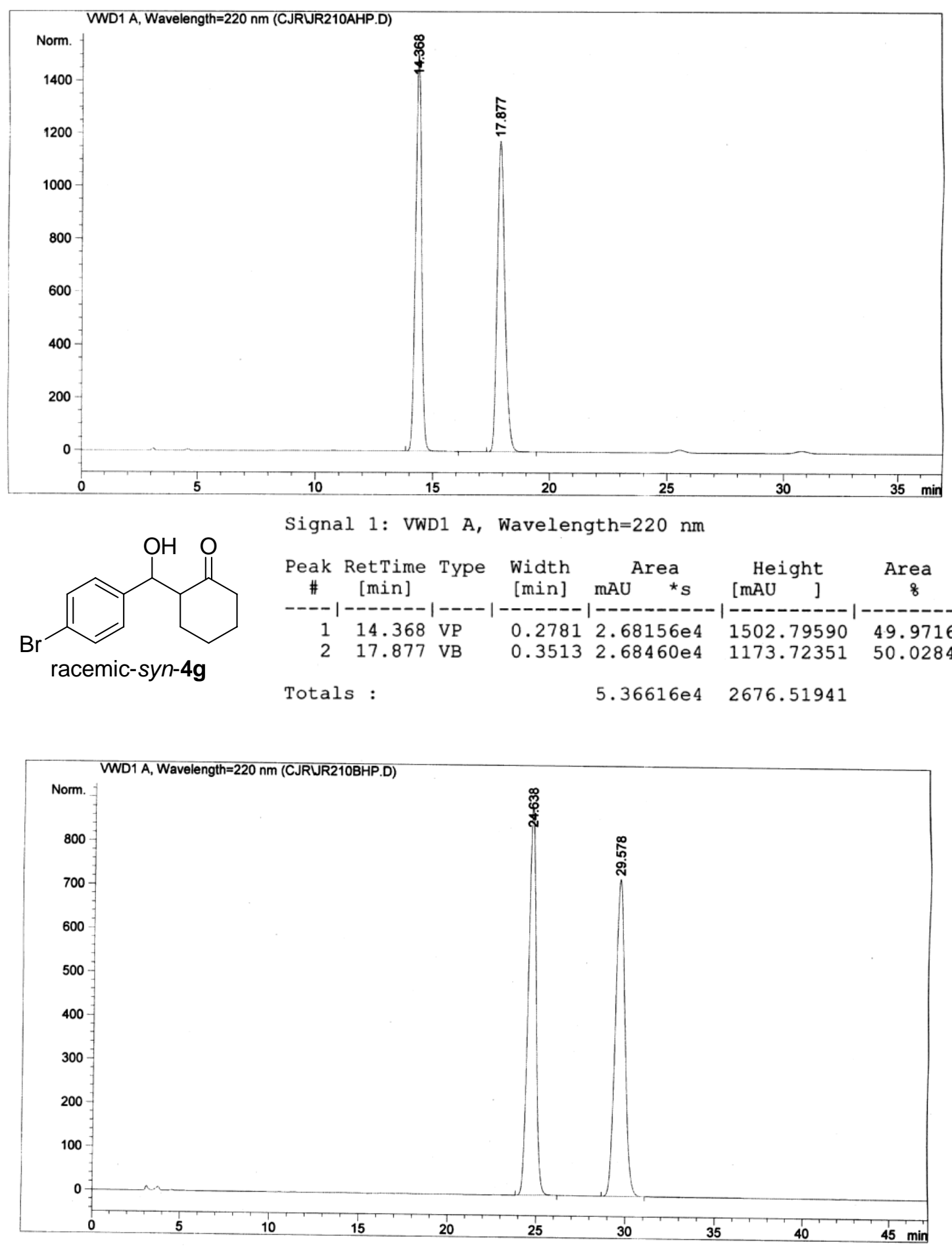<smiles>O=C1CCCCC1C(O)c1ccc(Br)cc1</smiles>

racemic-anti-4g

Signal 1: VWD1 $A$, Wavelength $=220 \mathrm{~nm}$<smiles>O=C1CCCC(C(O)c2ccc(Br)cc2)C1C(=O)OCc1ccccc1</smiles>

Totals

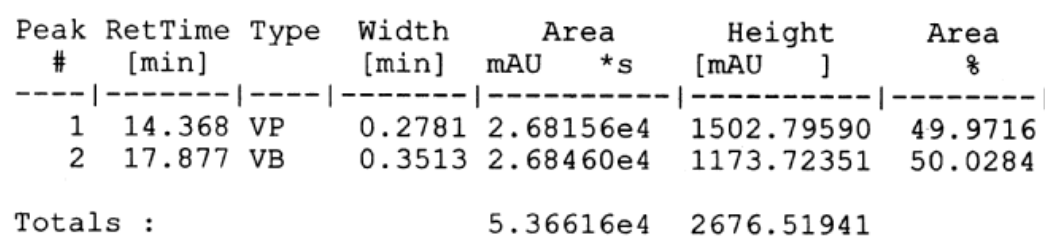

Signal 1: VWD1 A, Wavelength=220 nm

\begin{tabular}{|c|c|c|c|c|c|c|}
\hline $\begin{array}{c}\text { Peak } \\
\quad \#\end{array}$ & $\begin{array}{l}\text { RetTime } \\
\text { [min] }\end{array}$ & Type & $\begin{array}{l}\text { Width } \\
\text { [min] }\end{array}$ & $\mathrm{mAU}^{\text {Area }}{ }^{\star} \mathrm{S}$ & $\begin{array}{l}\text { Height } \\
{[\mathrm{mAU}]}\end{array}$ & $\underset{8}{\text { Area }}$ \\
\hline $\begin{array}{l}1 \\
2\end{array}$ & $\begin{array}{l}24.638 \\
29.578\end{array}$ & $\begin{array}{l}\text { BB } \\
\text { BB }\end{array}$ & $\begin{array}{l}0.4730 \\
0.5790\end{array}$ & $\begin{array}{l}2.70247 \mathrm{e} 4 \\
2.70931 \mathrm{e} 4\end{array}$ & $\begin{array}{l}888.05933 \\
724.42871\end{array}$ & $\begin{array}{l}49.9368 \\
50.0632\end{array}$ \\
\hline Tota & : & & & $5.41178 \mathrm{e} 4$ & 1612.48804 & \\
\hline
\end{tabular}




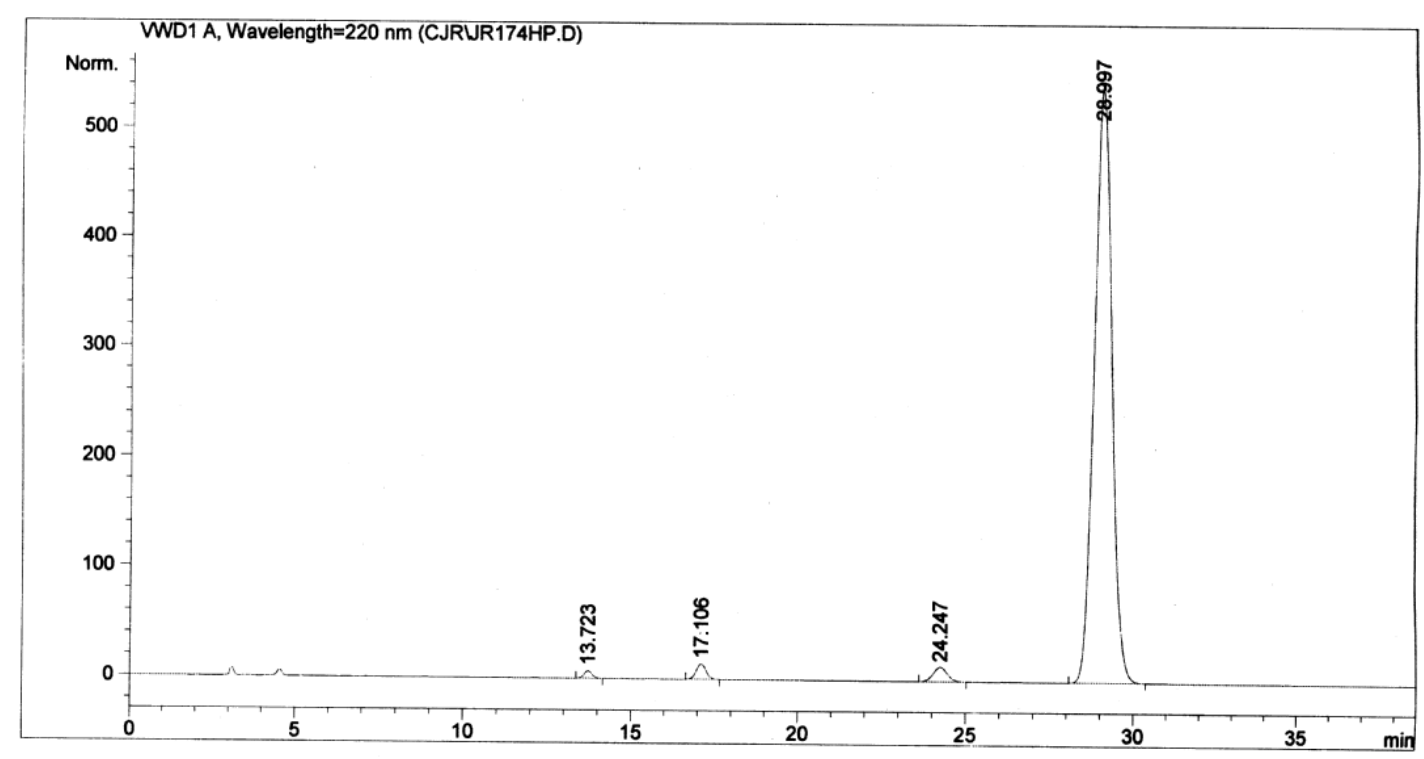

Signal 1: VWD1 A, Wavelength=220 nm
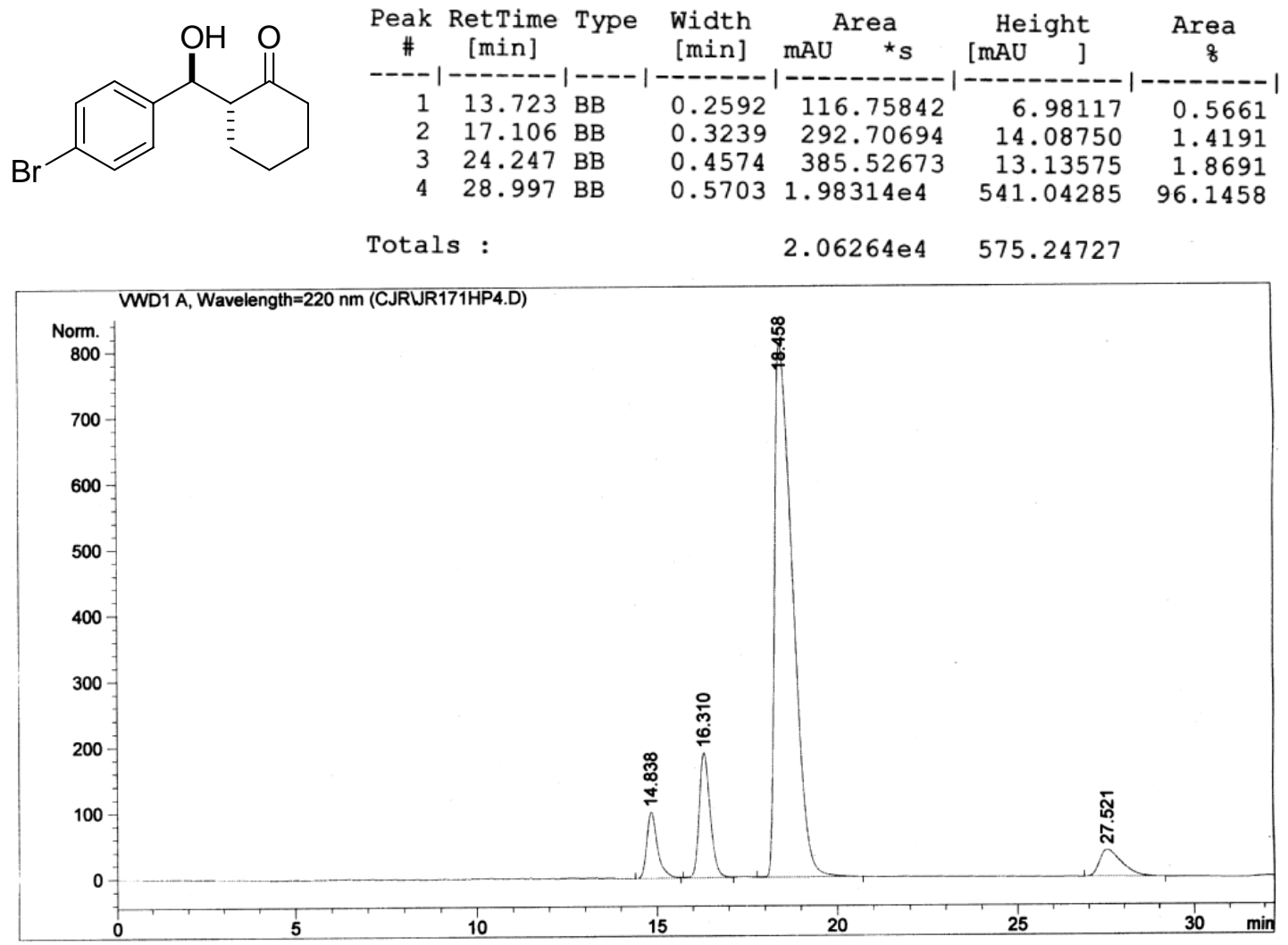

Signal 1: VWD1 A, Wavelength=220 nm<smiles>O=C1CCCC[C@H]1C(O)c1ccccc1</smiles>

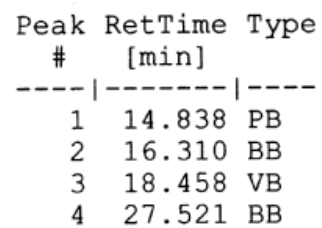

Totals :

\begin{tabular}{|c|c|c|c|}
\hline \multirow{2}{*}{$\begin{array}{c}\text { Width } \\
\text { [min] }\end{array}$} & Area & Height & \multirow{2}{*}{$\begin{array}{c}\text { Area } \\
\frac{8}{8}\end{array}$} \\
\hline & $\mathrm{mAU} \quad$ *s & {$[\mathrm{mAU}]$} & \\
\hline & .68579 & 101.02 & 410 \\
\hline & 406 & & \\
\hline 0.5482 & $\begin{array}{l}2.79970 \mathrm{e} 4 \\
1798 \quad 76489\end{array}$ & $\begin{array}{r}811.31171 \\
40.84850\end{array}$ & $\begin{array}{r}77.8464 \\
5.0015\end{array}$ \\
\hline
\end{tabular}

$3.59644 \mathrm{e} 4 \quad 1143.23704$ 


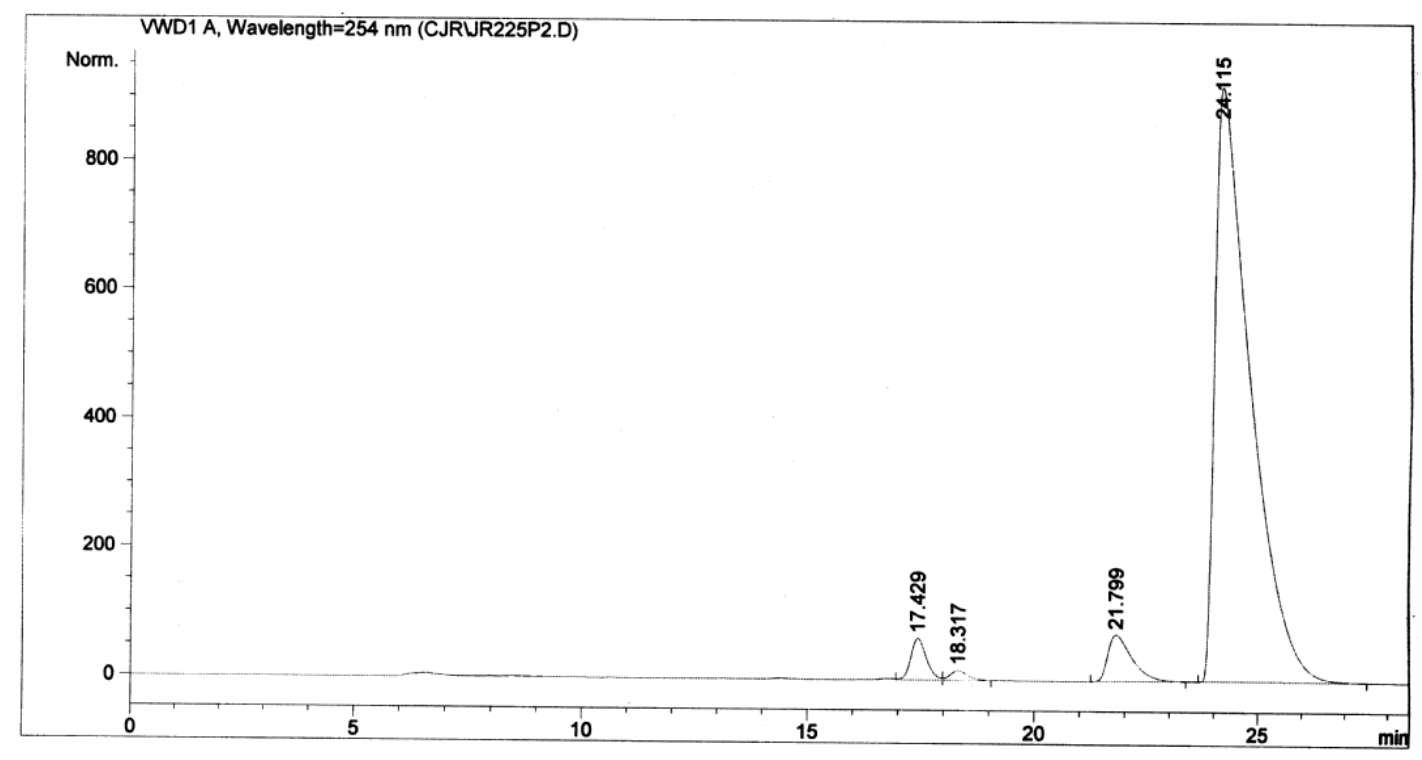

Signal 1: VWD1 A, Wavelength=254 nm<smiles>O=C1CCCC[C@H]1C(O)c1cccc2ccccc12</smiles>

\begin{tabular}{|c|c|c|c|c|c|c|}
\hline $\begin{array}{c}\text { Peak } \\
\#\end{array}$ & $\begin{array}{l}\text { RetTime } \\
\text { [min] }\end{array}$ & Type & $\begin{array}{l}\text { Width } \\
\text { [min] }\end{array}$ & 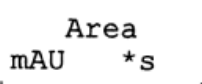 & $\begin{array}{c}\text { Height } \\
{[\mathrm{mAU}]}\end{array}$ & $\begin{array}{c}\text { Area } \\
\frac{8}{8}\end{array}$ \\
\hline $\begin{array}{l}1 \\
2 \\
3 \\
4\end{array}$ & $\begin{array}{l}17.429 \\
18.317 \\
21.799 \\
24.115\end{array}$ & $\begin{array}{l}\text { VV } \\
\text { VB } \\
\text { PB } \\
\text { BB }\end{array}$ & $\begin{array}{l}0.3667 \\
0.3973 \\
0.5647 \\
0.7851\end{array}$ & $\begin{array}{r}1534.24426 \\
394.32300 \\
2701.97119 \\
5.17260 \mathrm{e} 4\end{array}$ & $\begin{array}{r}64.07140 \\
15.12760 \\
71.96593 \\
922.58258\end{array}$ & $\begin{array}{r}2.7224 \\
0.6997 \\
4.7944 \\
91.7835\end{array}$ \\
\hline
\end{tabular}

Totals :

$5.63565 \mathrm{e} 4 \quad 1073.74751$

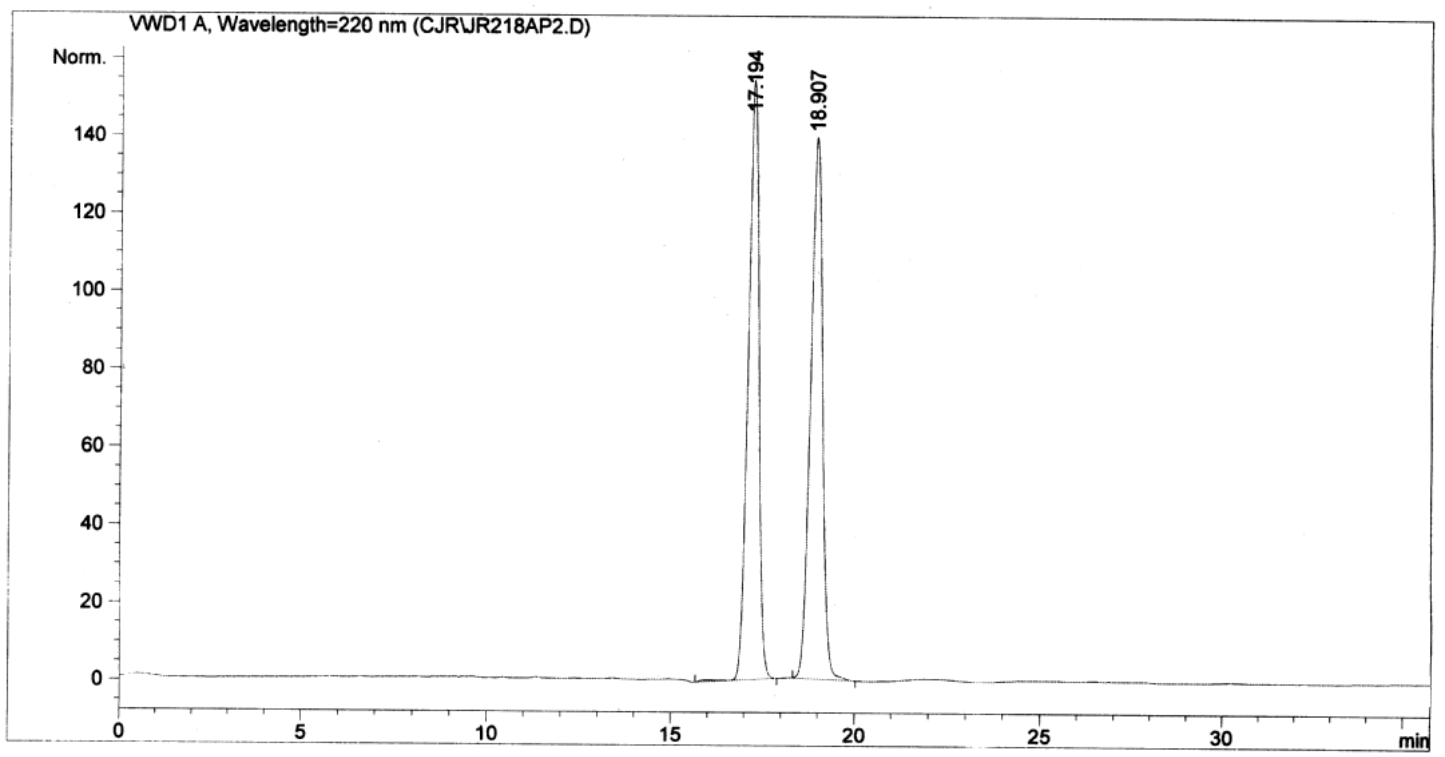<smiles>O=C1CCCCC1C(O)c1ccco1</smiles>

racemic-syn-4j

Signal 1: VWD1 A, Wavelength=220 nm

$$
\begin{aligned}
& \text { Peak RetTime Type Width Area Height Area } \\
& \text { \# [min] [min] mAU *s [mAU ] }{ }^{*} \\
& \begin{array}{llllllll}
1 & 17.194 & \text { BP } & 0.3146 & 3118.77197 & 154.19124 & 49.7612
\end{array} \\
& \begin{array}{lllllll}
2 & 18.907 & \text { BP } & 0.3494 & 3148.70483 & 139.41383 & 50.2388
\end{array} \\
& \text { Totals : } \quad 6267.47681293 .60507
\end{aligned}
$$




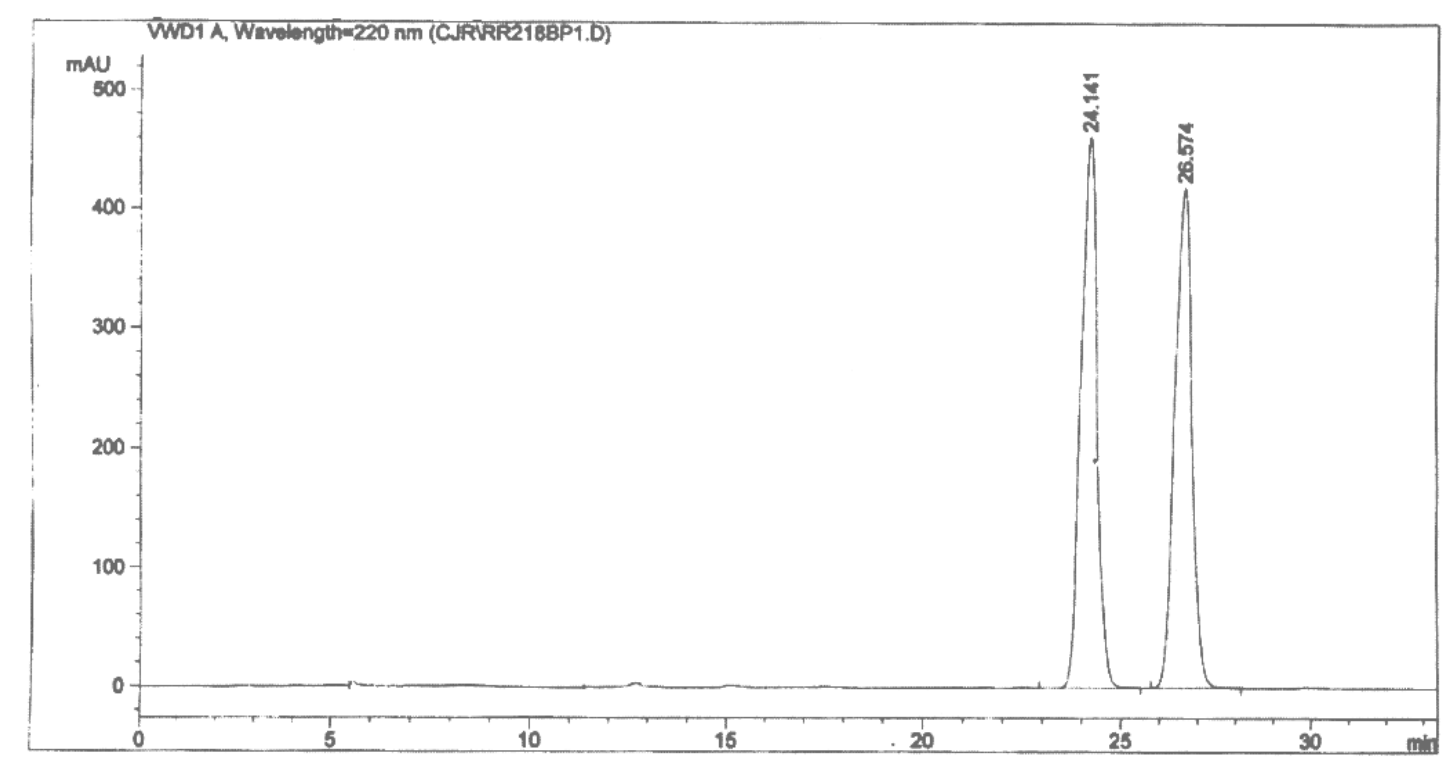<smiles>O=C1CCCCC1C(O)c1ccco1</smiles>

racemic-anti-4j

Signal 1: VhD1 A, Wavelength $=220 \mathrm{~nm}$

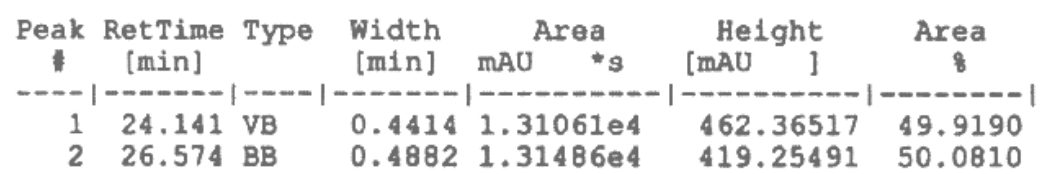

Totals :

$2.62547 e 4 \quad 881.62009$

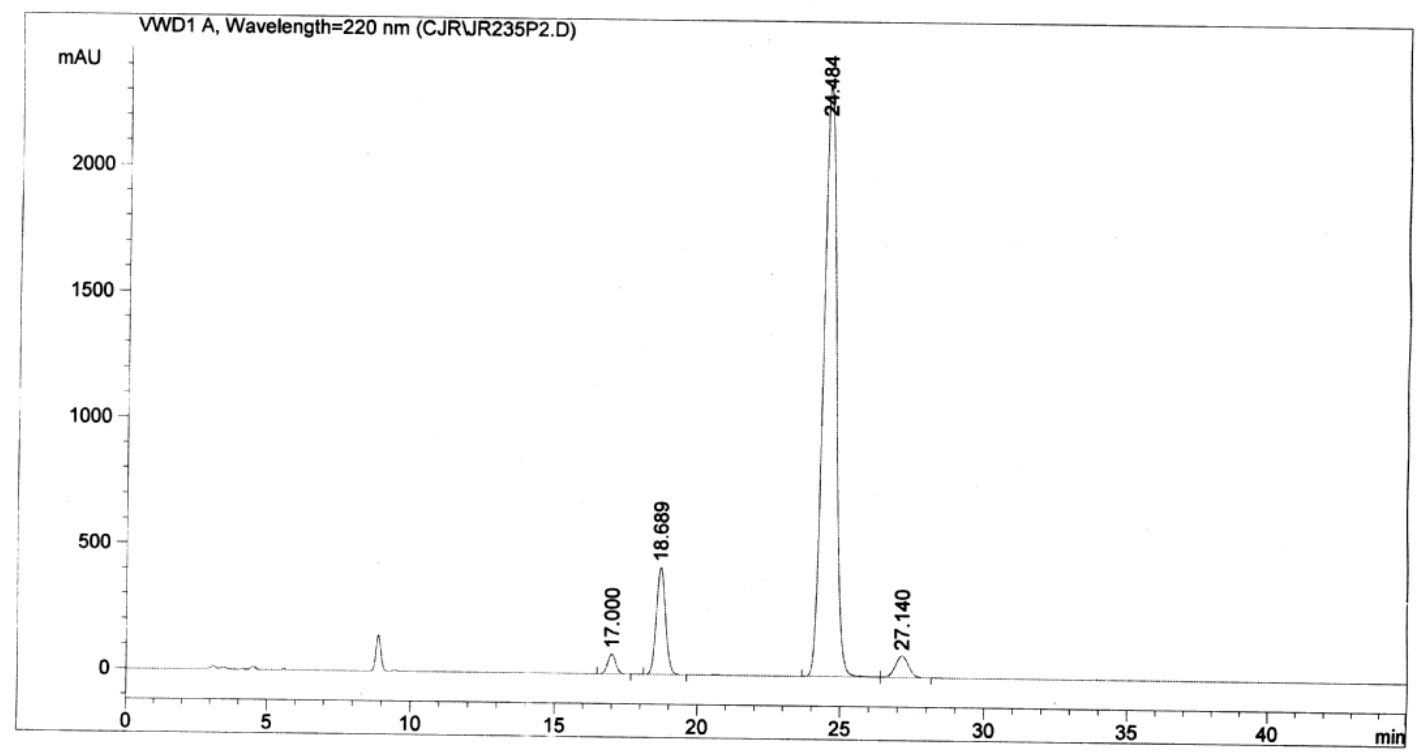

Signal 1: VWD1 A, Wavelength=220 nm<smiles>O=C1CCCC[C@H]1[C@H](O)c1ccco1</smiles>

\begin{tabular}{|c|c|c|c|c|c|c|}
\hline \multirow{2}{*}{$\begin{array}{c}\text { Peak } \\
\#\end{array}$} & \multirow{2}{*}{$\begin{array}{c}\text { RetTime } \\
\text { [min] }\end{array}$} & \multirow{2}{*}{ Type } & \multirow{2}{*}{$\begin{array}{l}\text { Width } \\
\text { [min] }\end{array}$} & Area & Height & \multirow{2}{*}{$\begin{array}{c}\text { Area } \\
\%\end{array}$} \\
\hline & & & & $\mathrm{mAU} \quad$ * $\mathrm{s}$ & {$[\mathrm{mAU} \quad]$} & \\
\hline & & & & & & \\
\hline$\frac{1}{2}$ & 17.000 & $\begin{array}{l}\text { BB } \\
\text { BP }\end{array}$ & 0.3084 & 1559.28235 & 78.66586 & 1.782 \\
\hline 2 & 18.689 & BB & 0.3463 & 9489.65527 & 425.24667 & 10.8506 \\
\hline 3 & 24.484 & BV & 0.4944 & 7.36808 e 4 & 2346.76343 & 84.2474 \\
\hline 4 & 27.140 & VB & 0.4909 & 2727.90210 & 86.00996 & 3.1191 \\
\hline
\end{tabular}




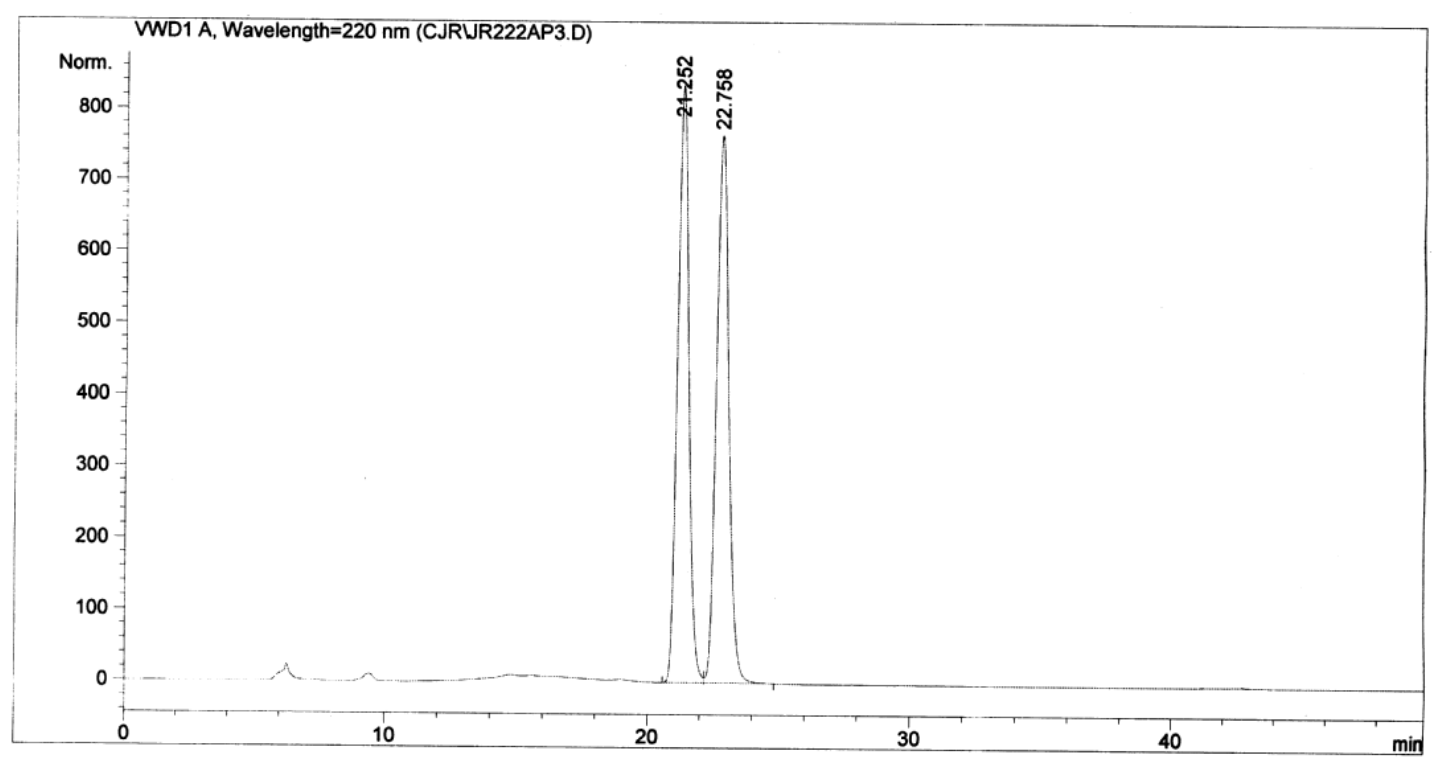<smiles>Cc1ccc(C(O)C2CCCCC2=O)cc1</smiles>

racemic-syn-4k

Signal 1: VWD1 A, Wavelength=220 nm
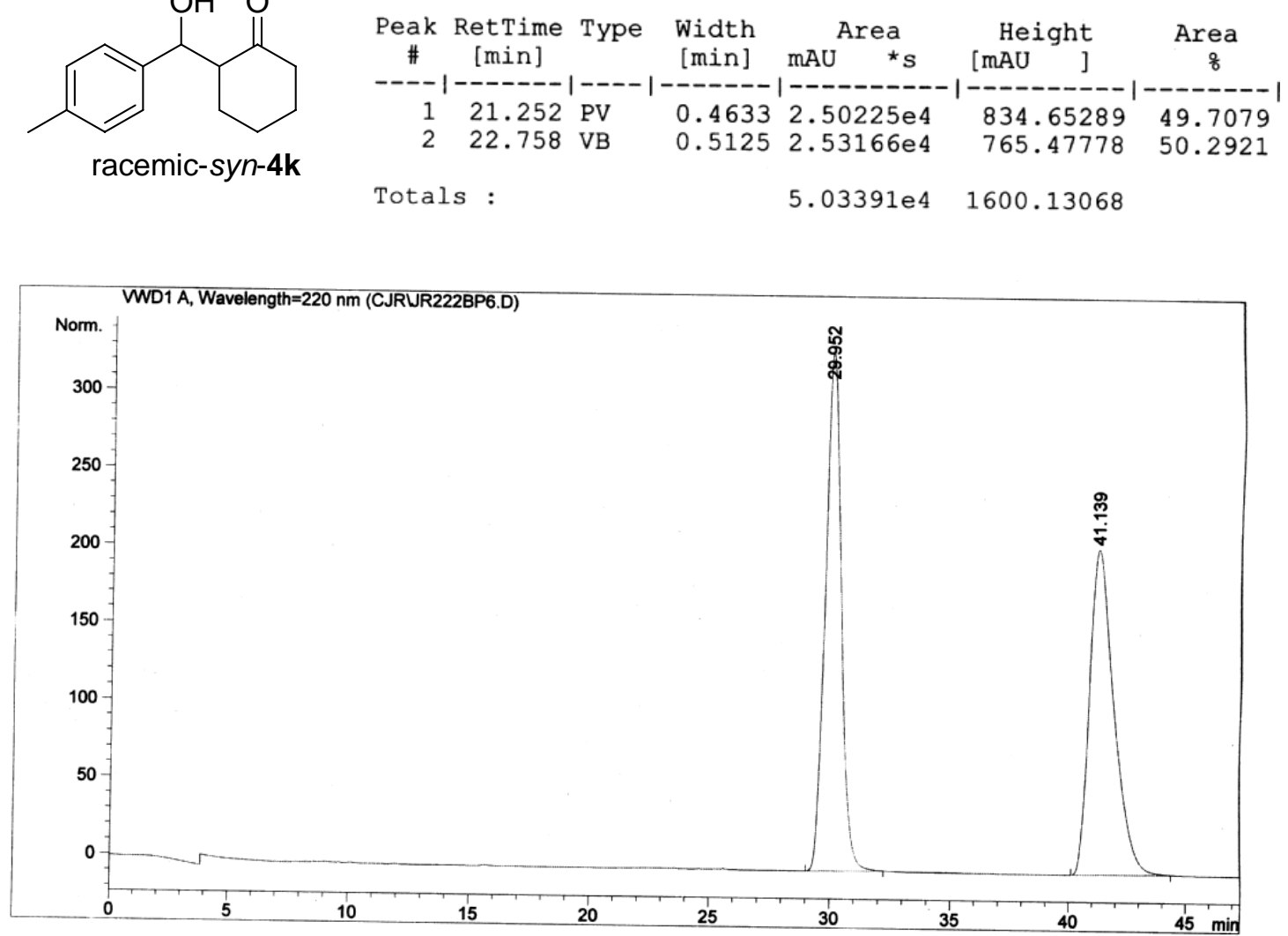<smiles>Cc1ccc(C(O)C2CCCCC2=O)cc1</smiles>

racemic-anti-4k

Signal 1: VWD1 A, Wavelength=220 nm

$$
\begin{aligned}
& \underset{\# \text { Peak RetTime Type Width Area Height Area }}{\text { [min] }}
\end{aligned}
$$

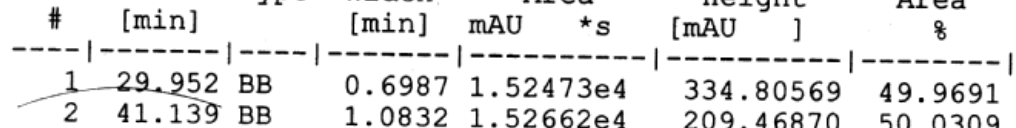

$$
\begin{aligned}
& \text { Totals : } \quad 3.05135 \mathrm{e} 4 \quad 544.27440
\end{aligned}
$$




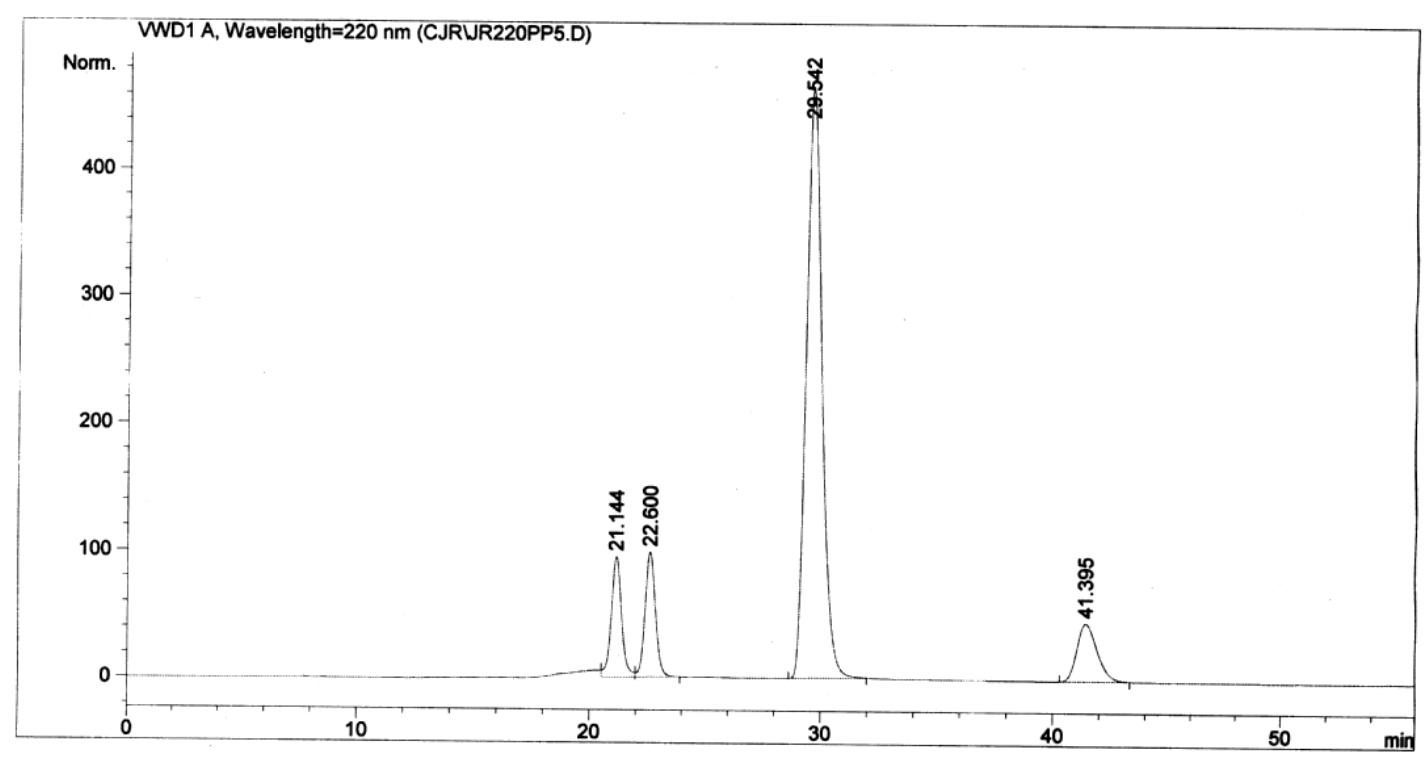

Signal 1: VWD1 $A$, Wavelength $=220 \mathrm{~nm}$<smiles>Cc1ccc(C(O)[C@H]2CCCCC2=O)cc1</smiles>

\begin{tabular}{|c|c|c|c|c|c|c|}
\hline $\begin{array}{c}\text { Peak } \\
\#\end{array}$ & $\begin{array}{l}\text { RetTime } \\
\text { [min] }\end{array}$ & Type & $\begin{array}{l}\text { Width } \\
\text { [min] }\end{array}$ & ${ }_{\mathrm{mAU}}{ }^{\text {Area }}{ }^{\star} \mathrm{s}$ & $\begin{array}{c}\text { Height } \\
{[\mathrm{mAU}]}\end{array}$ & $\begin{array}{c}\text { Area } \\
8\end{array}$ \\
\hline $\begin{array}{l}1 \\
2 \\
3 \\
4\end{array}$ & $\begin{array}{l}21.144 \\
22.600 \\
29.542 \\
41.395\end{array}$ & $\begin{array}{l}\text { BV } \\
\text { VB } \\
\text { BB } \\
\text { BB }\end{array}$ & $\begin{array}{l}0.4780 \\
0.4860 \\
0.7084 \\
0.9388\end{array}$ & $\begin{array}{l}2983.95898 \\
3111.90454 \\
2.18687 \mathrm{e} 4 \\
2791.01514\end{array}$ & $\begin{array}{r}95.13719 \\
98.63985 \\
465.33069 \\
45.66296\end{array}$ & $\begin{array}{r}9.7022 \\
10.1182 \\
71.1048 \\
9.0748\end{array}$ \\
\hline Tot & 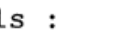 & & & $3.07555 \mathrm{e} 4$ & 704.77068 & \\
\hline
\end{tabular}

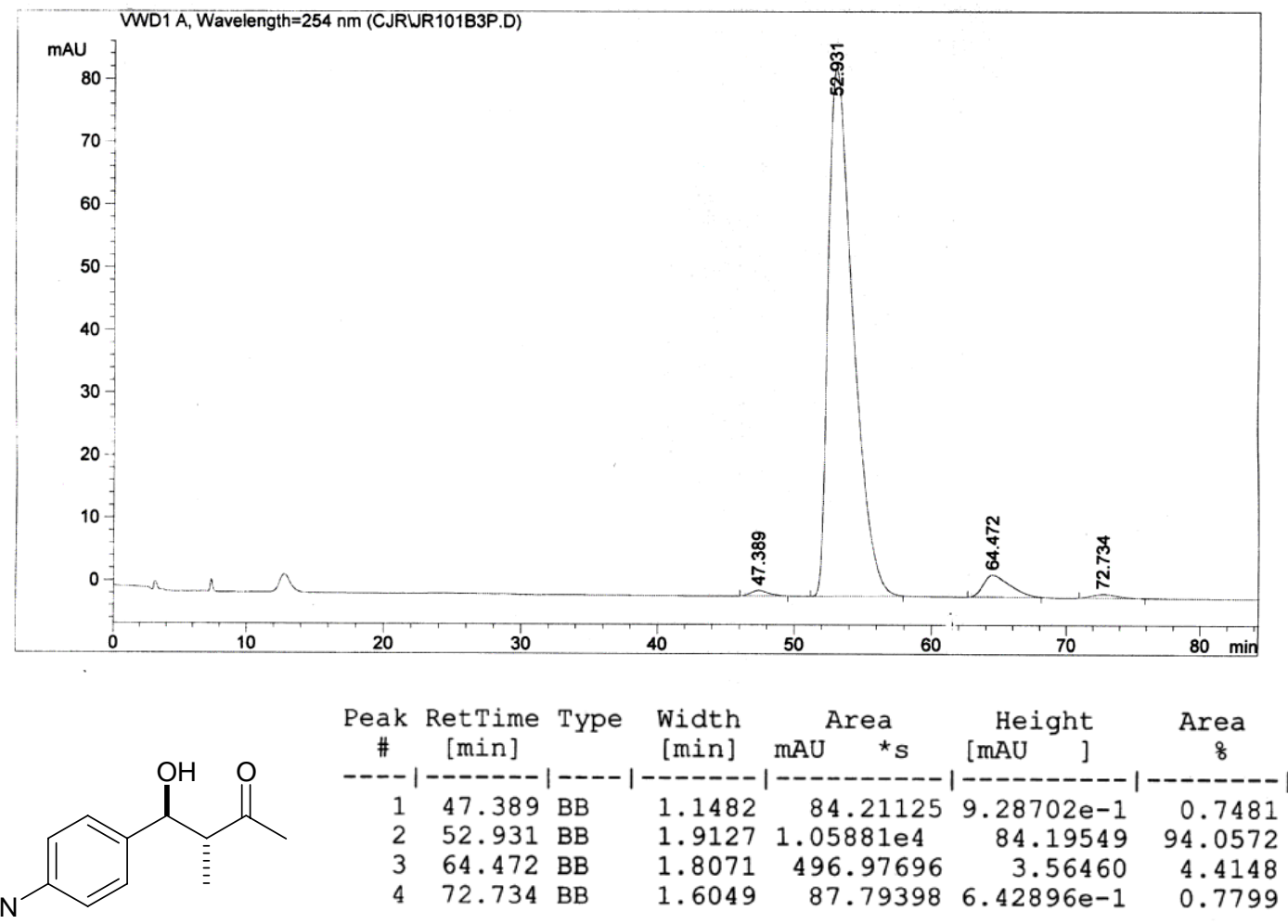

Totals :

$1.12571 \mathrm{e} 4 \quad 89.33168$ 
Crystal Structure of Catalyst 1a

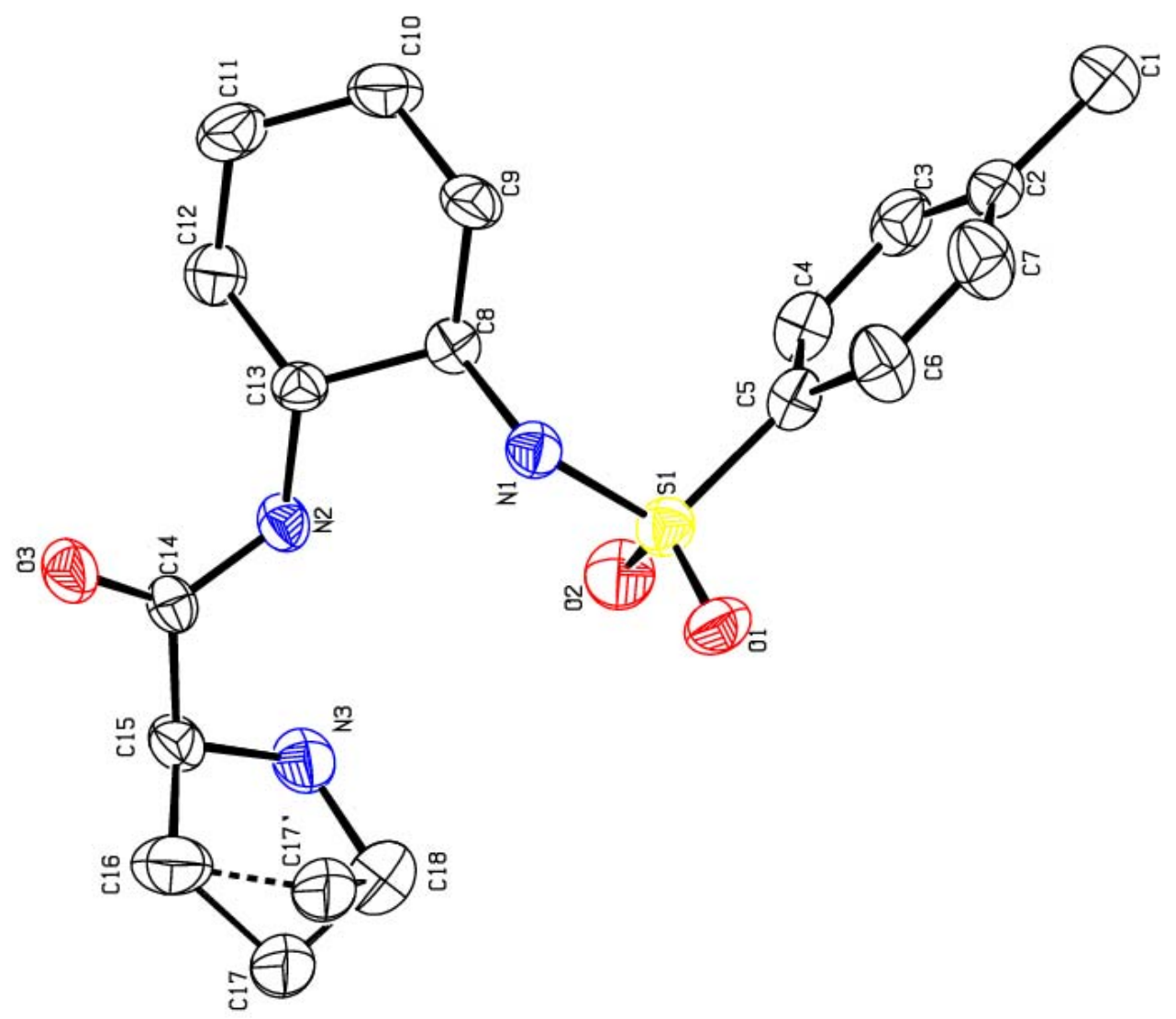




\section{Crystal Structure Data for Catalyst 1a}

data_t

_audit_creation_method

SHELXL-97

_chemical_name_systematic

;

?

;

_chemical_name_common

?

_chemical_melting_point

?

_chemical_formula_moiety

'C18 H27 N3 O3 S'

_chemical_formula_sum

'C18 H27 N3 O3 S'

_chemical_formula_weight

365.49

_chemical_absolute_configuration UNK

loop_

_atom_type_symbol

_atom_type_description

_atom_type_scat_dispersion_real

_atom_type_scat_dispersion_imag

_atom_type_scat_source

'C' 'C' $\mathbf{0 . 0 0 3 3} \mathbf{0 . 0 0 1 6}$

'International Tables Vol C Tables 4.2.6.8 and 6.1.1.4'

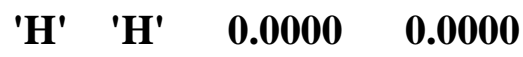

'International Tables Vol C Tables 4.2.6.8 and 6.1.1.4'

$\begin{array}{llll}\text { 'N' } & \text { 'N' } & 0.0061 & 0.0033\end{array}$

'International Tables Vol C Tables 4.2.6.8 and 6.1.1.4'

'O' 'O' $0.0106 \quad 0.0060$

'International Tables Vol C Tables 4.2.6.8 and 6.1.1.4'

'S' 'S' $0.1246 \quad 0.1234$

'International Tables Vol C Tables 4.2.6.8 and 6.1.1.4'

_symmetry_cell_setting Orthorhombic

_symmetry_space_group_name_H-M P2(1)2(1)2(1)

_symmetry_space_group_name_HalL 'P 2ac 2ab'

loop_

_symmetry_equiv_pos_as_xyz

'x, y, z'

'-x+1/2, $-y, z+1 / 2$ '

'-x, $y+1 / 2,-z+1 / 2$ '

'x+1/2, $-y+1 / 2,-z '$ 


\begin{tabular}{|c|c|}
\hline _cell_length_a & 9.8855(7) \\
\hline _cell_length_b & $9.8856(7)$ \\
\hline _cell_length_c & $20.3477(14)$ \\
\hline _cell_angle_alpha & 90.00 \\
\hline _cell_angle_beta & 90.00 \\
\hline _cell_angle_gamma & 90.00 \\
\hline _cell_volume & 1988.5(2) \\
\hline _cell_formula_units_Z & 4 \\
\hline _cell_measurement_temperature & 292(2) \\
\hline _cell_measurement_reflns_used & 1748 \\
\hline _cell_measurement_theta_min & 2.29 \\
\hline _cell_measurement_theta_max & 19.58 \\
\hline _exptl_crystal_description & block \\
\hline _exptl_crystal_colour & colorless \\
\hline _exptl_crystal_size_max & 0.24 \\
\hline _exptl_crystal_size_mid & 0.20 \\
\hline _exptl_crystal_size_min & 0.20 \\
\hline _exptl_crystal_density_meas & $?$ \\
\hline _exptl_crystal_density_diffrn & 1.221 \\
\hline _exptl_crystal_density_method & 'not measured' \\
\hline _exptl_crystal_F_000 & 784 \\
\hline _exptl_absorpt_coefficient_mu & 0.183 \\
\hline _exptl_absorpt_correction_type & multi-scan \\
\hline _exptl_absorpt_correction_T_min & $?$ \\
\hline _exptl_absorpt_correction_T_max & ? \\
\hline _exptl_absorpt_process_details & $?$ \\
\hline \multicolumn{2}{|l|}{ _exptl_special_details } \\
\hline \multicolumn{2}{|l|}{; } \\
\hline \multicolumn{2}{|l|}{; } \\
\hline _diffrn_ambient_temperature & 292(2) \\
\hline _diffrn_radiation_wavelength & 0.71073 \\
\hline _diffrn_radiation_type & MoKla \\
\hline _diffrn_radiation_source & 'fine-focus sealed tube' \\
\hline _diffrn_radiation_monochromator & graphite \\
\hline _diffrn_measurement_device_type & 'CCD area detector' \\
\hline _diffrn_measurement_method & 'phi and omega scans' \\
\hline _diffrn_detector_area_resol_mean & $?$ \\
\hline _diffrn_standards_number & ? \\
\hline _diffrn_standards_interval_count & $?$ \\
\hline
\end{tabular}




\begin{tabular}{ll} 
_diffrn_standards_interval_time & $?$ \\
_diffrn_standards_decay_\% & $?$ \\
_diffrn_reflns_number & 10678 \\
_diffrn_reflns_av_R_equivalents & 0.0856 \\
_diffrn_reflns_av_sigmaI/netI & 0.0818 \\
_diffrn_reflns_limit_h_min & -11 \\
_diffrn_reflns_limit_h_max & 10 \\
_diffrn_reflns_limit_k_min & -11 \\
_diffrn_reflns_limit_k_max & 11 \\
_diffrn_reflns_limit_l_min & -24 \\
_diffrn_reflns_limit_l_max & 23 \\
_diffrn_reflns_theta_min & 2.00 \\
_diffrn_reflns_theta_max & 25.00 \\
_reflns_number_total & 3506 \\
_reflns_number_gt & 2489 \\
_reflns_threshold_expression & $>2 s i g m a(I)$ \\
& \\
_computing_data_collection & 'Bruker SMART' \\
_computing_cell_refinement & 'Bruker SMART' \\
_computing_data_reduction & 'Bruker SAINT' \\
_computing_structure_solution & 'SHELXS-97 (Sheldrick, 1990)' \\
_computing_structure_refinement & 'SHELXL-97 (Sheldrick, 1997)' \\
_computing_molecular_graphics & 'Bruker SHELXTL' \\
_computing_publication_material & 'Bruker SHELXTL' \\
_refine_special_details & \\
; & \\
\hline &
\end{tabular}

Refinement of $F^{\wedge} 2^{\wedge}$ against ALL reflections. The weighted $R$-factor $w R$ and goodness of fit $S$ are based on $F \wedge 2^{\wedge}$, conventional $R$-factors $R$ are based on $F$, with $F$ set to zero for negative $F \wedge 2^{\wedge} \wedge$. The threshold expression of $F^{\wedge} 2^{\wedge}>2 \operatorname{sigma}\left(F^{\wedge} 2^{\wedge}\right)$ is used only for calculating $R$-factors(gt) etc. and is not relevant to the choice of reflections for refinement. R-factors based on $F^{\wedge} 2^{\wedge}$ are statistically about twice as large as those based on $F$, and $R$ factors based on ALL data will be even larger.

;

_refine_ls_structure_factor_coef Fsqd

_refine_ls_matrix_type full

_refine_ls_weighting_scheme_calc

_refine_ls_weighting_details

'calc $w=1 /\left[\backslash s^{\wedge} 2^{\wedge}\left(F_{0}{ }^{\wedge} 2^{\wedge}\right)+(0.0350 P)^{\wedge} 2^{\wedge}+0.0000 P\right]$ where $P=\left(F 0^{\wedge} 2^{\wedge}+2 F^{\wedge} c^{\wedge}{ }^{\wedge}\right) / 3^{\prime}$

_atom_sites_solution_primary_direct

_atom_sites_solution_secondary_difmap

_atom_sites_solution_hydrogens geom 


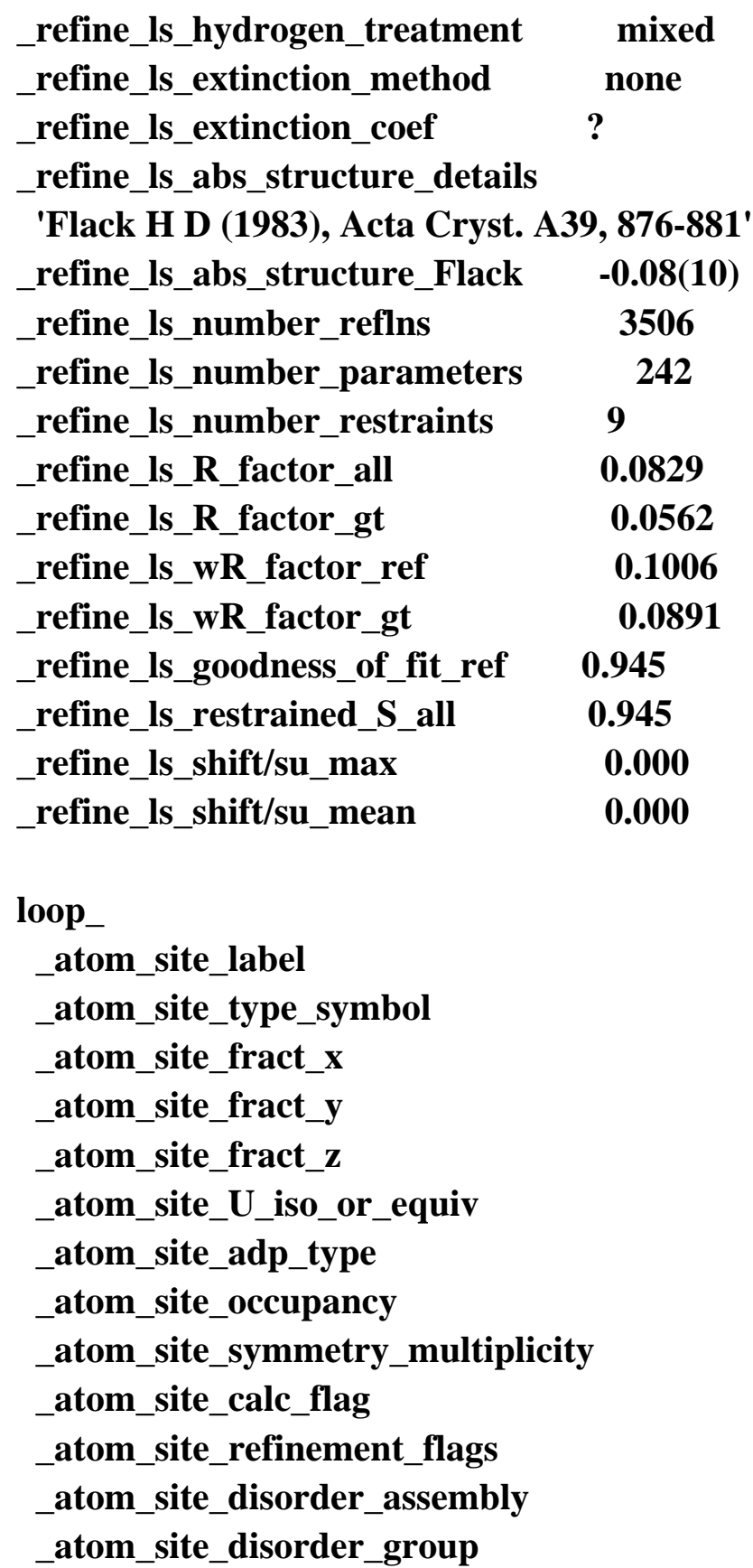

C1 C 0.2764(4) 0.6821(5) 0.36451(19) 0.0941(14) Uani 11 d ... H1A H 0.24130 .77260 .36570 .141 Uiso 11 calc $R$. . H1B H 0.3529 0.6754 0.3934 0.141 Uiso 11 calc R . . H1C H 0.2076 0.6199 0.3784 0.141 Uiso 11 calc R . .

C2 C 0.3193(4) 0.6484(4) 0.29582(18) 0.0642(10) Uani $11 \mathrm{~d}$. . . C3 C 0.4218(4) 0.5570(4) 0.28353(18) 0.0660(10) Uani $11 \mathrm{~d}$... H3 H 0.46770 .51890 .31890 .079 Uiso 11 calc $R$. .

C4 C 0.4585(3) 0.5201(3) 0.22073(18) 0.0597(9) Uani $11 \mathrm{~d}$. . . H4 H 0.52580 .45590 .21410 .072 Uiso 11 calc R . .

C5 C 0.3940(3) 0.5796(3) 0.16758(17) 0.0533(9) Uani $11 \mathrm{~d}$. . . C6 C 0.2961(4) 0.6759(4) 0.17824(19) 0.0725(11) Uani $11 \mathrm{~d}$... H6 H 0.2546 0.7188 0.1429 0.087 Uiso 11 calc R . . 
C7 C 0.2597(4) 0.7085(4) 0.2421(2) 0.0836(12) Uani 11 d . . H7 H 0.1927 0.7731 0.2489 0.100 Uiso 11 calc $R$. . C8 C 0.6864(3) 0.6570(3) 0.09824(15) 0.0457(8) Uani 11 d . . H8 H 0.6910 0.5794 0.1281 0.055 Uiso 11 calc $R$. . C9 C 0.7014(3) 0.7842(3) 0.13860(16) 0.0607(10) Uani $11 \mathrm{~d}$... H9A H 0.6321 0.7852 0.1723 0.073 Uiso 11 calc $R$. . H9B H 0.6869 0.8620 0.1105 0.073 Uiso 11 calc $R$. . C10 C 0.8391(4) 0.7965(4) 0.17102(18) 0.0744(11) Uani 11 d . . . H10A H 0.8454 0.8824 0.1938 0.089 Uiso 11 calc R . . H10B H 0.85030 .72470 .20300 .089 Uiso 11 calc R . . C11 C 0.9499(4) 0.7873(4) 0.11966(19) 0.0741(11) Uani 11 d ... H11A H 1.0377 0.7922 0.1408 0.089 Uiso 11 calc R . . H11B H 0.94250 .86250 .08930 .089 Uiso 11 calc R . . C12 C 0.9374(3) 0.6539(3) 0.08221(17) 0.0631(9) Uani $11 \mathrm{~d}$. . . H12A H 1.0086 0.6482 0.0495 0.076 Uiso 11 calc R . . H12B H 0.94830 .57890 .11250 .076 Uiso 11 calc R . . C13 C 0.7998(3) 0.6433(3) 0.04839(15) 0.0447(8) Uani $11 \mathrm{~d}$. . . H13 H 0.7922 0.7185 0.0172 0.054 Uiso 11 calc R . . C14 C 0.8428(3) 0.4959(3) -0.04649(15) 0.0455(8) Uani $11 \mathrm{~d}$... C15 C 0.8157(4) 0.3594(3) -0.07665(16) 0.0534(9) Uani 11 d . B . H15 H 0.9025 0.3151 -0.0855 0.064 Uiso 11 calc R . . C16 C 0.7380(5) 0.3716(4) -0.14104(19) 0.0876(13) Uani 11 d D . . H16A H 0.7002 0.4617 -0.1456 0.105 Uiso 0.80 1 calc PR A 1 H16B H 0.7973 0.3544 -0.1781 0.105 Uiso 0.80 1 calc PR A 1 H16C H 0.7513 0.4614 -0.1612 0.105 Uiso 0.20 1 d PR A 2 H16D H 0.7605 0.3029 -0.1718 0.105 Uiso 0.20 1 d PR A 2 C17' C 0.5984(12) 0.3564(19) -0.1159(10) 0.118(3) Uani 0.20 1 d PD B 2 H17D H 0.5364 0.3334 -0.1510 0.141 Uiso 0.20 1 calc PR B 2 H17C H 0.5678 0.4390 -0.0949 0.141 Uiso 0.20 1 calc PR B 2 C17 C 0.6275(6) 0.2683(8) -0.1385(2) 0.118(3) Uani 0.80 1 d PD B 1 H17B H 0.5452 0.3028 -0.1582 0.141 Uiso 0.80 1 calc PR B 1 H17A H 0.6541 0.1860 -0.1610 0.141 Uiso 0.80 1 calc PR B 1 C18 C 0.6089(5) 0.2439(6) -0.0673(2) 0.1091(16) Uani 11 d D . . H18A H 0.5383 0.3023 -0.0502 0.131 Uiso 0.80 1 calc PR B 1 H18B H 0.5823 0.1507 -0.0598 0.131 Uiso 0.80 1 calc PR B 1 H18C H 0.5327 0.2500 -0.0354 0.131 Uiso 0.20 1 d PR B 2 H18D H 0.6076 0.1593 -0.0879 0.131 Uiso 0.20 1 d PR B 2 N1 N 0.5526(3) 0.6551(3) 0.06538(12) 0.0501(7) Uani $11 \mathrm{~d}$ D . . N2 N 0.7870(3) 0.5183(3) 0.01187(13) 0.0487(7) Uani $11 \mathrm{~d}$ D . . N3 N 0.7354(4) 0.2718(3) -0.03412(16) 0.0646(8) Uani 11 d D B . O1 O 0.3284(2) 0.5597(2) 0.04482(11) 0.0671(7) Uani $11 \mathrm{~d}$... $\mathrm{O} 2 \mathrm{O} 0.5118(2)$ 0.4153(2) 0.08754(13) 0.0731(7) Uani $11 \mathrm{~d}$... O3 O 0.9126(2) 0.5808(2) -0.07526(11) 0.0663(7) Uani $11 \mathrm{~d}$... S1 S 0.44327(9) 0.54259(8) 0.08667(4) 0.0544(2) Uani 11 d . . . 
HЗА H 0.779(3) 0.198(2) -0.0283(17) 0.068(13) Uiso $11 \mathrm{~d}$ D . . H1 H 0.517(3) 0.7322(16) 0.0598(13) 0.044(10) Uiso $11 \mathrm{~d}$ D . . H2 H 0.740(3) 0.451(2) 0.0259(13) 0.046(10) Uiso $11 \mathrm{~d}$ D . .

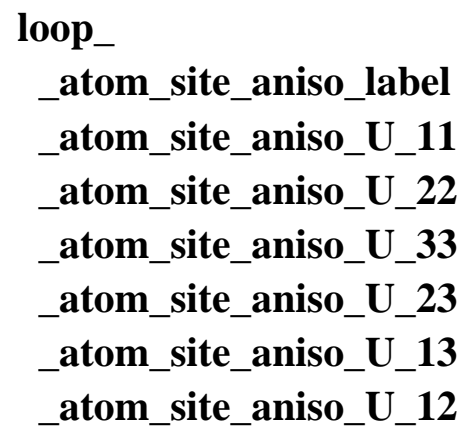

All esds (except the esd in the dihedral angle between two l.s. planes) are estimated using the full covariance matrix. The cell esds are taken into account individually in the estimation of esds in distances, angles 
and torsion angles; correlations between esds in cell parameters are only used when they are defined by crystal symmetry. An approximate (isotropic) treatment of cell esds is used for estimating esds involving l.s. planes.

;

loop_

_geom_bond_atom_site_label_1

_geom_bond_atom_site_label_2

_geom_bond_distance

_geom_bond_site_symmetry_2

_geom_bond_publ_flag

C1 C2 1.498(5).?

C1 H1A 0.9600 .?

C1 H1B 0.9600 . ?

C1 H1C 0.9600 .?

C2 C7 1.377(5) . ?

C2 C3 1.381(5). ?

C3 C4 1.377(5) . ?

С3 H3 0.9300 . ?

C4 C5 1.386(4). ?

C4 H4 0.9300 . ?

C5 C6 1.375(4). ?

C5 S1 1.755(3) . ?

C6 C7 1.386(5). ?

C6 H6 0.9300.?

C7 H7 0.9300 . ?

C8 N1 1.482(4). ?

C8 C9 1.509(4) . ?

C8 C13 1.518(4) . ?

C8 H8 0.9800 . ?

C9 C10 1.518(4) . ?

C9 H9A 0.9700 . ?

C9 H9B 0.9700 . ?

C10 C11 1.517(5) . ?

C10 H10A 0.9700 . ?

C10 H10B 0.9700 . ?

C11 C12 1.528(5) . ?

C11 H11A 0.9700 . ?

C11 H11B 0.9700 . ?

C12 C13 1.528(4) . ?

C12 H12A 0.9700 . ?

C12 H12B 0.9700 . ?

C13 N2 1.448(4) . ?

C13 H13 0.9800 . ? 


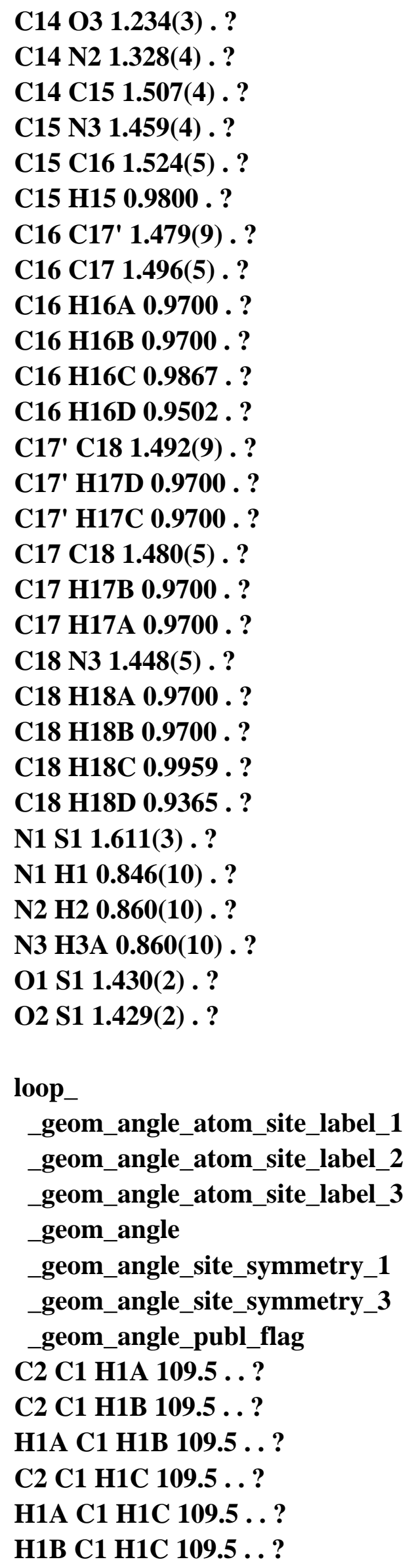


C7 C2 C3 116.9(4) .. ?

C7 C2 C1 121.6(4) .. ?

C3 C2 C1 121.5(4) .. ?

C4 C3 C2 122.3(4) .. ?

C4 C3 H3 118.9 . . ?

C2 C3 H3 118.9 .. ?

C3 C4 C5 119.4(3) .. ?

C3 C4 H4 120.3 . . ?

C5 C4 H4 120.3 .. ?

C6 C5 C4 119.6(3) . . ?

C6 C5 S1 119.2(3) .. ?

C4 C5 S1 121.1(3) .. ?

C5 C6 C7 119.5(3) .. ?

C5 C6 H6 120.3 .. ?

C7 C6 H6 120.3 .. ?

C2 C7 C6 122.2(4) .. ?

C2 C7 H7 118.9 .. ?

C6 C7 H7 118.9 .. ?

N1 C8 C9 110.1(3) .. ?

N1 C8 C13 110.9(2) .. ?

C9 C8 C13 111.4(3) .. ?

N1 C8 H8 108.1 .. ?

C9 C8 H8 108.1 .. ?

C13 C8 H8 108.1 .. ?

C8 C9 C10 113.0(3) .. ?

C8 C9 H9A 109.0 . . ?

C10 C9 H9A 109.0 .. ?

C8 C9 H9B 109.0 . . ?

C10 C9 H9B 109.0 .. ?

H9A C9 H9B 107.8 . . ?

C11 C10 C9 110.1(3) . . ?

C11 C10 H10A 109.6 .. ?

C9 C10 H10A 109.6 .. ?

C11 C10 H10B 109.6 .. ?

C9 C10 H10B 109.6 .. ?

H10A C10 H10B 108.2 .. ?

C10 C11 C12 109.7(3) . . ?

C10 C11 H11A 109.7 . . ?

C12 C11 H11A 109.7 . . ?

C10 C11 H11B 109.7 .. ?

C12 C11 H11B 109.7 .. ?

H11A C11 H11B 108.2 .. ?

C13 C12 C11 110.9(3) . . ?

C13 C12 H12A 109.5 . . ? 
C11 C12 H12A 109.5 . . ?

C13 C12 H12B 109.5 .. ?

C11 C12 H12B 109.5 .. ?

H12A C12 H12B 108.1 .. ?

N2 C13 C8 110.8(3) .. ?

N2 C13 C12 111.5(3) . . ?

C8 C13 C12 110.5(2) . . ?

N2 C13 H13 108.0 .. ?

C8 C13 H13 108.0 .. ?

C12 C13 H13 108.0 .. ?

O3 C14 N2 122.9(3) . . ?

O3 C14 C15 121.0(3) . . ?

N2 C14 C15 116.1(3) . . ?

N3 C15 C14 112.8(3) . . ?

N3 C15 C16 106.4(3) . . ?

C14 C15 C16 111.7(3) . . ?

N3 C15 H15 108.6 . . ?

C14 C15 H15 108.6 . . ?

C16 C15 H15 108.6 . . ?

C17' C16 C15 99.5(8) . . ?

C17 C16 C15 106.5(4) . . ?

C17' C16 H16A 76.6 . . ?

C17 C16 H16A 110.4 . . ?

C15 C16 H16A 110.4 .. ?

C17' C16 H16B 144.6 . . ?

C17 C16 H16B 110.4 .. ?

C15 C16 H16B 110.4 .. ?

H16A C16 H16B 108.6 .. ?

C17' C16 H16C 111.1 .. ?

C17 C16 H16C 136.6 . . ?

C15 C16 H16C 111.2 .. ?

H16B C16 H16C 75.8 . . ?

C17' C16 H16D 112.0 . . ?

C17 C16 H16D 72.9 .. ?

C15 C16 H16D 113.0 .. ?

H16A C16 H16D 133.1 .. ?

H16C C16 H16D 109.8 . . ?

C16 C17' C18 103.9(8) . . ?

C16 C17' H17D 111.0 .. ?

C18 C17' H17D 111.0 .. ?

C16 C17' H17C 111.0 .. ?

C18 C17' H17C 111.0 .. ?

H17D C17' H17C 109.0 . . ?

C18 C17 C16 103.7(4) . . ? 
C18 C17 H16D 125.6 . . ?

C18 C17 H17B 111.0 .. ?

C16 C17 H17B 111.0 .. ?

H16D C17 H17B 117.6 .. ?

C18 C17 H17A 111.0 .. ?

C16 C17 H17A 111.0 . . ?

H16D C17 H17A 75.1 . . ?

H17B C17 H17A 109.0 .. ?

C16 C17 H18D 127.5 . . ?

H16D C17 H18D 125.5 .. ?

H17B C17 H18D 115.2 . . ?

H17A C17 H18D 75.9 .. ?

N3 C18 C17 108.5(4) . . ?

N3 C18 C17' 103.1(8) . . ?

N3 C18 H18A 110.0 . . ?

C17 C18 H18A 110.0 . . ?

C17' C18 H18A 75.1 .. ?

N3 C18 H18B 110.0 .. ?

C17 C18 H18B 110.0 . . ?

C17' C18 H18B 142.5 . . ?

H18A C18 H18B 108.4 .. ?

N3 C18 H18C 109.8 . . ?

C17 C18 H18C 136.2 .. ?

C17' C18 H18C 109.5 .. ?

H18B C18 H18C 75.6 . . ?

N3 C18 H18D 113.0 . . ?

C17 C18 H18D 73.0 .. ?

C17' C18 H18D 111.6 .. ?

H18A C18 H18D 133.1 .. ?

H18C C18 H18D 109.6 . . ?

C8 N1 S1 119.1(2) . . ?

C8 N1 H1 115(2) . . ?

S1 N1 H1 112(2) . . ?

C14 N2 C13 124.4(3) .. ?

C14 N2 H2 112.9(19) . . ?

C13 N2 H2 122.7(19) .. ?

C18 N3 C15 107.9(3) . . ?

C18 N3 H3A 110(2) . . ?

C15 N3 H3A 108(2) . . ?

O2 S1 01 119.23(16) . . ?

O2 S1 N1 107.05(15) . . ?

O1 S1 N1 106.94(15) . . ?

O2 S1 C5 107.66(16) . . ?

O1 S1 C5 108.28(15) .. ? 
N1 S1 C5 107.12(14) . . ?

loop_

_geom_torsion_atom_site_label_1

_geom_torsion_atom_site_label_2

_geom_torsion_atom_site_label_3

_geom_torsion_atom_site_label_4

_geom_torsion

_geom_torsion_site_symmetry_1

_geom_torsion_site_symmetry_2

_geom_torsion_site_symmetry_3

_geom_torsion_site_symmetry_4

_geom_torsion_publ_flag

C7 C2 C3 C4 -3.9(5) .... ?

C1 C2 C3 C4 177.0(3) .... ?

C2 C3 C4 C5 2.2(5) .... ?

C3 C4 C5 C6 1.0(5) .... ?

C3 C4 C5 S1 176.6(3) .... ?

C4 C5 C6 C7 -2.4(5) .... ?

S1 C5 C6 C7 -178.1(3) .... ?

C3 C2 C7 C6 2.4(6) .... ?

C1 C2 C7 C6 -178.4(4) .... ?

C5 C6 C7 C2 0.6(6) .... ?

N1 C8 C9 C10 -177.1(3) .... ?

C13 C8 C9 C10 -53.6(4) .... ?

C8 C9 C10 C11 55.5(4) .... . ?

C9 C10 C11 C12 -57.5(4) .... ?

C10 C11 C12 C13 59.3(4) .... ?

N1 C8 C13 N2 -59.3(3) ... . ?

C9 C8 C13 N2 177.7(3) .... ?

N1 C8 C13 C12 176.6(2) .... ?

C9 C8 C13 C12 53.6(3) .... . ?

C11 C12 C13 N2 179.2(3) ... . ?

C11 C12 C13 C8 -57.1(4) ... . ?

O3 C14 C15 N3 177.9(3) ... . ?

N2 C14 C15 N3 -2.3(4) .... ?

O3 C14 C15 C16 -62.3(4) .... ?

N2 C14 C15 C16 117.4(3) .... ?

N3 C15 C16 C17' 30.0(9) .... ?

C14 C15 C16 C17' -93.4(9) .... ?

N3 C15 C16 C17 -10.9(5) .... ?

C14 C15 C16 C17 -134.3(4) ... . ?

C17 C16 C17' C18 61.7(8) .... ?

C15 C16 C17' C18 -42.7(15) .... ? 
C17' C16 C17 C18 -62.4(8) ... . ?

C15 C16 C17 C18 22.8(6) ... . ?

C16 C17 C18 N3 -27.2(7) .... ?

C16 C17 C18 C17' 61.4(8) .... ?

C16 C17' C18 N3 40.6(16) ... . ?

C16 C17' C18 C17 -62.7(8) .... ?

C9 C8 N1 S1 -113.5(3) ... . ?

C13 C8 N1 S1 122.7(2) .... ?

O3 C14 N2 C13 0.5(5) .... ?

C15 C14 N2 C13 -179.2(3) ... . ?

C8 C13 N2 C14 158.7(3) .... ?

C12 C13 N2 C14 -77.8(4) .... ?

C17 C18 N3 C15 21.0(6) .... ?

C17' C18 N3 C15 -20.7(9) .... ?

C14 C15 N3 C18 116.8(3) ... . ?

C16 C15 N3 C18 -5.9(4) .... ?

C8 N1 S1 O2 -47.9(3) ....?

C8 N1 S1 01 -176.7(2) ... . ?

C8 N1 S1 C5 67.4(3) .... ?

C6 C5 S1 O2 -162.5(3) .... ?

C4 C5 S1 O2 21.9(3) ... . ?

C6 C5 S1 01 -32.4(3) .... ?

C4 C5 S1 O1 152.0(3) ... . ?

C6 C5 S1 N1 82.7(3) ... . ?

C4 C5 S1 N1 -93.0(3) .... ?

loop_

_geom_hbond_atom_site_label_D

_geom_hbond_atom_site_label_H

_geom_hbond_atom_site_label_A

_geom_hbond_distance_DH

_geom_hbond_distance_HA

_geom_hbond_distance_DA

_geom_hbond_angle_DHA

_geom_hbond_site_symmetry_A

C15 H15 O2 0.982 .52 3.344(4) 141.54

C13 H13 O1 0.982 .55 3.506(4) 163.7 4_565

N1 H1 O3 0.846(10) 2.141(13) 2.961(4) 163(3) 4_465

N2 H2 N3 0.860(10) 2.15(3) 2.660(4) 118(2) .

$\mathrm{N} 2 \mathrm{H} 2 \mathrm{O} 2 \quad 0.860(10) 2.61(2) 3.288(4)$ 137(2) .

N3 H3A O1 $0.860(10) 2.612(18) 3.411(4)$ 155(3) 4

_diffrn_measured_fraction_theta_max $\mathbf{1 . 0 0 0}$

_diffrn_reflns_theta_full $\quad 25.00$ 
_diffrn_measured_fraction_theta_full 1.000

_refine_diff_density_max 0.148

_refine_diff_density_min $\quad-0.209$

_refine_diff_density_rms $\quad 0.039$ 